\title{
Quantum interface between light and atomic ensembles
}

\author{
Klemens Hammerer \\ Institute for Theoretical Physics, Technikerstr. 25, 6020 Innsbruck, Austria
}

Anders S. Sørensen and Eugene S. Polzik

Niels Bohr Institute, Copenhagen University, Blegdamsvej 17, Copenhagen 2100, Denmark

During the past decade the interaction of light with multi-atom ensembles has attracted a lot of attention as a basic building block for quantum information processing and quantum state engineering. The field started with the realization that optically thick free space ensembles can be efficiently interfaced with quantum optical fields. By now the atomic ensemble - light interfaces have become a powerful alternative to the cavity-enhanced interaction of light with single atoms. We discuss various mechanisms used for the quantum interface, including quantum nondemolition or Faraday interaction, quantum measurement and feedback, Raman interaction and electromagnetically induced transparency. The paper provides a common theoretical frame for these processes, describes basic experimental techniques and media used for quantum interfaces, and reviews several key experiments on quantum memory for light, quantum entanglement between atomic ensembles and light, and quantum teleportation with atomic ensembles. We concentrate on deterministic operations but also briefly discuss probabilistic protocols. The paper concludes with an outlook on the future of atomic ensembles as an enabling technology in quantum information processing.

\section{Contents}

I. Introduction

A. History and motivation

B. Elementary level schemes

II. Theoretical Background

A. Description of light and atoms

B. Interaction of light with model atoms

C. Theory Including Spontaneous emission

D. Realistic Multilevel Atoms

E. Ensemble in Magnetic Field

F. Quantum measurement and feedback

G. Other Strategies

H. Summary of the theory

III. Atomic media for quantum interface
A. Room temperature gases
B. Cold and trapped atoms
C. Solid state
D. Other possible media

IV. Entanglement of Atomic Ensembles

A. Spin-Squeezing in a Single Ensemble

B. Deterministic entanglement

C. Probabilistic entanglement

V. Quantum Memory for Light
A. Figure of Merit
B. QND \& Feedback Protocol
C. Multipass Approaches
D. Raman and EIT approach
E. Photon Echo

VI. Quantum teleportation between light and atoms 37 A. Quantum Teleportation

B. Teleportation based on Faraday interaction

VII. Errors and fidelity for different interfaces

VIII. Outlook

Acknowledgments
IX. Appendices

A. Adiabatic Elimination
B. Three dimensional Hamiltonians
C. Propagation equations for light
D. Inclusion of spontaneous emission
E. Dimensionless equations of motion
F. Tensor Decomposition

References

\section{INTRODUCTION}

\section{A. History and motivation}

Quantum features of atom-light interaction have been among the central issues in physics since the early days of quantum mechanics. Starting in the $60 \mathrm{~s}$, with the development of quantum optics - the field where second quantization of light is central - quantum electrodynamics became part of optical and atomic physics. For decades after that the inherently quantum features of atom-light interaction have been studied primarily within the framework of cavity Quantum Electrodynamics (QED) where light can be efficiently coupled to a few atoms or even to a single atom. Despite the spectacular progress achieved in this direction, the complexity and technical challenges associated with an atom strongly coupled to a high-finesse cavity were calling for alternative approaches.

A new approach to the matter-light quantum interface came with the realization of the fact that a large collection of atoms - an atomic ensemble - can be efficiently coupled to quantum light if a collective superposition state of many atoms can be utilized for the coupling. The simplest example of such coupling is presented in Fig. 17. A collection of atoms in the ground state is illuminated with two modes of light $a_{+}$and $a_{-}$. As shown 
by Kuzmich et al. (1997), if the modes possess quantum correlations (entanglement) and light is absorbed by the atomic ensemble, the quantum correlations can be mapped on the collective superposition of the two final states of atoms. The strong coupling condition in this case amounts to the requirement of the large resonant optical depth $d$ of the atomic ensemble. It later turned out, that the requirement $d \gg 1$ is the most significant requirement for all types of the quantum interface between atomic ensembles and light known up to now. The experiment demonstrating that a quantum feature of radiation (squeezing) can be transferred onto atoms via the process shown in Fig. 19a was performed by Hald et al. (1999). This approach has been further developed using photon echo ideas (Moiseev, 2003).

Quantum nondemolition (QND) measurement (Braginsky and Khalili, 1996) on radiation interacting with atoms has emerged as a powerful tool for quantum state engineering, first in the cavity QED setting and then in the atomic ensemble context as an efficient method for generation of spin squeezing (Kuzmich et al. , 1998). Shortly thereafter QND with atomic ensembles has become one of the main instruments for the quantum interface.

Simultaneously with these developments yet another approach was developed based on Electromagnetically Induced Transparency (EIT) (Boller et al., 1991; Fleischhauer et al. 2005$)$. After Hau et al. (1999) demonstrated that EIT allows for very slow propagation of light through an atomic ensemble it was quickly realized that reducing the group velocity to zero would enable an atomic memory for light (Fleischhauer and Lukin, 2000 Lukin et al., 2000) and the first experimental demonstrations of this for classical pulses have been presented in Liu et al. (2001); Phillips et al. (2001).

The process depicted in Fig. 17a is a rudimentary example of one of the main routines for atoms-light quantum interface: the quantum state transfer from light to atoms, or the quantum memory for light. The ability for mapping, storing, and retrieving quantum states of light - the natural long-distance carrier of information - onto the material storage medium is one of the major enabling procedures in quantum information processing. In this review we cover various approaches to the quantum memory, including the Raman process, EIT, photon echo, and the QND measurement and feedback. We review methods which provide a long term quantum memory with the fidelity better than any classical procedure can achieve. The interface can be implemented either via interaction only or by the teleportation-like procedure involving generation of entanglement, Bell measurement on light and quantum feedback onto atoms. In this article we will discuss both approaches.

The quantum interface can be formulated either in the Schrödinger or in the Heisenberg picture. For example, the transformation of a quantum state of light into a quantum state of atoms in the Schrödinger picture $\hat{U}\left|\Psi_{L} 0_{A}\right\rangle \rightarrow\left|0_{L} \Psi_{A}\right\rangle$ corresponds to the operator trans- formation $\hat{U}^{\dagger} \hat{a}_{A} \hat{U}=a_{L}$ in the Heisenberg picture. The two pictures are equivalent, and we will mostly use the Heisenberg picture throughout this review.

The second most important routine for the quantum interface is generation of entanglement between light and atoms. The light/atoms entanglement in turn enables generation of entanglement between remote atomic ensembles, as well as atomic teleportation and entanglement swapping protocols. Furthermore the light-atom entanglement also allows for quantum memory through light-atom teleportation.

In this review we concentrate on deterministic entanglement generation and detection. Probabilistic protocols based on generation of a single collective atomic excitation of an atomic ensemble following detection of a photon emitted by the ensemble have been actively developed in the recent years (Chaneliere et al., 2007; Chou et al., 2004, 2007, Kuzmich et al., 2003: Matsukevich and Kuzmich, 2004; van der Wal et al., 2003). This approach which has been triggered by a proposal for a quantum repeater with atomic ensembles (Duan et al. 2001 ), has been also highly successful in generation of single photons on demand. It deserves a separate review paper and will be only briefly discussed in this article.

The requirements for the atomic memory may differ depending on the particular application. A distributed quantum computer network requires the complete set of memory capabilities: mapping of the light state onto memory, storage and operations on the memory state, and retrieval of the memory state back onto light for further processing. Applications in quantum communications, which involve local operations on stored states and classical communications between partners, often only require a measurement of the memory state in a specific basis, i.e., no full retrieval of the quantum state of the memory back onto light is necessary. Yet other proposals, such as linear optics quantum computing which uses off-line entanglement resources require only the retrieval of the atomic state onto light but it has to be efficient and deterministic (Menicucci et al. 2006).

\section{B. Elementary level schemes}

Naturally, the atomic levels used for storage of quantum state should be long lived, with particular requirements for the lifetime depending on applications. For example, for a memory used in a long distance communication protocol, the memory lifetime usually should be longer than the time required for classical communication over this distance. For a few hundred kilometers this time is of the order of $10^{-3} \mathrm{sec}$. Short distance applications may require shorter memory time but one should keep in mind that a low loss fiber loop can be a strong competitor for a short term atomic quantum memory for light (Pittman and Franson, 2002). A $5 \mathrm{~km}$ fiber loop can, in principle, store a photon for $25 \mu \mathrm{sec}$ with only $20 \%$ losses at the telecom wavelength. However even for short stor- 
age times atoms may have an advantage over the fiber loop if a nontrivial operation has to be performed on the stored quantum states.

Optical atomic transitions used for coupling light to the storage ground states of atoms should be strong in order to have a large bandwidth of the memory. Therefore strong dipole allowed transitions are typically used for the interaction. Figs. 1(b), (c), and (d) present the atomic level schemes typically used for the interface. The (b) and (c) parts of the figure present the $\Lambda$ - scheme used in the Raman and EIT memory schemes as well for entanglement generation. The (d) part shows the so-called Faraday interaction which is sometimes also referred to as QND interaction for reasons discussed below.

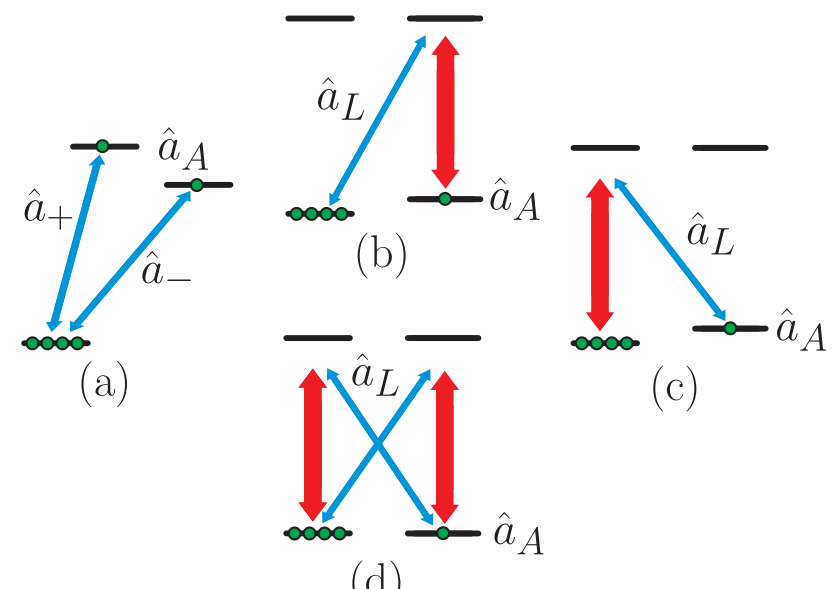

(d)

FIG. 1 Elementary level schemes: (a) A simple absorption scheme with the quantum fields $a_{ \pm}$mapped onto the atomic states $a_{A}$, (b) Beam splitter type interaction - basis for Raman and EIT memory schemes, (c) Parametric gain type interaction - basis for entanglement schemes, (d) Quantum nondemolition (or Faraday) interaction - basis for entanglement, memory and teleportation schemes

In (b) the atoms are prepared in the ground state coupled to the quantum field $\hat{a}_{L}$ (thin line), with the bold line showing a strong coupling field. The interaction Hamiltonian for such a system, after adiabatic elimination of the excited state, can be cast in the form $\hat{H} \sim \hat{a}_{L} \hat{a}_{A}^{\dagger}+$ H.C. In quantum optics this Hamiltonian is often referred to as the beam-splitter Hamiltonian. The interface mixes the input atom and light states as a "beamsplitter" and the "reflection coefficient" of unity corresponds to a perfect state swapping between light and atoms. The detailed derivation of this Hamiltonian for the light-atoms interaction will be given later in the article, but the intuitive picture is obvious - if a single photonic excitation is removed (annihilated) from the field $\hat{a}_{L}$, a single collective atomic excitation $\hat{a}_{A}$ is created. If this process can be run efficiently, it works as Ramantype quantum memory for light introduced for atomic ensembles by Kozhekin et al. (2000) and described in Sec. V.D The same level scheme can be used for the EIT-based memory (Fleischhauer and Lukin, 2000, Lukin et al. 2000), although the Hamiltonian-based approach is not quite applicable in this case (see the detailed theory in Secs. II and V.D. EIT based quantum memory experiments are described in Sec. V.D.

Part (c) of the figure shows the same atomic structure but now the fields are arranged in a way which can be used for the atoms-light entangling interaction with the Hamiltonian $\hat{H} \sim \hat{a}_{L} \hat{a}_{A}+$ H.C. The Hamiltonian is formally identical to the parametric gain interaction Hamiltonian which has been a workhorse for studies of entangled and non-classical states of light since the 60 s. The important new feature in the present case is that the two entangled operators belong to a light mode and an atomic mode respectively. This kind of entanglement has been used for unconditional light-to-atoms teleportation experiment described in Sec. VI and its probabilistic version discussed in Sec. IV.Clis the basis for the repeater protocol of Duan et al. (2001).

To complete the discussion of elementary level schemes used for basic interface routines we consider the four-level scheme shown in figure Fig. 1(d). Obviously the Hamiltonian for this interaction can be obtained by combining the beam-splitter Hamiltonian (Fig. 1 (b)) and the parametric entangling Hamiltonian (Fig. 1 (c)) provided that the two quantum fields (thin lines) belong to the same mode: $\hat{H} \sim \hat{P}_{L} \hat{P}_{A}$ where the canonical operators for light and atoms which obey the canonical commutation relation $[\hat{X}, \hat{P}]=i$ have been introduced. This interaction allows for a QND measurement of the atomic operator $P_{A}$ by means of detection of the light operator $X_{L}$. As discussed in detail in Sections IV A,B the QND measurement projects atoms into an entangled state. The same interaction is often called the quantum Faraday interaction because in case of magnetic levels it leads to polarization rotation of light. The QND-Faraday interaction of atoms and light followed by the measurement on the light and the feedback conditioned on the measurement applied onto atoms was used to demonstrate quantum memory for light as described in Section $\mathrm{V}$.

To summarize, the basic features of most quantum interface protocols to date can be understood by analyzing simple 3- or 4-level atoms. Besides the condition $d \gg 1$ mentioned above, another unifying feature for all approaches which use multi-atom ensembles is the possibility to initialize the ensemble, e.g., by optical pumping, in one of the ground substates. Choosing the polarizations and frequencies of the quantum and classical coupling fields one can choose between various routines, such as memory, entanglement and Faraday interaction, as shown in Fig. 1. In the following theoretical description we will provide a unified approach to all these types of interfaces.

In the literature the interface protocols are often divided into those for states of continuous variables and those for discrete variables, or qubits. The former are usually based on the Faraday interaction and are described most conveniently in terms of $X, P$ operators measured by homodyne detection, while the latter are 
commonly based on the $\Lambda$-type interactions in combination with counting of single photons and are most easily described in terms of $a, a^{\dagger}$ representations. One of the goals of this review is to show that continuous and discrete variable protocols can in many aspects be treated on equal footing, and that the choice of variables is defined by the convenience of description and the type of measurements involved. E.g., in the ideal limit a memory protocol which is most conveniently described by $X, P$ operators could be used to store a single photon with perfect fidelity. On the other hand, the state of atomic memory in protocols which use $a, a^{\dagger}$ representation can be conveniently analyzed by atomic tomography in the $X, P$ basis. The type of errors which appear under nonideal conditions is of course different for different protocols, but irrespectively of the specific protocol, the condition $d \gg 1$, leads to fewer errors. Which protocol to use, for a given optical density, therefore depends on the specific application and a detailed analysis of the imperfections should be made in each situation.

This review strives to provide a coherent picture of the work on the quantum interface between light and atomic ensembles using various approaches, atomic media, and protocols. It includes the discussion of major experimental achievements to date and concludes with the analysis of the current limitations and future goals.

\section{THEORETICAL BACKGROUND}

\section{A. Description of light and atoms}

Harmonic oscillators Throughout this review we shall be dealing with single modes of the electromagnetic field and collective spin excitations of atomic ensembles which can be well approximated by harmonic oscillators with canonical position and momentum operators $X_{n}$ and $P_{n}$, where $n$ refers to the mode number. In most cases we shall omit the hats on the operators in what follows. These canonical operators are dimensionless with the standard commutator

$$
\left[X_{n}, P_{m}\right]=i \delta_{m n}
$$

Instead of the $X_{n}$ and $P_{n}$ operators the harmonic oscillators can also be described in terms of the annihilation operators

$$
a_{n}=\frac{1}{\sqrt{2}}\left(X_{n}+i P_{n}\right)
$$

which have commutation relation $\left[a_{n}, a_{m}^{\dagger}\right]=\delta_{n m}$.

Instead of labeling by a discrete number $n$ the modes can be denoted by a continuous parameter, e.g., the position vector $\vec{r}$ with the commutation relations

$$
\begin{aligned}
& {\left[X(\vec{r}), P\left(\vec{r}^{\prime}\right)\right]=i \delta\left(\vec{r}-\vec{r}^{\prime}\right),} \\
& {\left[a(\vec{r}), a^{\dagger}\left(\vec{r}^{\prime}\right)\right]=\delta\left(\vec{r}-\vec{r}^{\prime}\right) .}
\end{aligned}
$$

In some cases we deal with the storage or transfer of a set of $n$ modes. Such discrete modes can be constructed from the continuous modes by introducing a complete orthogonal set of mode functions $\left\{u_{m}(\vec{r})\right\}$ satisfying

$$
\begin{aligned}
\int d \vec{r} u_{m}^{*}(\vec{r}) u_{n}(\vec{r}) & =\delta_{m n} \\
\sum_{m} u_{m}^{*}(\vec{r}) u_{m}\left(\vec{r}^{\prime}\right) & =\delta\left(\vec{r}-\vec{r}^{\prime}\right) .
\end{aligned}
$$

If we now define the discrete annihilation operators

$$
a_{m}=\int d \vec{r} u_{m}^{*}(\vec{r}) a(\vec{r})
$$

they have the appropriate commutator $\left[a_{n}, a_{m}^{\dagger}\right]=\delta_{m n}$.

Light Light beams travelling in the $z$-direction can be described (in c.g.s. units) in the paraxial approximation by a quantized electric field

$$
\vec{E}(\vec{r})=\sum_{m, \sigma, k} \sqrt{\frac{2 \pi \omega_{0}}{L}} \vec{e}_{\sigma} u_{m}\left(\vec{r}_{\perp} ; z\right) \mathrm{e}^{i k z} a_{L, m \sigma k}+\text { H.C. }
$$

Throughout this review we set $\hbar=1$. $L$ is the length of the quantization volume, and the sum is over the polarization $\sigma$, the transverse mode number $m$, as well as the longitudinal wave vector $k$. The mode functions $u_{m}\left(\vec{r}_{\perp} ; z\right)$, where $\vec{r}_{\perp}=(x, y)$, describes the transverse profile of the beam, form a complete orthogonal set in the plane transverse to the propagation direction

$$
\int d^{2} \vec{r}_{\perp} u_{m}^{*}\left(\vec{r}_{\perp} ; z\right) u_{m^{\prime}}\left(\vec{r}_{\perp} ; z\right)=\delta_{m, m^{\prime}}
$$

In the above we have assumed that the fields belong to a narrow frequency band such that for all modes the frequency under the square root is $\omega_{0}$. Secondly, the field should in general be expanded into a complete set of modes $\vec{u}_{k}(\vec{r})$, but in the paraxial approximation, we have assumed that we can factor out a polarization vector $\vec{e}_{\sigma}$ as well as ignore the $k$ dependence of the transverse mode function $u_{m}\left(\vec{r}_{\perp} ; z\right)$.

Instead of using longitudinal wave vectors we use a slowly varying position space annihilation operator defined by

$$
a_{L, m \sigma}(z)=\sqrt{\frac{c}{L}} \sum_{k} \mathrm{e}^{i\left(k-k_{0}\right) z+i \omega_{0} t} a_{L, m \sigma k}
$$

where $c$ is the speed of light and $k_{0}=\omega_{0} / c$. In the continuum limit $L \rightarrow \infty$ this operator has the commutation relation

$$
\left[a_{L, m \sigma}(z), a_{L, m^{\prime} \sigma^{\prime}}^{\dagger}\left(z^{\prime}\right)\right]=c \delta_{m, m^{\prime}} \delta_{\sigma, \sigma^{\prime}} \delta\left(z-z^{\prime}\right) .
$$

Note that we have chosen here a normalization with $c$ appearing in the commutator. With this normalization (i) the travelling fields $a_{L, m \sigma}(z, t)=a_{L, m \sigma}(z-$ $c t)$ considered below have the commutation relation 
appropriate for operators which are a function of $t$ $\left(\left[a_{L, m \sigma}(z, t), a_{L, m^{\prime} \sigma^{\prime}}\left(z, t^{\prime}\right)\right]=\delta_{m, m^{\prime}} \delta_{\sigma, \sigma^{\prime}} \delta\left(t-t^{\prime}\right)\right)$, and (ii) with this normalization $a_{L, m \sigma}(z)^{\dagger} a_{L, m \sigma}(z)$ describes the flux of photons in mode $m$ with polarization $\sigma$ at position $z$.

In terms of this operator the electric field is given by

$\vec{E}(\vec{r})=\sqrt{\frac{2 \pi \omega}{c}} \sum_{m, \sigma} \vec{e}_{\sigma} u_{m}\left(\vec{r}_{\perp} ; z\right) \mathrm{e}^{i\left(k_{0} z-\omega_{0} t\right)} a_{L, m \sigma}(z)+$ H.C.

Below we mainly deal with a single transverse field mode and a single polarization, so that the sum in the expression above can be omitted. For an introductory textbook to continuous mode quantum optics we refer to (Loudon, 2004).

Atoms We first discuss the theory for atoms with two stable ground states $|0\rangle$ and $|1\rangle$. In subsec. II.D we show how one can in many cases reduce the description for multilevel atoms to two state atoms. The two ground states are conveniently described in terms of angular momentum operators. We shall here use the $x$ as the quantization axis for consistency with Julsgaard et al. (2001, 2004a) and Sherson et al. (2006c). The angular momentum operators describing the $m$ th atom are

$$
\begin{aligned}
j_{x, m} & =\frac{1}{2}\left(|0\rangle_{m}\langle 0|-| 1\rangle_{m}\langle 1|\right), \\
j_{+, m} & =j_{y, m}+i j_{z, m}=|0\rangle_{m}\langle 1|,
\end{aligned}
$$

where $j_{+, m}$ is the operator which raises $j_{x, m}$ by unity.

We shall here be interested in collective variables for an ensemble containing many atoms. In the simplest case such collective operators are given by the total angular momentum operators $J_{l}=\sum_{m} j_{l, m}$ (with $\left.l=x, y, z\right)$, which fulfil the standard angular momentum commutation relation

$$
\left[J_{y}, J_{z}\right]=i J_{x} .
$$

The state with all atoms in state $|0\rangle$, then corresponds to the state $\left|J=N_{A} / 2, M_{x}=N_{A} / 2\right\rangle$ with total angular momentum quantum number $J=N_{A} / 2$ and an eigenvalue of $J_{x}$ equal to $N_{A} / 2$, where $N_{A}$ is the number of atoms. If we consider a large number of atoms and we only weakly perturb the system (only change the state of a few atoms), we can approximate the $J_{x}$ operator by its expectation value $J_{x} \approx\left\langle J_{x}\right\rangle$. For readers who fell uneasy about replacing an operator by its mean value, a more stringent formulation can be made by using the so called Holstein-Primakoff transformation (Holstein and Primakoff, 1940, Kittel, 1987) or in terms of a Wigner group contraction (Arecchi et al., 1972 ). Without loss of generality we can assume the expectation value $\left\langle J_{x}\right\rangle$ to be positive and we can then introduce new canonical position and momentum operators by

$$
X_{A}=\frac{J_{y}}{\sqrt{\left\langle J_{x}\right\rangle}}, \quad P_{A}=\frac{J_{z}}{\sqrt{\left\langle J_{x}\right\rangle}} .
$$

From 15e immediately see that these operators satisfy the standard commutation relation for position and momentum (1). The collective annihilation operator is then

$$
a_{A}=\frac{X_{A}+i P_{A}}{\sqrt{2}}=\frac{\sum_{m} j_{+, m}}{\sqrt{2\left\langle J_{x}\right\rangle}}=\frac{\sum_{m}|0\rangle_{m}\langle 1|}{\sqrt{2\left|\left\langle J_{x}\right\rangle\right|}} .
$$

To get a feeling for this operator, let us consider the action of the creation operator $a_{A}^{\dagger}$. If we apply this operator to the initial state, where all atoms are in state $|0\rangle$, we create a symmetric superposition of one atom being flipped

$$
a_{A}^{\dagger}|0,0,0, \ldots, 0\rangle=\frac{1}{\sqrt{N_{A}}} \sum_{m}\left|0,0, \ldots, 0,1_{m}, 0, \ldots, 0\right\rangle .
$$

Here $\left|0,0, \ldots, 0,1_{m}, 0, \ldots, 0\right\rangle$ is the state, where all atoms except the $m$ th atom are in state $|0\rangle$.

The collective operators above are convenient for describing the entire ensemble. We shall, however, also be dealing with situations were we need to consider collective operators which do not have equal weight on all atoms (Kuzmich and Kennedy, 2004). In the literature such situations are often described by dividing the ensemble into small boxes and constructing collective operators for each box (Fleischhauer and Richter, 1995; Raymer and Mostowski, 1981).

Here we shall use a slightly different formalism (Sørensen and Sørensen, 2008). For a collection of atoms at positions $\vec{r}_{1}, \vec{r}_{2}, \ldots, \vec{r}_{N_{A}}$ we define the classical number density operator

$$
n(\vec{r})=\sum_{m} \delta\left(\vec{r}-\vec{r}_{m}\right)
$$

In condensed matter physics this operator is used to describe scattering from structures (Chaikin and Lubensky, 1995): averaging this density operator over the random positions of the atoms gives the average number density of the atoms $\bar{n}(\vec{r})=\langle n(\vec{r})\rangle$, whereas higher order correlations like $\left\langle n(\vec{r}) n\left(\vec{r}^{\prime}\right)\right\rangle$ describe the correlation responsible for Bragg scattering. Similarly we may introduce continuous atomic spin operators by

$$
j_{k}(\vec{r})=\sum_{m} \delta\left(\vec{r}-\vec{r}_{m}\right) j_{k, m}
$$

where $k=x, y, z,+,-$. A position dependent atomic annihilation operator can then be introduced by

$$
a_{A}(\vec{r})=\frac{1}{\sqrt{2\left\langle j_{x}(\vec{r})\right\rangle}} j_{+}(\vec{r})
$$

where the average spin density $\left\langle j_{x}(\vec{r})\right\rangle$, which we assume to be positive, is the quantum mechanical expectation value averaged over the random position of the atoms. The annihilation operator has the commutation relation

$$
\left[a_{A}(\vec{r}), a_{A}^{\dagger}\left(\vec{r}^{\prime}\right)\right]=\delta\left(\vec{r}-\vec{r}^{\prime}\right) \frac{j_{x}(\vec{r})}{\left\langle j_{x}(\vec{r})\right\rangle} \approx \delta\left(\vec{r}-\vec{r}^{\prime}\right),
$$


where we in the last step have assumed that the fluctuations in the mean spin are much smaller than its average. We shall use this approximation throughout this article. The approximation we are making can thus be seen as a BBGKY approximation, where one truncates an infinite set of equations by replacing an operator by its mean value.

The operators (21) will be convenient for describing the spatial dependence of various operators. To relate them to single mode operators such as Eq. (17), we can introduce a normalized set of mode functions $u_{n}(\vec{r})$ fulfilling the orthogonality and completeness relations in Eq. 66.

We can then construct single mode operators as in Eq. 77. In particular let us consider the normalized mode $u_{\text {sym }}(\vec{r})=\sqrt{\left\langle j_{x}(\vec{r})\right\rangle /\left\langle J_{x}\right\rangle}$. If we use this mode to construct a collective operator, we see from Eq. (21) that this produces the symmetric operator defined in Eq. (17).

The $j_{x}(\vec{r})$ operator will appear in the Hamiltonians below, although sometimes it is more convenient to have expressions which only involve the annihilation operator $a_{A}(\vec{r})$. To find the equations of motion we need to take the commutator of $a_{A}(\vec{r})$ with the Hamiltonian, but from the commutation relation

$$
\left[a_{A}(\vec{r}), j_{x}(\vec{r})\right]=-\frac{1}{\sqrt{2\left\langle j_{x}(\vec{r})\right\rangle}} \sum_{m} j_{+, m}=-a_{A}(\vec{r}),
$$

we see that we get the same result if we make the replacement

$$
j_{x}(\vec{r}) \rightarrow \frac{n}{2}-a_{A}^{\dagger}(\vec{r}) a_{a}(\vec{r})
$$

and use the commutation relation in Eq. 222 (the first term is included to ensure that $j_{x}$ has the right value in the vacuum state of $a_{A}$, where all atoms are in state $|0\rangle)$. This replacement holds even as an exact operator identity in the framework of the Holstein-Primakoff transformation (Holstein and Primakoff, 1940).

\section{B. Interaction of light with model atoms}

We consider atoms with stable ground states denoted by $\left|g_{m}\right\rangle$ and excited states denoted by $\left|e_{m}\right\rangle$. The Hamitonian $H=H_{\mathrm{L}}+H_{\mathrm{A}}+H_{\text {int }}$ describing this system is the sum of the field energy $H_{\mathrm{L}}$, the atomic energy $H_{\mathrm{A}}$, and the interaction Hamiltonian $H_{\text {int }}$. Below we first consider the atomic and interaction parts of the Hamiltonian and derive an effective interaction Hamiltonian involving only the ground states of the atoms. We then include the Hamiltonian $H_{\mathrm{L}}$ responsible for the propagation of the light field, and derive coupled equations of motion for the light and atomic operators.

Interaction with a single atom To describe the atomic part and the interaction let us first consider only a single atom at location $\vec{r}$. In a rotating frame with respect to the laser frequency the atomic Hamiltonian is given by $H_{\mathrm{A}}=$ $\sum_{m} \Delta_{m}\left|e_{m}\right\rangle\left\langle e_{m}\right|$, where $\Delta_{m}$ is the detuning of the $m$ th excited state with respect to the laser frequency.

In the dipole approximation the interaction between light and atoms is described by the Hamiltonian $H_{\text {int }}=$ $\vec{E} \cdot \vec{D}$, where $\vec{D}$ is the electric dipole operator for the atom. In Appendix IX.A we describe the adiabatic elimination of the excited state and derive an effective ground state Hamiltonian

$$
H_{\mathrm{int}}=\sum_{m, m^{\prime}} V_{m^{\prime}, m}(\vec{r})\left|g_{m^{\prime}}\right\rangle\left\langle g_{m}\right|,
$$

where the coupling matrix $V_{m^{\prime}, m}$ is given by

$V_{m^{\prime}, m}(\vec{r})=-\sum_{m^{\prime \prime}} \frac{\left(\vec{E}^{(-)}(\vec{r}) \cdot \vec{D}_{m^{\prime}, m^{\prime \prime}}^{(+)}\right)\left(\vec{D}_{m^{\prime \prime}, m}^{(-)} \cdot \vec{E}^{(+)}(\vec{r})\right)}{\Delta_{m^{\prime \prime}}}$.

Here superscripts $(+)$ and $(-)$ refer to the positive and negative frequency components of the electric field and dipole operators (the positive frequency part, is the part of the operators which removes an excitation, e.g. $\left.\vec{D}_{m^{\prime}, m}^{(+)}=\left\langle g_{m^{\prime}}|\vec{D}| e_{m}\right\rangle\right)$ Loudon 2004.

Interaction with many atoms Let us now consider the situation, where we have many atoms. We are mainly interested in describing the interaction of a weak quantum field with an ensemble driven by a strong classical field. In this subsection we only describe the interaction between the atoms and the forward propagating quantum fields and ignore the spontaneous emission due the coupling to all other e.-m. modes. We shall include the decoherence caused by spontaneous emission in subsec. II.C. To simplify the theory we shall only derive equations of motion for the initially fully polarized ensemble $\left\langle J_{x}(\vec{r}, t)\right\rangle \approx n(\vec{r}) / 2$ (we ignore the difference between the density operator $n(\vec{r})$ and it average value $\bar{n}(\vec{r}))$. The atomic operators defined in Eq. (21) are, however, well behaved annihilation operators even for an ensemble, which is not fully polarized provided that the fluctuations of the mean spin are small. This will for instance be the case if an ensemble containing many atoms is prepared with imperfect optical pumping.

The interaction Hamiltonian can be obtained by summing the single atom Hamiltonian (25) over all atoms. This sum may be replaced by an integral by introducing the continuous atomic annihilation operator defined in Eq. 21)

$$
\begin{aligned}
H=\int d^{3} \vec{r} & {\left[\left(\sqrt{n(\vec{r})} a_{A}(\vec{r}) V_{01}(\vec{r})+\text { H.C. }\right)\right.} \\
& \left.+n(\vec{r}) V_{00}+a_{A}^{\dagger}(\vec{r}) a_{A}(\vec{r})\left(V_{11}-V_{00}\right)\right],
\end{aligned}
$$

where we have used the replacement (24) for the spin operator $j_{x}(\vec{r})$. In Appendix IX.B we use this general 
Hamiltonian to derive Hamiltonians for the three different model systems in Fig. 1 (b)-(d).

For some situations the 3D Hamiltonians derived in Appendix IX.B can be reduced to one dimension. Let us perform this reduction for the beam splitter interaction Hamiltonian in Eq. 95), corresponding to the level configuration as shown in Fig. 2(a), where both classical and quantum field is traveling in the $z$ direction. Lets us now assume that the density is independent of the transverse coordinate $n(\vec{r})=n(z)$, and that the classical laser field is also constant transverse to the propagation direction. Since the mode functions $u_{m}\left(\vec{r}_{\perp} ; z\right)$ form a complete set in the plane, we can expand the atomic operator $a_{A}(\vec{r})$ in the same set

$$
a_{A}(\vec{r})=\sum_{m} u_{m}\left(\vec{r}_{\perp} ; z\right) a_{A, m}(z)
$$

where

$$
a_{A, m}(z)=\int d^{2} \vec{r}_{\perp} u_{m}^{*}\left(\vec{r}_{\perp} ; z\right) a_{A}(\vec{r})
$$

These new operators will then have the appropriate commutation relation $\left[a_{A, m}(z), a_{A, m^{\prime}}^{\dagger}\left(z^{\prime}\right)\right]=\delta\left(z-z^{\prime}\right) \delta_{m, m^{\prime}}$. We insert this expansion into the Hamiltonian (95), and then use the orthogonality relation (9) integrate over the transverse coordinate to obtain the one dimensional Hamiltonian

$$
\begin{aligned}
H_{B S}= & \int d z\left[\frac{-|\Omega(z, t)|^{2}}{4 \Delta} \sum_{m} a_{A, m}^{\dagger}(z) a_{A, m}(z)\right. \\
& -\frac{|g(z)|^{2}}{\Delta} \sum_{m} a_{L, m}^{\dagger}(z) a_{L, m}(z) \\
& \left.-\left(\frac{g^{*}(z) \Omega(z, t)}{2 \Delta} \sum_{m} a_{L, m}^{\dagger}(z) a_{A, m}(z)+\text { H.C. }\right)\right],
\end{aligned}
$$

where the the coupling constant $g(z)$ and slowly varying resonant Rabi frequency $\Omega(z, t)$ are given by

$$
\begin{aligned}
g(z) & =\sqrt{\frac{2 \pi \omega n(z)}{c}} D_{0}, \\
\Omega(z, t) & =2 \vec{D}_{e, 1}^{(-)} \cdot\left\langle\vec{E}^{(+)}\right\rangle \exp \left(-i\left(k_{0} z-\omega_{0} t\right)\right)
\end{aligned}
$$

and the dipole element for the $|0\rangle-|e\rangle$ transition is given by $D_{0}=\vec{D}_{e, 0}^{(-)}$. The Hamiltonian above consists of three terms: the first line is the AC-Stark shift of the atomic ground state, the second is the index of refraction of the gas, and the last line, which is the most important for our discussion here, describes the exchange of excitations between atoms and light.

Note that each of the transverse modes of the light field in (30) talks to a single transverse mode of the atoms, which then couples back to the same transverse light mode. Since the dynamics is actually the same for all the involved transverse modes, the atomic ensembles

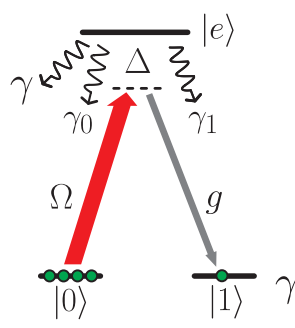

(a)

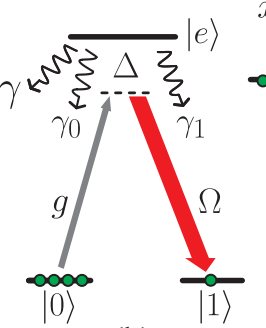

(b)

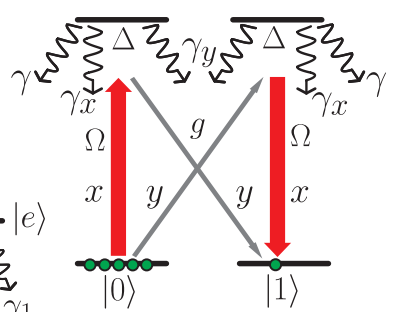

(c)
FIG. $2 \Lambda$ configuration with dominant population of level $|0\rangle$ : (a) Beam splitter interaction with quantum field of single photon Rabi frequency $g$ on the $|0\rangle \rightarrow|e\rangle$ and laser light with Rabi frequency $\Omega$ on the $|e\rangle \rightarrow|1\rangle$ transition. Fields are in two-photon resonance with a detuning $\Delta$ from the excited state $|e\rangle$. Decay due to spontaneous emission goes back to one of the ground states at rates $\gamma_{0(1)}$ or to other levels (not shown) at rate $\gamma$. (b) Paramteric gain interaction. (c) Faraday interaction: Atoms are polarized to $|0\rangle$, laser light is linear polarized along $x$ and drives the up-transitions with a Rabi frequency $\Omega$ and the quantum field in $y$ polarization couples to the cross-transitions with a single photon frequency $g$. Fields are in two photon resonance with a detuning $\Delta$ from the excited states. Decay due to spontaneous emission goes back to one of the ground states at rates $\gamma_{x(y)}$ or to other levels (not shown) at rate $\gamma$.

may in fact be used as a memory for for multiple transverse modes (Camacho et al., 2007, Shuker et al., 2007; Vasilyev et al. 2008, Vudyasetu et al. 2008). A similar description of the reduction from three to one dimension is also presented in André (2005).

We have here used the same central frequency $\omega_{0}$ for both the quantum and classical field, which is true for degenerate $|0\rangle$ and $|1\rangle$ states. If they are not degenerate the energy difference between the two states can be accounted for by changing the frequency $\omega_{0}^{\prime}$ of the classical field. In this case, however, an additional phase factor $\exp \left(i\left(k_{0}^{\prime}-k_{0}\right) z\right)$ associated with the difference of the $k$ vectors appears. We will discuss the effect of this phase factor in Sec. [I.G and Sec. VII

For the parametric gain Hamiltonian (96), cf. Fig. 2(b), the reduction from three to one dimension can be achieved using the same procedure as above. The only difference is that instead of Eq. 29, we should now define the discrete atomic operator by

$$
a_{A, m}(z)=\int d^{2} \vec{r}_{\perp} u_{m}\left(\vec{r}_{\perp} ; z\right) a_{A}(\vec{r}) .
$$

Although the omission of the complex conjugate in this expression compared to Eq. (29) may seem of minor importance, it actually does play a role for several experiments as we will discuss in Sec. IV.C. Omitting the sum 
over multiple modes, the Hamiltonian thus reduces to

$$
\begin{aligned}
H_{G}= & \int d z\left[\frac{|\Omega(z, t)|^{2}}{4 \Delta} a_{A}^{\dagger}(z) a_{A}(z)\right. \\
& \left.-\left(\frac{g^{*}(z) \Omega(z, t)}{2 \Delta} a_{L}^{\dagger}(z) a_{A}^{\dagger}(z)+\text { H.C. }\right)\right] .
\end{aligned}
$$

Finally we derive the one dimensional Hamiltonian for the QND (Faraday) interaction as shown in Fig. 2(c), where the $x$-polarized classical field couple the vertical transitions and a quantum field in $y$ polarization coupe diagonal transitions. From the figure we see that the Faraday interaction is essentially a combination of the beam splitter and the parametric gain interaction. In fact, as discussed in Appendix IX.B the three dimensional Hamiltonian for the Faraday interaction is simply $H_{F}=\left(H_{B S}-H_{G}\right) / \sqrt{2}$. To reduce the problem to one dimension we would like to introduce atomic operators similar to Eqs. 29 and 32 , but now there is an ambiguity as to which of the two forms one should use. We assume that the mode functions $u_{m}\left(\vec{r}_{\perp} ; z\right)$ are real [for Hermite-Gaussian modes (Milonni and Eberly, 1988), this condition can only be satisfied if the Fresnel number is much greater than unity $F=w_{0}^{2} / \lambda L \gg 1$, where $w_{0}$ is the beam waist and $\lambda$ is the wavelength of light (Müller et al., 2005; Sørensen and Sørensen, 2008)], and that $g^{*}(\vec{r}) \Omega(\vec{r}, t)$ has a constant phase, which we take to be zero. With these assumptions the Hamiltonian can be reduced to the simple form

$$
H_{F}=-\int d z \frac{g^{*}(z) \Omega(z, t)}{\sqrt{2} \Delta} p_{L}(z) p_{A}(z),
$$

We have here omitted the index of refraction of the gas for the reasons discussed in Appendix IX.B.

We emphasize that the above $3 \mathrm{D}$ to $1 \mathrm{D}$ reduction provides a very simplified treatment of the propagation of light through an atomic gas. In particular a general treatment should include the spontaneous emission, the density-density correlation of the atoms and the optically induced dipole-dipole interaction of the atoms. For the Faraday interaction a detailed study of the reduction from three to one dimension is presented by Sørensen and Sørensen (2008). In essence this study confirms the treatment presented here provided that the gas is ideal and that the modefunctions $u_{m}\left(\vec{r}_{\perp} ; z\right)$ are the solutions to the propagation equation including the index of refraction. Little work has been done so far on the optically induced dipole interactions.

Equations of motion The addition of the light Hamiltonian $H_{L}$ to the atomic part of the Hamiltonian $H_{A}$ and the interaction $H_{\text {int }}$ discussed above allows to describe the propagation of light through the ensemble. As shown in Appendix IX.C the equation of motion is derived by introducing a rescaled time $\tau=t-z / c$ and becomes a differential equation in space $(z)$ instead of time $t$.
For the beam splitter interaction, following this recipe, we find the equations of motion by calculating the commutator with $H_{B S}$

$$
\begin{aligned}
\frac{\partial}{\partial z} a_{L}(z, t) & =i \frac{|g(z)|^{2}}{\Delta} a_{L}(z, t)+i \frac{g^{*}(z) \Omega(t)}{2 \Delta} a_{A}(z, t), \\
\frac{\partial}{\partial t} a_{A}(z, t) & =i \frac{|\Omega(t)|^{2}}{4 \Delta} a_{A}(z, t)+i \frac{g(z) \Omega^{*}(t)}{2 \Delta} a_{L}(z, t) .
\end{aligned}
$$

The above equations can be solved analytically (Gorshkov et al. , 2007c; Kozhekin et al., 2000, Kupriyanov et al. | 2005: Mishina et al., 2007, Nunn et al., 2007). We will defer a discussion of the solutions till Sec. II.C where the spontaneous emission is included.

Similarly we can find the equations of motion for the parametric gain and we get

$$
\begin{aligned}
\frac{\partial}{\partial z} a_{L}(z, t) & =i \frac{g^{*}(z) \Omega(z, t)}{2 \Delta} a_{A}^{\dagger}(z, t) \\
\frac{\partial}{\partial t} a_{A}(z, t)=- & i \frac{|\Omega(z, t)|^{2}}{4 \Delta} a_{A}(z, t) \\
& +i \frac{g^{*}(z) \Omega(z, t)}{2 \Delta} a_{L}^{\dagger}(z, t)
\end{aligned}
$$

Again these equations have an analytical solution (Carman et al., 1970, Raymer and Mostowski, 1981).

For the Faraday interaction the equations of motion are much simpler when expressed in terms of $x$ and $p$ and read

$$
\begin{aligned}
\frac{\partial}{\partial z} x_{L}(z, t) & =-\frac{g^{*}(z) \Omega(t)}{\sqrt{2} \Delta} p_{A}(z, t), \\
\frac{\partial}{\partial z} p_{L}(z, t) & =0, \\
\frac{\partial}{\partial t} x_{A}(z, t) & =-\frac{g^{*}(z) \Omega(t)}{\sqrt{2} \Delta} p_{L}(z, t), \\
\frac{\partial}{\partial t} p_{A}(z, t) & =0 .
\end{aligned}
$$

Because the two momentum operators are conserved quantities these equations describe a QND interaction, where one can, e.g., make a measurement of the position operator $x_{L}$ after the interaction and thereby obtain a QND measurement of the atomic momentum operator $p_{A}$, as will be further explained in Sec. IV.A.

The presence of the conserved quantities $p_{A}$ and $p_{L}$ makes it straightforward to solve the equations of motion. The only $z$ dependence in the above expressions comes from the $z$ dependence of the density $n(z)$. We can then define the symmetric operators by

$$
\begin{aligned}
X_{A} & =\frac{\int d z \sqrt{n(z)} x_{A}(z)}{\sqrt{\int d z n(z)}}, \\
P_{A} & =\frac{\int d z \sqrt{n(z)} p_{A}(z)}{\sqrt{\int d z n(z)}} .
\end{aligned}
$$


If the transverse mode function includes all the atoms in the ensemble, these operators are equivalent to the symmetric operators defined in Eq. (16). Similar integrated operators for the light field are

$$
\begin{aligned}
& X_{L}=\frac{\int d t \Omega(t) x_{L}(t)}{\sqrt{\int d t \Omega^{2}(t)}}, \\
& P_{A}=\frac{\int d t \Omega(t) p_{L}(t)}{\sqrt{\int d t \Omega^{2}(t)}},
\end{aligned}
$$

where we assume that $\Omega$ is real. Expressed in terms of these variable the equations of motions have the simple solutions

$$
\begin{aligned}
X_{L, \text { out }} & =X_{L, \text { in }}+\kappa P_{A, \text { in }}, \\
P_{L, \text { out }} & =P_{L, \text { in }}, \\
X_{A, \text { out }} & =X_{A, \text { in }}+\kappa P_{L, \text { in }}, \\
P_{A, \text { out }} & =P_{A, \text { in }} .
\end{aligned}
$$

The subscript "in" and "out" means input and output variables, e.g., $X_{L \text {,in }}=X_{L}(z=0)$ and $X_{L \text {,out }}=X_{L}(z=$ $L)$ for light and $X_{A \text {,in }}=X_{A}(t=0)$ and $X_{A \text {,out }}=X_{A}(t=$ $T$ ) for atoms. The coupling constant $\kappa$ is given by

$$
\begin{aligned}
\kappa^{2} & =\int d t \frac{|\Omega(t)|^{2}}{2 \Delta^{2}} \int d z|g(z)|^{2} \\
& =\frac{\pi \omega D_{-}^{2}}{c} \int d t \frac{|\Omega(t)|^{2}}{\Delta^{2}} \int d z n(z) .
\end{aligned}
$$

In the last line we have inserted the expression for the coupling constant $g(z)(31)$ and have denoted the dipole matrix element by $D_{-}$to indicate that it is the coupling constant for $\sigma$ - polarized light. As we shall see the coupling constant $\kappa$ (and the generalization of it) will play an important role for characterizing the strength of the interaction regardless of the level scheme being used. Note, that $\kappa$ only depends on the total integrated density. This property can be shown to apply also to the $\Lambda$ schemes by a simple rescaling of the $z$ coordinate (Gorshkov et al. 2007c). We shall therefore for simplicity only consider a constant density below.

\section{Theory Including Spontaneous emission}

In subsec. II.B we derived the equations of motion describing the interaction between light and ensembles of atoms neglecting the spontaneous emission from the atoms. In this section we will give the theory including spontaneous emission and discuss the solutions to the equations.

Instead of repeating the calculations in subsec. II.B now with a non-zero decay, we note that if the spontaneous emission from each atom is independent, it can be accounted for by making the substitution

$$
\Delta_{m} \rightarrow \Delta_{m} \pm i \frac{\gamma_{m}}{2}
$$

in all the calculations performed so far. The choice of the sign is discussed in the Appendix IX.D and the results are stated below. It is also important to note that the quantity $\left\langle j_{x}(\vec{r})\right\rangle$ used to define the operator $a_{A}(\vec{r}, t)$ in Eq. (21), is not necessarily a constant. To ensure that the operator $a_{A}(\vec{r}, t)$ has the right normalization we should always normalize by the time dependent expectation value $\left\langle j_{x}(\vec{r}, t)\right\rangle$. The time derivative of $\left\langle j_{x}(\vec{r}, t)\right\rangle$ will introduce extra terms as discussed in Appendix IX.D.

Beam splitter interaction As discussed in Appendix IX.D for the beam splitter interaction we should use the minus sign in the substitution (42), and the time derivative of $\left\langle j_{x}(\vec{r})\right\rangle$ can be neglected because the strong classical field talks to an almost empty level. For a constant atomic density the equations of motion then become

$$
\begin{aligned}
\frac{\partial}{\partial z} a_{L}(z, t) & =\frac{i|g|^{2}}{\Delta-i \frac{\gamma}{2}} a_{L}(z, t)+\frac{i g^{*} \Omega(t)}{2 \Delta-i \gamma} a_{A}(z, t), \\
\frac{\partial}{\partial t} a_{A}(z, t) & =\frac{i|\Omega(t)|^{2}}{4\left(\Delta-i \frac{\gamma}{2}\right)} a_{A}(z, t)+\frac{i g \Omega^{*}(t)}{2 \Delta-i \gamma} a_{L}(z, t),
\end{aligned}
$$

where we have omitted the noise operators given in Eq. (105). The admixed noise is vacuum in both equations of motion, and since the equations only couple annihilation operators to annihilation operators we can ignore the noise for any normally ordered products Gorshkov et al. 2007b).

Solving these equation for a resonant field $\Delta=0$ with no classical field $\Omega=0$ we find that the intensity is reduced by a factor of $\exp (-d)$, with the optical depth $d$ given by

$$
d=L \frac{4|g|^{2}}{\gamma}=\frac{3 \lambda^{2} \gamma_{0}}{2 \pi \gamma} n L=n \sigma_{0} L
$$

where we have used the expression for the coupling constant $g$ (31) and have introduced the spontaneous decay rate $\gamma_{0}=4 \omega^{3}\left|D_{0}\right|^{2} / 3 c^{3}$ from the excited state $|e\rangle$ into the ground state $|0\rangle$ (Milonni and Eberly, 1988) and the absorption cross section for an atom $\sigma_{0}=3 \lambda^{2} \gamma_{0} / 2 \pi \gamma$ (Jackson, 1975).

It is sufficient in our case to solve the operator equations of motion (43) as "classical equations" with the operators replaced by complex functions (Gorshkov et al. 2007b; Raymer and Mostowski, 1981). The reason is that the equations are linear in the operators. The solutions to the operator equations will therefore be of the form

$$
\hat{a}_{L, \text { out }}(t)=\int_{0}^{L} d z m\left(\Omega\left(t^{\prime}\right) ; t, L-z\right) \hat{a}_{A, \text { in }}(z)+\ldots .
$$

where we have included the hats on the operators for clarity, and where we include the argument $\Omega\left(t^{\prime}\right)$ to indicate that the solution depends on the driving field at all times. The remaining terms denoted by dots in Eq. (45) are similar linear combinations of the input light and 
noise operators. If we for instance solve the equations of motion with a complex function $a_{A \text {,in }}(z)$ as an initial condition, we will due to the linearity of the equations get the same solution only without the hats

$$
a_{L, \text { out }}(t)=\int_{0}^{L} d z m(\Omega(t), t, L-z) a_{A, \text { in }}(z) .
$$

We can thus obtain most of the solution 45 back by inserting hats in the solution. Because we have ignored some input operators, however, the resulting operators will not necessarily have the right commutation relation. If there is no incident light all of these other modes will be in vacuum, and one can obtain the right commutation relation by adding a suitable amount of vacuum noise (Gorshkov et al., 2007b). Another way to see why the complex number equations are sufficient to obtain full information about the dynamics is to note that if all input modes are in classical coherent states we can take expectation values of the equations of motion (43) and obtain the same equations of motion for the mean values. These classical equations of motion are thus identical to the quantum equations of motion (Raymer and Mostowski, 1981). The equations of motion correspond to a general beam splitter relation, so that with coherent states as input states the output quantum states will also be a set of coherent states with amplitudes given by the mean values. Since any initial state can be expanded on the set of coherent states, the knowledge about the evolution of the coherent states obtained by solving the equations of motion for the mean values gives us complete information about the evolution for any quantum state.

The equations of motion can in fact be solved analytically (Gorshkov et al., 2007c). If we consider the situation where the only non vanishing initial value is $a_{A}$ we find the solution for the output light in Eq. (46) with

$$
\begin{aligned}
m\left(\Omega\left(t^{\prime}\right) ; t, z\right)= & \sqrt{\frac{\gamma d}{L}} \frac{(-i) \Omega(z, t)}{4\left(\Delta-i \frac{\gamma}{2}\right)} \mathrm{e}^{i\left[\frac{2 \Delta+i \gamma}{2 \gamma} \frac{h(0, t)}{d}+\frac{d \gamma z}{4 L(\Delta-i \gamma / 2)}\right]} \\
& \times I_{0}\left(-i \mathrm{e}^{\mathrm{i} \phi} \sqrt{h(0, t) \frac{z}{L}}\right)
\end{aligned}
$$

where $I_{0}(x)$ denotes the 0th order Bessel function of the first kind. Here we have assumed that the coupling constant is real, which can always be done by absorbing any phase into the definition of $\Omega$, and we have replaced the coupling constant by the optical depth through the relation (44). The function $h\left(t, t^{\prime}\right)$ is defined by

$$
h\left(t, t^{\prime}\right)=\int_{t}^{t^{\prime}} d t^{\prime \prime} \frac{d \gamma\left|\Omega\left(t^{\prime \prime}\right)\right|^{2}}{4 \Delta^{2}+\gamma^{2}},
$$

and as we shall see below, this is also the function characterizing the strength of the Faraday interaction. Finally the phase $\phi$ is defined by

$$
\mathrm{e}^{i \phi}=\frac{\sqrt{4 \Delta^{2}+\gamma^{2}}}{2 \Delta-i \gamma} .
$$

Similarly we find for the output atomic variables

$$
a_{A, \text { out }}(z)=\int_{0}^{T} d t m\left(\Omega^{*}\left(T-t^{\prime}\right) ; T-t, z\right) a_{L, \text { in }}(t) .
$$

The fact that the kernel $m\left(\Omega^{*}\left(T-t^{\prime}\right) ; T-t, z\right)$ is similar to the kernel in Eq. (46) is a direct consequence of time reversal symmetry (Gorshkov et al. $2007 \mathrm{c})$.

While the above solutions are exact for the equations of motion, they are sufficiently complicated and it is hard to gain any physical intuition from them. More insight can be gained by Laplace transforming the equations with respect to space, e.g.,

$$
a_{A}(u, t)=\frac{1}{\sqrt{L}} \int_{0}^{\infty} d z \mathrm{e}^{-u z / L} a_{A}(z, t),
$$

where we have chosen the normalization so that $u$ is a dimensionless number of order unity. We can then derive the equations of motion for the Laplace transformed variables. Since the Laplace transform makes the derivative with respect to $z$ simple, we can then eliminate the light field and obtain a single equation for the atomic operator

$$
\begin{aligned}
\frac{\partial}{\partial t} a_{A}(u, t)= & \frac{i|\Omega(t)|^{2}}{4\left(\Delta-i \frac{\gamma}{2}\left(1+\frac{d}{2 u}\right)\right)} a_{A}(u, t) \\
& +\frac{i g \sqrt{L} \Omega^{*}(t)}{2 u\left(\Delta-i \frac{\gamma}{2}\left(1+\frac{d}{2 u}\right)\right)} a_{L, \text { in }}(t) .
\end{aligned}
$$

This equation now has a simple interpretation. The real part of the first term, proportional to the detuning $\Delta$ will give rise to an unimportant phase, while the imaginary part describes decay of the atomic excitation at an effective rate (proportional to) $\gamma(1+d / 2 u)$. Here the decay rate $\gamma$ is due to spontaneous emission whereas the second term $\gamma d / 2 u$ is due to the coherent interaction with the forward light mode. For a sufficiently high optical depth $d$ the coherent interaction will dominate the spontaneous emission and we can obtain an efficient interface between atoms and light.

Parametric gain interaction In case of the parametric gain interaction we need to use the plus sign in one of the substitutions as well as include some terms associated with the time derivative of $\left\langle j_{x}(\vec{r}, t)\right\rangle$ as discussed in Appendix IX.D. We then find

$$
\begin{aligned}
\frac{\partial}{\partial z} a_{L}(z, t)= & i \frac{g^{*}(z) \Omega(z, t)}{2 \Delta-i \gamma} a_{A}^{\dagger}(z, t), \\
\frac{\partial}{\partial t} a_{A}(z, t)=- & i \frac{|\Omega(z, t)|^{2} \Delta}{4 \Delta^{2}+\gamma^{2}} a_{A}(z, t) \\
& +i \frac{g^{*}(z) \Omega(z, t)}{2 \Delta-i \gamma} a_{L}^{\dagger}(z, t),
\end{aligned}
$$

where the $z$ dependence of the Rabi-frequency is given by the $\exp \left[i \int d z^{\prime}\left|g\left(z^{\prime}\right)\right|^{2} /(\Delta-i \gamma / 2)\right]$ dependence associated with the change in the propagation of the classical field 
caused by the index of refraction and scattering of the gas. To arrive at these equations, we have assumed that the decay from the excited state goes into some states $\left|a_{m}\right\rangle$ different from $|0\rangle$ and $|1\rangle$, cf. Fig. 2(b). This model for the decay is not always best for real systems used for parametric-type interaction, for example, in DLCZ-type applications (Duan et al., 2001), since a much stronger decay to auxiliary states would decrease the effective optical depth, and a big optical depth is crucial for the interface. The model used here is, however, the simplest possible model, and we therefore restrict ourself to this situation. The reason why there is no decoherence terms in these equations is that spontaneous emission drives atoms from state $|0\rangle$ into some other state $|a\rangle$, where they are lost from the system. Collective states like the one in Eq. 18 are, however, immune to removing one of the atoms in state $|0\rangle$ and this source of decoherence has therefore no effect within the approximations we are using here.

These equations of motion can also be solved analytically (Carman et al., 1970; Raymer and Mostowski, 1981). The solution is similar to the solution for the beam splitter interaction and we shall not go further into it here. Again it can be shown that it is possible to obtain a large coherent coupling with a large optical depth, by Laplace transforming the equations. By doing so one finds a strong gain term for the Laplace component with argument $u$, which is $\sim d / u$ times the single atom scattering rate. For a large optical depth $d$ we are thus dominated by the coherent interaction, regardless of the assumption about the final state after a decay, which we made above. Unlike the beamsplitter interaction, where one can work at any detuning, this large coherent interaction only works in the far off-resonant regime, because the classical beam would be completely depleted if one works on resonance in a gas with large optical depth.

Faraday interaction The Faraday interaction is a combination of the beam splitter interaction and the parametric gain, and the easiest way to obtain the equations of motion, is therefore to just combine the results obtained in the previous two subsections. As discussed in Appendix IX.D the resulting equations of motion are then (assuming $\Omega$ and $g$ to be real)

$$
\begin{aligned}
& \frac{\partial}{\partial z} x_{L}(z, t)=-\frac{2 \sqrt{2} \Delta g(z) \Omega(t)}{4 \Delta^{2}+\gamma^{2}} p_{A}(z)-\frac{\gamma g^{2}}{4 \Delta^{2}+\gamma^{2}} x_{L}(z) \\
& \frac{\partial}{\partial z} p_{L}(z, t)=\frac{\sqrt{2} \gamma g(z) \Omega(t)}{4 \Delta^{2}+\gamma^{2}} p_{A}(z)-\frac{\gamma g^{2}}{4 \Delta^{2}+\gamma^{2}} p_{L}(z) \\
& \frac{\partial}{\partial t} x_{A}(z, t)=-\frac{2 \sqrt{2} \Delta g(z) \Omega(t)}{4 \Delta^{2}+\gamma^{2}} p_{L}(z)-\frac{\gamma \Omega^{2}}{2\left(4 \Delta^{2}+\gamma^{2}\right)} x_{A}(z), \\
& \frac{\partial}{\partial t} p_{A}(z, t)=\frac{\sqrt{2} \gamma g(z) \Omega(t)}{4 \Delta^{2}+\gamma^{2}} p_{L}(z)-\frac{\gamma \Omega^{2}}{2\left(4 \Delta^{2}+\gamma^{2}\right)} p_{A}(z)
\end{aligned}
$$

where we have omitted the noise operators, which are given in Eq. (108). Again these equation are derived under the assumption that the decay goes to some auxiliary state $\left|a_{m}\right\rangle$. For a treatment with decay back to the interface levels, see for instance Hammerer (2006); Madsen and Mølmer (2004).

Let us now consider the solution of Eq. (54) in the limit of a small damping. The Faraday interaction is only used with far off resonant light $\Delta \gg \sqrt{d} \gamma$ since the classical field would be completely absorbed if we were working close to resonance. We will therefore ignore the first term in the evolution of the momentum operators $p_{L}$ and $p_{A}$, which is much smaller than the similar term in the evolution of $x_{L}$ and $x_{A}$. Furthermore, a time dependent driving $\Omega(t)$ can be accounted for by a simple rescaling (see Appendix IX.E), so we only consider a constant driving field $\Omega$. Because the quantities $p_{L}$ and $p_{A}$ appearing in the coupling to the operators $x_{A}$ and $x_{L}$ are conserved quantities apart from the small decays, the dynamics effectively only involve the integrated operators

$$
\begin{aligned}
X_{L} & =\frac{1}{\sqrt{T}} \int_{0}^{T} d t x_{L}(t), \\
P_{L} & =\frac{1}{\sqrt{T}} \int_{0}^{T} d t p_{L}(t), \\
X_{A} & =\frac{1}{\sqrt{L}} \int_{0}^{L} d z x_{A}(z), \\
P_{A} & =\frac{1}{\sqrt{L}} \int_{0}^{L} d z p_{A}(z),
\end{aligned}
$$

where the normalization is chosen as for single mode operators $[X, P]=i$. In the limit of small scattering the resulting dynamics are then given by

$$
\begin{aligned}
X_{L, \text { out }} & \approx \mathrm{e}^{-\eta_{L} / 2} X_{L, \text { in }}+\kappa P_{A, \text { in }}, \\
P_{L, \text { out }} & \approx \mathrm{e}^{-\eta_{L} / 2} P_{L, \text { in }}, \\
X_{A, \text { out }} & \approx \mathrm{e}^{-\eta_{A} / 2} X_{A, \text { in }}+\kappa P_{L, \text { in }}, \\
P_{A, \text { out }} & \approx \mathrm{e}^{-\eta_{A} / 2} P_{A, \text { in }} .
\end{aligned}
$$

Apart from the decay, this solution is the same as the results derived in Eq. (40) with a minor modification of the coupling constant (41) which is now given by

$$
\kappa^{2}=h(0, t) \frac{4 \Delta^{2}}{4 \Delta^{2}+\gamma^{2}},
$$

where the function $h(0, t)$ is the same as the function introduced for the beam splitter interaction in Eq. (48). The coupling constant here is the same as the one derived in the limit of large detuning $\Delta$ in Eq. (41).

In the solutions above, the light and atomic operators are damped by factors of $\exp \left(-\eta_{L} / 2\right)$ and $\exp \left(-\eta_{A} / 2\right)$ respectively. The damping factor for the light is given by

$$
\eta_{L}=\frac{\gamma|g|^{2} L}{4\left(\Delta^{2}+\frac{\gamma^{2}}{4}\right)}=\frac{d}{2} \frac{\gamma^{2}}{4 \Delta^{2}+\gamma^{2}}
$$


Note that the optical depth used here is for linear polarization. The definition of $d$ therefore differs by a factor of 2 from (44) because we have taken $g$ to be the coupling constant for circular polarized light and not for linear polarization. From the expression above it is clear that we can ignore the decay of the light field if we use a sufficiently large detuning $\Delta \gg \sqrt{d} \gamma$. A large detuning also reduces the coupling constant but this can be compensated by using stronger laser fields.

The atomic decoherence can be related to the coupling constant through

$$
d \eta_{A}=\frac{\gamma d \int d t|\Omega|^{2}}{4 \Delta^{2}+\gamma^{2}}=\kappa^{2}
$$

This relation between $d, \eta_{A}$, and $\kappa$ is valid regardless of which assumption one makes about the final state after a decay. But different decay channels will have different effects in the equations of motion, and Eq. (54) is only directly applicable for the particular model considered here. Nevertheless, with a fixed interaction strength $\kappa^{2}$ we can always obtain negligible decoherence with a sufficiently large optical depth $d$.

Scaling with atom and photon number Further insight into the connection between coupling, atomic decoherence and light decay can be obtained by expressing it through the number of atoms $N_{A}$ and photons $N_{P}$ of the classical field. Let us assume that the classical beam has a square profile with a cross section of area $A$. Using the definitions of the electric field $(12)$ and Rabi frequency 31 we find

$$
\int d t|\Omega|^{2}=\gamma_{c} \frac{3 \lambda^{2}}{2 \pi A} N_{P}
$$

where $\gamma_{c}\left(\gamma_{q}\right)$ is the decay rate between the two states coupled by the classical (quantum) field with an emitted photon of the same polarization as the classical (quantum) field. The function $h(0, T)$ characterizing the strength of the interaction in the far off-resonant limit may be then expressed as

$$
\begin{aligned}
h(0, T) & =\left(\frac{3 \lambda^{2}}{2 \pi A}\right)^{2} \frac{\gamma_{c} \gamma_{q}}{4 \Delta^{2}+\gamma^{2}} N_{A} N_{P} \\
& =\frac{\sigma_{c} \sigma_{q}}{A^{2}} \frac{\gamma^{2}}{4 \Delta^{2}+\gamma^{2}} N_{A} N_{P},
\end{aligned}
$$

where in the last line we have introduced the resonant cross sections for the scattering of the quantum and classical fields $\left(\sigma_{m}=3 \lambda^{2} \gamma_{m} / 2 \pi \gamma\right)$. The atomic and light decoherence may then be related by

$$
N_{A} \eta_{A}=N_{P} \eta_{L}
$$

which simply reflects the fact that the number of scattered photons is the same as the number of atoms which have scattered a photon. If the number of input classical photons is much larger than the number of atoms
$N_{P} \gg N_{A}$ the light decay is much smaller than the atomic decoherence and can be neglected.

The function $h(0, t)=\kappa^{2}$ characterizes the solution of all three model systems considered here. Further insight into the reason for this can be gained by rewriting the equations in terms of rescaled dimensionless variables (Appendix IX.E).

\section{Realistic Multilevel Atoms}

In the previous section we derived main equations describing three types of light-matter interactions, the beam splitter, the paramteric gain and the Faraday-QND type, Eqs. 30, 33 and (34) respectively, for simple few-level model atoms. In this section we will give examples for how these interactions are commonly realized with real atoms.

Faraday interaction Applying the Faraday interaction (34) to a full hyperfine level with many nearly degenerate Zeeman states would at first sight seem to violate the simple two level approximation that we have used in the theoretical derivation. For alkali atoms, however, the full theory actually reduces to what we have derived in the previous sections if the detuning is much larger than the hyperfine splitting in the excited state. Consider the $S_{1 / 2} \rightarrow P_{3 / 2}$ transition in alkali atoms as indicated in Fig. 3. If atoms are optically pumped into one of their hyperfine ground state levels $F$, an off resonant probe will couple in general to three dipole allowed transitions $F \rightarrow F^{\prime}=F-1, F, F+1$. Each of these transitions will contribute to the effective Hamiltonian in Eq. 25) describing the interaction of a single atom with off-resonant light. It will be convenient to rewrite this Hamiltonian as

$$
H_{\mathrm{int}}=\vec{E}^{(-)}(\vec{r}) \overleftrightarrow{\alpha} \vec{E}^{(+)}(\vec{r})
$$

where we introduced the atomic polarizability tensor operator (Deutsch and Jessen, 1998, Happer, 1972, Happer and Mathur, 1967)

$$
\overleftrightarrow{\alpha}=\sum_{m, m^{\prime}} \sum_{F^{\prime}} \sum_{m^{\prime \prime}=-F^{\prime}}^{F^{\prime}} \frac{\vec{D}_{m^{\prime}, m^{\prime \prime}}^{(+)} \wedge \vec{D}_{m^{\prime \prime}, m}^{(-)}}{\Delta_{F^{\prime}}}\left|g_{m^{\prime}}\right\rangle\left\langle g_{m}\right|
$$

and $\vec{D}_{m^{\prime}, m^{\prime \prime}}^{(+)} \wedge \vec{D}_{m^{\prime \prime}, m}^{(-)}$denotes the dyadic vector product. The polarizability operator is a rank-2 spherical tensor (Edmonds, 1964, Zare, 1988) and can therefore be decomposed into irreducible tensor components,

$$
\overleftrightarrow{\alpha}=\frac{4 D_{0}^{2}}{\Delta}\left(a_{0}(\Delta) \overleftrightarrow{T}^{(0)}+a_{1}(\Delta) \overleftrightarrow{T}^{(1)}+a_{2}(\Delta) \overleftrightarrow{T}^{(2)}\right)
$$

where the tensor operators $\overleftrightarrow{T}^{(k)}$ transform under rotations as a scalar, vector and matrix for $k=1,2,3$ respectively. In this expression $\Delta=\Delta_{F^{\prime}=F+1}$ is the laser 
detuning from the uppermost level and $2 D_{0}=\left\langle J^{\prime}|| \vec{D} \| J\right\rangle$ is the reduced dipole matrix element for the $S_{1 / 2} \rightarrow P_{3 / 2}$ transition. It relates to the spontaneous decay rate introduced in Sec. II.C as $\gamma_{0}=4 \omega^{3}\left|D_{0}\right|^{2} / 3 c^{3}$.

The real coefficients $a_{k}(\Delta)$ follow from elementary calculations (Geremia et al., 2006, Hammerer, 2006, Julsgaard, 2003) and are given in appendix IX.F The essential feature of these coefficients, which is proven in the appendix IX.F is that for a laser detuning, which is large compared to the hyperfine splitting of excited states, $|\Delta| \gg\left|\Delta_{F+1}-\Delta_{F^{\prime}}\right|$, the rank-2 tensor component vanishes, $a_{2}(\Delta) \rightarrow 0$. For the case of ${ }^{133} \mathrm{Cs}$ the coefficients $a_{k}(\Delta)$ are shown in Fig. 3 .

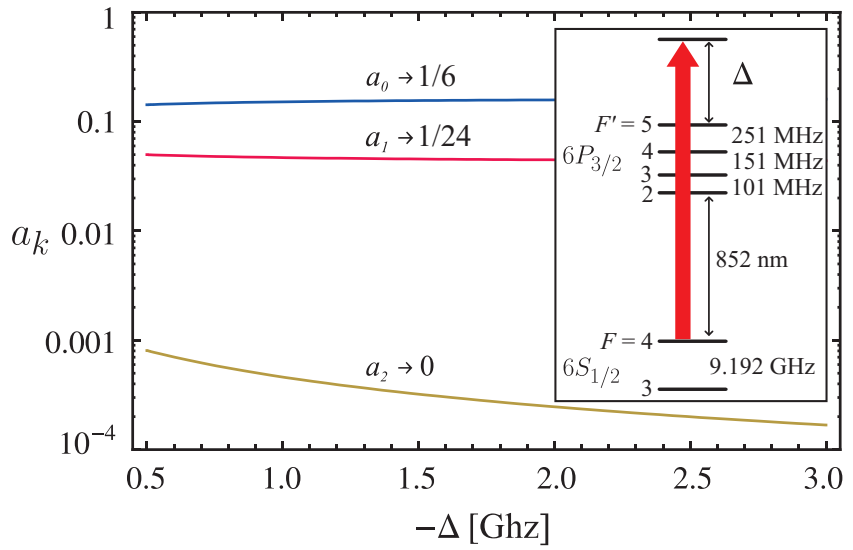

FIG. 3 Coefficients $a_{0}, a_{1}, a_{2}$ for, respectively, scalar, vector and tensor polarizability versus (blue) detuning $\Delta$ of probe light driving the $6 S_{1 / 2} \rightarrow 6 P_{3 / 2}$ transition in ${ }^{133} \mathrm{Cs}$. The inset shows the relevant levels and energy scales.

In the asymptotic limit the interaction Hamiltonian is thus given by

$$
\begin{aligned}
H=\frac{D_{0}^{2}}{\Delta}\left[a_{0} \vec{E}^{(-)}(\vec{r})\right. & \cdot \vec{E}^{(+)}(\vec{r}) \\
& \left.+\frac{i}{2} a_{1} \vec{E}^{(-)}(\vec{r}) \cdot\left(\vec{j} \times \vec{E}^{(+)}(\vec{r})\right)\right] .
\end{aligned}
$$

For an atomic ensemble, the first term will give rise to an index of refraction, while the second term accounts for the Faraday effect. For light propagating along $z$ and the classical light polarized along $x$ the Faraday Hamiltonian (34) can be easily derived.

When the detuning is larger than the hyperfine splitting, the interaction is thus essentially the same as for a spin $1 / 2$ ground state, where $a_{2}$ vanishes exactly for all detunings. This can be understood by looking at how the energy levels of an alkali atom appear. The full Hamiltonian can be written $H=H_{0}+H_{F S}+H_{H F S}+H_{\text {int }}$, where the four Hamiltonians represents the Coulomb, fine structure, hyperfine structure, and interaction with light. Normally one just considers the first three terms as atomic Hamiltonian and does perturbation theory in the interaction Hamiltonian. When the detuning is larger than the hyperfine structure it is, however, more appropriate to do the adiabatic elimination before treating the hyperfine interaction. Without the hyperfine interaction the optical fields only talk to the electron spin, where there cannot be a rank two tensor, since the spin cannot be changed by two, and $a_{2}$ therefore only appears as a perturbation in $H_{H F S} / \Delta$.

$\Lambda$-Systems The beam splitter and the parametric gain interaction, which require a $\Lambda$ configuration in atoms, are commonly implemented taking the two $S_{1 / 2}(F=I \pm 1 / 2)$ hyperfine levels as stable ground states $|0\rangle$ and $|1\rangle$ and one of the $P$ states as excited state $|e\rangle$, see (Chaneliere et al. , 2005, 2007, Chen et al., 2008, 2007; Choi et al. 2008; Chou et al., 2004, 2007, 2005; Matsukevich and Kuzmich, 2004). This approach gives excellent results even if atoms are not optically pumped initially to one Zeeman substate. It has also been suggested by de Echaniz et al. (2008); Kupriyanov et al. (2005); Mishina et al. (2007) to make use of the second rank tensor polarizability to engineer effective $\Lambda$-schemes for the beam splitter and the paramteric gain interactions using two degenerate Zeeman states and polarized light.

\section{E. Ensemble in Magnetic Field}

So far, our theory for light matter interaction assumed degenerate ground state levels. For the parametric gain and beam splitter interaction, having non-degenerate ground states does not make a difference, as the level splitting can be compensated for by choosing appropriate frequencies for light. In this section we will mainly deal with the Faraday interaction for atoms in an external magnetic field causing Zeeman splitting along the axis of polarization (Fig. 4). This can be advantageous in several respects. On the one hand, combined with the homodyne detection typically used in connection with the Faraday interaction, this results in low-noise AC signals, as will be detailed in sec. II.F On the other hand, it can also simplify and enhance protocols aiming for an efficient creation of entanglement of two ensembles (Sec. IV) or between an ensemble and light (Sec. VI).

The free Hamiltonian for an ensemble of atoms in a uniform magnetic field (Eq. (24)) oriented along the $x$ axis is

$$
H_{0}=\frac{\omega_{L}}{2} \int \mathrm{d} z\left(x_{A}^{2}(z)+p_{A}^{2}(z)\right),
$$

where $\omega_{L}$ is the Larmor frequency. This Hamiltonian generates Larmor precession of the transverse spin density components, $x_{A}(z)$ and $p_{A}(z)$, about the $x$-axis. The full Hamiltonian describing Faraday interaction in a magnetic field is $H=H_{0}+H_{F}$, where $H_{F}$ is given in Eq. (34). In an interaction picture with respect to $H_{0}$ this 


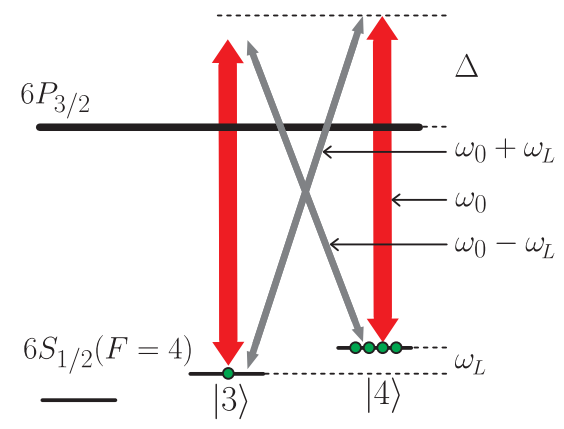

FIG. 4 A magentic field along the axis of polarization causes Zeeman splitting $\omega_{L}$ of ground state levels. Photons will be scattered from the classical light, driving the up transitions, to the quantum field, coupling to the cross transition, at sideband frequencies $\omega_{0} \pm \omega_{L}$ from the carrier frequency $\omega_{0}$.

Hamiltonian is,

$$
\begin{aligned}
H_{F}^{I}=-\int & d z \frac{g^{*}(z) \Omega(z, t)}{\sqrt{2} \Delta} p_{L}(z) \\
& \times\left(\cos \left(\omega_{L} t\right) p_{A}(z)+\sin \left(\omega_{L} t\right) x_{A}(z)\right) .
\end{aligned}
$$

Operators $x_{A}$ and $p_{A}$ refer now to spin components in a frame rotating at $\omega_{L}$ about the $x$ axis. For the sake of simplicity we use in the following the same symbols for canonical operators in both frames, as it will be clear from the context, to which one we are referring. In the rotating frame the canonical operators for transverse spin components are related to the spin components in the lab frame via

$$
\begin{aligned}
& j_{y}(z) / \sqrt{n(z)}=\cos \left(\omega_{L} t\right) x_{A}-\sin \left(\omega_{L} t\right) p_{A}, \\
& j_{z}(z) / \sqrt{n(z)}=\cos \left(\omega_{L} t\right) p_{A}+\sin \left(\omega_{L} t\right) x_{A} .
\end{aligned}
$$

with the number density of atoms $n(z)$.

The Maxwell-Bloch equations in the rotating frame are accordingly,

$\frac{\partial}{\partial z} x_{L}(z, t)=-\frac{g^{*}(z) \Omega(t)}{\sqrt{2} \Delta}\left(\cos \left(\omega_{L} t\right) p_{A}(z)+\sin \left(\omega_{L} t\right) x_{A}(z)\right)$,

$\frac{\partial}{\partial z} p_{L}(z, t)=0$

$\frac{\partial}{\partial t} x_{A}(z, t)=-\frac{g^{*}(z) \Omega(t)}{\sqrt{2} \Delta} \cos \left(\omega_{L} t\right) p_{L}(z, t)$,

$\frac{\partial}{\partial t} p_{A}(z, t)=\frac{g^{*}(z) \Omega(t)}{\sqrt{2} \Delta} \sin \left(\omega_{L} t\right) p_{L}(z, t)$.

Now the atomic momentum operator, i.e. the spin projection along the axis of light propagation, is not conserved anymore and the overall interaction is not of QND character. Integration of these equations becomes somewhat more involved than before. We will resort to this problem in sections $\mathrm{IV}$ and $\mathrm{VI}$.

\section{F. Quantum measurement and feedback}

In this section we will deal with measurements which can be done on light and feedback on atoms. We will focus on homodyne detection of light, which is of importance for experiments using Faraday interaction and EIT and also briefly describe photon counting, which is used in combination with parametric gain and beam splitter interactions.

Homodyne detection of light The discussion of the quantum interface in the language of canonical variables for light is most fruitful because these variables can be measured with almost perfect efficiency by the balanced homodyne technique. We will concentrate here on the polarization homodyne version which is relevant for several protocols described in the article. In particular in the context of the Faraday interaction the polarimetric measurement of light is an important tool. Balanced homodyning employs overlapping the quantum field of interest with a strong coherent field, a local oscillator, on a 50/50 beamsplitter and measurement of the difference of the power in the two outputs. In its polarization version as shown in Fig. 5 the local oscillator field and the quantum field are overlapped on a polarizing cube so that they have orthogonal polarizations and the role of the beamsplitter is played by a polarizing beamsplitter which splits the light into $45^{0}$ and $-45^{0}$ modes. The measurement of the differential power of these two modes corresponds to the measurement of the $S_{y}$ Stokes operator, whereas with an extra $\lambda / 4$ plate in front of the beamslitter the $S_{z}$ Stokes operator is measured:

$$
\begin{aligned}
S_{x}(t) & =\left(a_{L, x}^{\dagger} a_{L, x}-a_{L, y}^{\dagger} a_{L, y}\right) / 2, \\
S_{y}(t) & =\left(a_{L,+45^{\circ}}^{\dagger} a_{L,+45^{\circ}}-a_{L,-45^{\circ}}^{\dagger} a_{L,-45^{\circ}}\right) / 2 \\
& =\left(a_{L, x}^{\dagger} a_{L, y}+a_{L, y}^{\dagger} a_{L, x}\right) / 2, \\
S_{z}(t) & =\left(a_{L, \sigma^{+}}^{\dagger} a_{L, \sigma^{+}}-a_{L, \sigma^{-}}^{\dagger} a_{L, \sigma^{-}}\right) / 2 \\
& =-i\left(a_{L, x}^{\dagger} a_{L, y}-a_{L, y}^{\dagger} a_{L, x}\right) / 2 .
\end{aligned}
$$

The third Stokes operator $S_{x}$ is equal to the total photon number of the strong field for the case of the strong coherent field $|\alpha\rangle$ in linear $x$ polarization, such that $\left\langle S_{x}(t)\right\rangle=|\alpha|^{2} / 2$. In this case the measurement of the two other Stokes operators amounts to a homodyne detection of $y$ polarized light with the coherent field in $x$ serving as the local oscillator,

$$
\begin{aligned}
& \frac{S_{y}(t)}{\sqrt{\left\langle S_{x}\right\rangle}} \simeq \frac{1}{\sqrt{2}}\left(a_{L, y}(t)+a_{L, y}^{\dagger}(t)\right)=x_{L}(t), \\
& \frac{S_{z}(t)}{\sqrt{\left\langle S_{x}\right\rangle}} \simeq-\frac{i}{\sqrt{2}}\left(a_{L, y}(t)-a_{L, y}^{\dagger}(t)\right)=p_{L}(t) .
\end{aligned}
$$

The homodyne detection can also provide an excellent suppression of the technical (classical) noise if the frequency of the local oscillator and the quantum field differ 


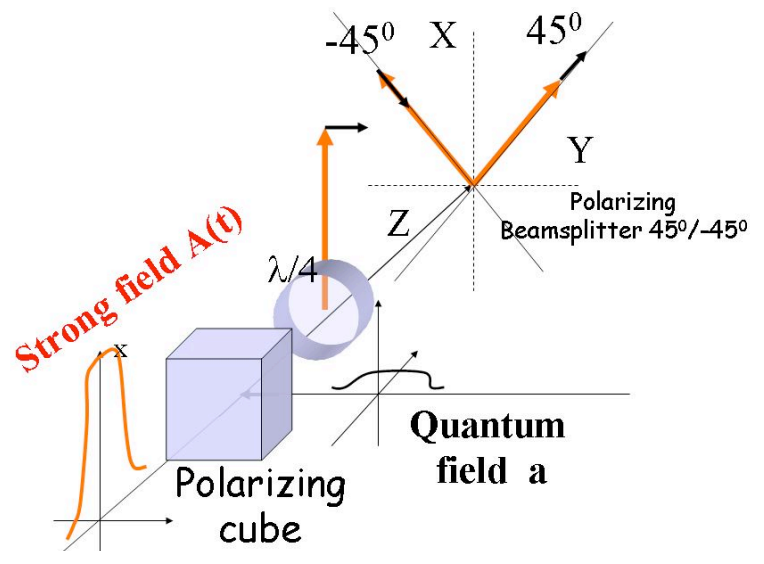

FIG. 5 Polarimetric measurement of light

by $\omega_{L}$ lying in the radio frequency domain, see Fig. 4 . In this case the relevant canonical variables are encoded in sideband modulation modes of $y$-polarized light which are read in the $\cos \left(\omega_{L} t\right)$ and $\sin \left(\omega_{L} t\right)$ components of the photodetector output:

$$
\begin{aligned}
X_{L_{c}} & =\sqrt{\frac{2}{T}} \int d t \cos \left(\omega_{L} t\right) x_{L}(t), \\
P_{L_{c}} & =\sqrt{\frac{2}{T}} \int d t \cos \left(\omega_{L} t\right) p_{L}(t), \\
X_{L_{s}} & =\sqrt{\frac{2}{T}} \int d t \sin \left(\omega_{L} t\right) x_{L}(t), \\
P_{L_{s}} & =\sqrt{\frac{2}{T}} \int d t \sin \left(\omega_{L} t\right) p_{L}(t) .
\end{aligned}
$$

These components of the photocurrent can be measured by lock-in amplifiers. The bandwidth of this measurement can be adjusted to $B W \approx \tau^{-1}$ where $\tau$ is the optical pulse duration. In this way fluctuations at all frequencies outside this bandwidth are effectively irrelevant. In the case where atomic ground state levels are non-degenerate, e.g., are split by an external magnetic field by the Larmor frequency $\omega_{L}$ as discussed in Sec. II.E and shown in Fig. 4, the atoms couple to the sidebands of light and the entire measurement and interaction can be encoded at sideband frequencies $\pm \omega_{L}$, as in several experiments described later in the article.

Feedback Another important tool in many quantum information protocols is feedback of results of measurement of light onto atoms. The theory of quantum feedback is a wide field on its own, especially in the case of continuous measurement and feedback (Thomsen et al. 2002a). Here we deal with a relatively simple measurement and feedback scheme, where light observables of the type (66) are measured by integrating a photocurrent over the whole pulse duration and the measurement result, a single number, is fed onto the atoms. The operations which need to be done on the collective spins are small rotations about the $y$ or $z$ axis, i.e. small tilts of the collective spin. In the language of canonical operators $X_{A}, P_{A}$ this amounts to displacements in the phase plane (Arecchi et al. 1972).

In this case - feedback of integrated measurement results via displacement operations - a simple rule can be applied for describing the overall effect on a state of the atoms. Assume the state of the system is described by certain input-output relations of the type (40). If a quadrature of light, say $X_{L}$, is measured and the corresponding measurement result $\xi$ is used to displace the atomic state, i.e. to tilt the collective atomic spin, in such a way that the mean of, say in $P_{A}$, is transformed as $\left\langle P_{A}\right\rangle \rightarrow\left\langle P_{A}\right\rangle+g \xi$, then the statistics of $P_{A}$ after the feedback operation can be calculated from

$$
P_{A, \text { final }}=P_{A}+g X_{L},
$$

that is, one simply needs to add the measured observable multiplied by the gain to the operator which is subject to the feedback. This rule for describing the feedback holds strictly as an operator identity, irrespectively of the state of the system being Gaussian or Non-Gaussian.

The proof is most easy in the Schrödinger picture. Assume the state of some bipartite system is $\hat{\rho}_{A L}$, where the indices refer, e.g., to atoms and light respectively. A measurement of $X_{L}$ gives a result $\xi$ with probability $p_{\xi}=\left\langle\xi\left|\operatorname{tr}_{A}\left\{\rho_{A L}\right\}\right| \xi\right\rangle$, where $X_{L}|\xi\rangle=\xi|\xi\rangle$, and the state of the system $A$ collapses to

$$
\rho_{A}^{(1)}=p_{\xi}^{-1}\left\langle\xi\left|\rho_{A L}\right| \xi\right\rangle .
$$

The feedback affecting the desired displacement is described by a unitary transformation of the state of the system $A$,

$$
\rho_{A}^{(2)}=e^{i g \xi X_{A}} \rho_{A}^{(1)} e^{-i g \xi X_{A}},
$$

which gives in the ensemble average over all possible measurement results the final state

$$
\begin{aligned}
\rho_{A}^{(3)} & =\int d \xi p_{\xi} \rho_{A}^{(2)}=\int d \xi e^{i g \xi X_{A}}\left\langle\xi\left|\rho_{A L}\right| \xi\right\rangle e^{-i g \xi X_{A}} \\
& =\int d \xi\left\langle\xi\left|e^{i g X_{L} X_{A}} \rho_{A L} e^{-i g X_{L} X_{A}}\right| \xi\right\rangle \\
& =\operatorname{tr}_{L}\left\{e^{i g X_{L} X_{A}} \rho_{A L} e^{-i g X_{L} X_{A}}\right\} .
\end{aligned}
$$

From this equation all the moments of $P_{A}$ can be calculated as

$$
\begin{aligned}
\left\langle P_{A}^{n}\right\rangle & =\operatorname{tr}_{A}\left\{P_{A}^{n} \rho_{A}^{(3)}\right\} \\
& =\operatorname{tr}_{A L}\left\{\left(P_{A}+g X_{L}\right)^{n} \rho_{A L}\right\},
\end{aligned}
$$

where the cyclicity of the trace was used in the second equality. This justifies the rule given above.

Note that for Gaussian states, for which it is enough to keep track only of the first and the second moments in order to have a full knowledge of the state, the simple linear transformation of operators as above is exactly equivalent 
to a full description in the Schrödinger picture e.g. on the basis of the Wigner function. The measurement of and the feedback on more than one mode can be described by an immediate generalization of (67) as shown in (Hammerer et al., 2005a, Sherson et al., 2006c), provided the measurement involves commuting observables only.

Photon Counting In some cases measurements in the Fock state basis, i.e., photon counting are convenient for characterization of the interface performance. As discussed in Sec. V, storage and retrieval of some nonclassical states can be characterized by the measurement of the second order correlation function $g^{2}(1,2)$ which is a normalized probability of photon counts at two points in space-time (Loudon, 2004). Atoms typically used for the interface are Rubidium and Caesium and the corresponding spectral lines are around 780nm and 850nm respectively. In this spectral domain commercial avalanche photodiodes (APDs) typically have quantum efficiency around $40-50 \%$ and the dark count rate of a few hundred per second. Such parameters are sufficient to determine non-classical correlations via $g^{2}(1,2)$ which are only weakly sensitive to imperfect quantum efficiency.

\section{G. Other Strategies}

In the discussion so far we have focussed on what we consider to be the main protocols in this field. There are, however, numerous variations of all of these protocols, some of which we will briefly discuss here.

Non-copropagating beams. In the derivation above we have only considered the situation, where the quantum and classical light fields are co-propagating. For many applications of the beam splitter and parametric gain interaction this assumption is, however, not necessary (Balić et al. 2005, Braje et al., 2004, Chaneliere et al. 2005). For instance, an incoming photon which is absorbed in an atomic ensemble by using a co-propagating classical field generates an excitation of the form of Eq. (18). The reason why one can later retrieve this quantum state is constructive interference. During readout all atoms will radiate in phase in the direction of the classical laser and this is the effect, which allows for efficient interfaces between atoms and light in the limit of a large number of atoms (high $d$ ). If the photon which is absorbed has a different direction than the classical drive field, the generated atomic excitation will still have the form of Eq. (18). The only difference is that the state will have a phase factor $\exp \left(i \Delta \vec{k} \cdot \vec{r}_{i}\right)$ on the component, where the $i$ th atom is in state $|1\rangle$, with $\Delta \vec{k}$ being the difference between the $k$-vectors for the two fields. In order to have constructive interference in the readout process the difference in the $k$-vectors of the outgoing photon and the classical field in the read out process should exactly cancel the phase factor imprinted on the atoms in the first step of the protocol.

Expressed in different terms, the initial atomic state $|000 \ldots . .0\rangle$ has a homogeneous phase corresponding to a zero momentum state. The initial process involving the absorption of a photon from one beam and the emission of a photon into a different beam imprints the difference momentum $\Delta \vec{k}$ onto the atomic spin wave. Constructive interference is achieved for processes returning the atoms to the initial zero-momentum spin wave $|000 \ldots . .0\rangle$ and the difference momentum in the read out process must therefore carry away the momentum in the spin wave. To achieve momentum conservation (or equivalently phase matching) the total momentum of all the absorbed photons should thus match the total momentum of all the emitted photons.

A disadvantage of using non co-propagating beams is that the storage into non-symmetric modes limits the storage to the time it takes atoms to move a distance $\sim 1 /|\Delta \vec{k}|$. In particular for room temperature gasses (see Sec. III.A this, as well as the differential Doppler-shift, makes it undesirable to use geometries, where the beams are not nearly co-propagating. On the other hand it is often a major experimental advantage not to have the classical and quantum beams co-propagating since this makes it much easier to count photons in the quantum beam.

Interaction based on phase shift It is instructive to have a look at the Faraday interaction from another perspective. Consider first Fig. 6(a) where, as before, the quantization axis is taken along $x$, the direction of polarization of both atoms and light. Selection rules for dipole transitions dictate that strong classical, $x$-polarized light with amplitude $a_{x} \simeq \alpha$ drives the $| \pm\rangle_{x} \rightarrow| \pm\rangle_{x}$ transitions, while the $| \pm\rangle_{x} \rightarrow|\mp\rangle_{x}$ cross transitions are coupled to the weak quantum field $a_{y}$ in $y$-polarization. In this picture it is evident that the Faraday interaction is the sum of the beam splitter and the parametric gain interaction, cf. Fig. 2 The same level configuration can also be looked at by taking the axis of quantization along the $z$ direction, as shown in Fig. 6(b), where $| \pm\rangle_{z}=\left(|+\rangle_{x} \pm|-\rangle_{x}\right) / \sqrt{2}$. Light propagating along this direction naturally couples with its circular components $a_{\sigma^{ \pm}}=\left(a_{x} \pm i a_{y}\right) / \sqrt{2} \simeq\left(\alpha \pm i a_{y}\right) / \sqrt{2}$ to the cross transitions $| \pm\rangle_{z} \rightarrow|\mp\rangle_{z}$ only. The off-resonant coupling will thus give rise to $\mathrm{AC}$ Stark shifts on atomic levels $| \pm\rangle_{z}$ depending on light intensities of $\sigma^{ \pm}$-polarization components. Vice versa, light polarization is rotated according to the difference in level populations $| \pm\rangle_{z}$.

From this observation it is clear, that all that is required for a Faraday interaction is a mechanism of nondestructive measurement of level populations via phase shifts of light. Making use of the vector polarizability for probing the collective hyperfine spin-angular momentum as described above, is therefore just one way to achieve a Faraday or QND interaction. In addition, several propos- 
(a)

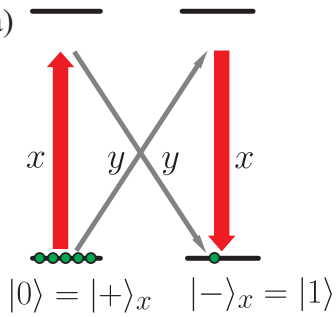

(b)

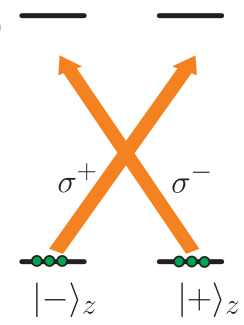

FIG. 6 Level scheme for Faraday interaction: (a) For axis of quantization along $x$ : Atoms are polarized to $|+\rangle_{x}$, laser light is linear polarized along $x$ and drives the up-transitions and the quantum field in $y$ polarization couples to the crosstransitions. (b) The same interaction with axis of quantization taken along $z$ : Circular light components of equal intensity cause AC Stark shifts of equally populated states $| \pm\rangle_{z}$.

als and experiments pursue the idea to probe coherences of the pseudo-spin consisting of the two $S_{1 / 2}(F=I \pm 1 / 2)$ hyperfine levels, see (Chaudhury et al. 2006: Oblak et al. 2005: Petrov et al. 2007, Windpassinger et al. 2008). As shown in Fig. 7 at certain detuning the phase shift of the probe light due to $F=I \pm 1 / 2 \rightarrow F^{\prime}$ transitions exactly cancel for equal populations of $F=I \pm 1 / 2$ levels. Any imbalance of populations will yield an interferometrically detectable phase shift.

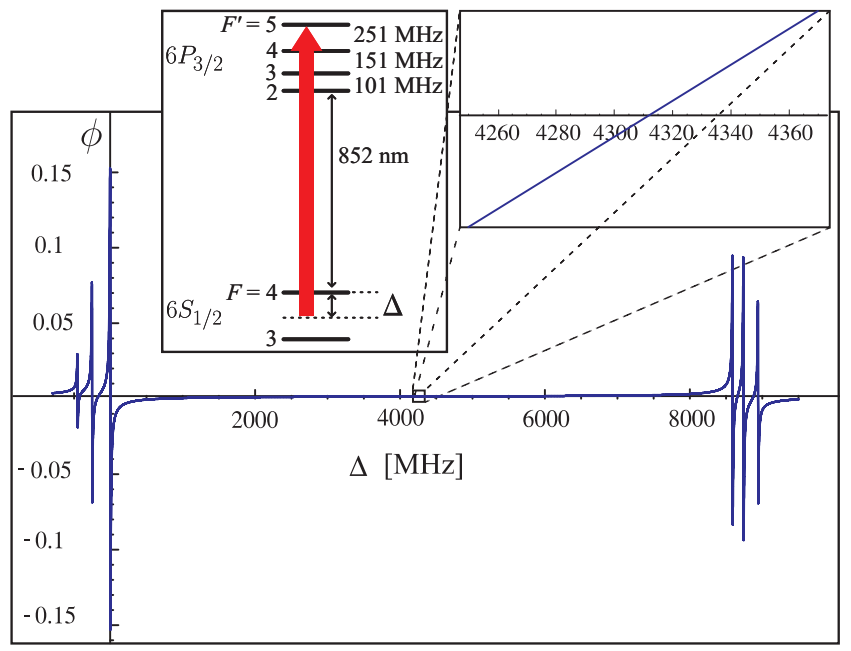

FIG. 7 (a) Level scheme for ${ }^{1} 33$ Cs with probe light tuned in between hyperfine levels $F=3,4$ of the $6^{2} S_{1 / 2}$ ground state. (b) Differential phase shift $\Phi$ due to the $F=3 \rightarrow F^{\prime}=2,3,4$ and the $F=4 \rightarrow F^{\prime}=3,4,5$. At magic frequencies the phase shift vanishes.

Other Hamiltonians and level structures. There are several possibilities involving more complicated atomic level structures than the ones shown in Fig. 2 A particular example is the so called double $\Lambda$-systems, with two excited states. An interesting feature of this system is its potential application for four wave mixing. For a review of this see Fleischhauer et al. (2005).
In the derivation of the theory we adiabatically eliminated the excited state to arrive at the effective ground state Hamiltonian. As discussed in Subsec. [I.D this adiabatic elimination in general leads to a Hamiltonian involving spherical tensors of rank zero, one, and two with strength characterized by the three coefficients $a_{0}, a_{1}$ and $a_{2}$. The three protocols that we have mainly considered thus correspond to suitable initial states and particular combinations of these spherical tensors. By adjusting the detuning as well as laser polarizations and atomic initial state there is, however, a lot of freedom in varying the relative strength and effect of the different tensors, which allow for a richer dynamics. Kupriyanov et al. (2005) and Mishina et al. (2007) considered how the higher order tensor operators modify the equations of motion and in particular how the Faraday interaction is influenced by the rank two tensor. For instance in Mishina et al. (2007) it was shown that a particular choice of detuning removes the AC-Stark for a detuned beam splitter interaction and thus removes the need to adjust the frequency of the classical driving field in order to keep the field in two photon resonance with the AC-Stark shifted transition. To arrive at the Faraday interaction we just combined the beam splitter and parametric gain interaction with the same strength, but in Mishina et al. (2006) it was shown that an arbitrary combination of the parametric gain and beamsplitter interaction can be obtained by choosing suitable initial conditions and combinations of the spherical tensor.

An example of a protocol where the light-atom interface involves excited states without adiabatic elimination is shown in Fig. 1(a) (Hald et al., 1999, Kuzmich et al., 1997). Another protocol of this type is considered in Sec. V.E. where we discuss spin echo techniques. A disadvantage of such protocols is, however, that the storage time is limited by the coherence time of the optically excited state, which is often shorter than the coherence time of ground states.

Optical cavities. The key parameter in characterizing the applicability of an atomic ensemble for a light matter quantum interface is its optical depth which for a free space ensemble is limited by the size and atomic interaction. An alternative strategy is to use multiple passes of the light through the atomic ensemble by enclosing the ensemble in an optical cavity (Black et al., 2005, Dantan et al., 2005; Josse et al., 2004; Simon et al., 2007a b; Thompson et al. | 2006). In this case the parameter characterizing the usefulness of the system is the cooperativity parameter $C=N_{A} g_{c}^{2} / \kappa_{c} \gamma$, where $g_{c}$ is the coupling constant for a single atom to the cavity mode, and $\kappa_{c}$ is the cavity decay rate. The cooperativity can also be expressed as $C \sim \mathcal{F} d$, where $\mathcal{F}$ is the finesse of the cavity which roughly equals the number of passes that the photon makes through the cavity (Gorshkov et al., 2007b). The gain by using a cavity thus equals the number of round trips. 
Non Gaussian operations. In this review we consider the quantized light fields which are much weaker than classical control and driving light, and the quantum fluctuations of the atomic ensemble which are much smaller than the mean spin. In this limit we only include the lowest order terms in the atomic and light field operators $a_{A}$ and $a_{L}$. Because there are no first order terms the effective Hamiltonian will be quadratic in the harmonic oscillator operators. The operations which may be performed thus fall into the class of Gaussian operations and the solution of the equations will in general be a Bogoliubov transformation of the incident mode operators Braunstein and van Loock, 2005). For any input state with a Gaussian Wigner function the output Wigner function will also be Gaussian. The main advantage of using atomic ensembles is that the dynamics resulting from these Gaussian operation are collectively enhanced so that a perfect operation is achieved in the limit of large optical depth. While the resulting dynamics allow for a variety of quantum information protocols to be performed, such as quantum teleportation and quantum memory ( $\mathrm{sec} \mathrm{V}$ and $\mathrm{VI}$, the fact that higher order terms are not collectively enhanced limits the applications for quantum information processing. In particular it is known that Gaussian operations alone do not allow for distillation of entanglement from Gaussian states (Eisert et al. 2002 , Fiurǎśek, 2002; Giedke and Cirac, 2002) and there exists algorithms for efficient classical simulation of any evolution involving only Gaussian operation and Gaussian initial states (Bartlett et al. 2002 , Lloyd and Braunstein, 1999). These limitations may, however, be avoided by combining the Gaussian operation with photon counting Genes and Berman, 2006, Neergaard-Nielsen et al. 2006, Ourjoumtsev et al. 2006). In the pioneering paper by Duan et al. (2001) such photon counting techniques were proposed as a means for quantum communication over long distances using the probabilistic entanglement protocols discussed in Sec IV.C Such techniques could in principle also allow for even more advanced quantum information protocols to be implemented.

The fundamental obstacle for directly achieving non Gaussian operations is that they rely on an interaction between individual excitations. Such interactions between the excitations can for instance be achieved if two photons interact with the same atom. But since this is essentially a single atom effect it is not enhanced by a large optical depth. An approach which allows for an enhancement of this nonlinear effect by optically imprinting a Bragg mirror which localizes excitation similar to an optical cavity is explored in Bajcsy et al. (2003) and André and Lukin (2002). An alternative approach to non Gaussian operations is to engineer a strong interaction between excitations stored in different atoms. Interesting proposals in this direction are to use the collisional interactions of atoms in optical lattices (Muschik et al. 2008) or the so called Rydberg blockade, where the excitation of a single atom to a Rydberg level blocks the excitation of other atoms, and therefore creates a uni- form long distance interaction (Lukin et al., 2001). Alternatively one can exploit the fact that atomic ensembles are particularly well suited for "catching" traveling photons and then afterwards transfer the excitation to some other system for processing the information. A proposal along these lines is presented by Rabl et al. (2006) based on a transfer of excitations from an ensemble of dipolar molecules to a solid state system. A review of techniques for achieving other types of operations, e.g., Kerr interactions, is given in Fleischhauer et al. (2005).

\section{H. Summary of the theory}

Above we have presented a detailed theory for the quantum interfaces between light and atomic ensembles. In particular we have presented a unified theory for the three model systems presented in Fig. 2(a)-(c), the beam splitter, the parametric gain, and the QND (Faraday) interaction. The three systems have distinct features, but are also interconnected, e.g., the Faraday interaction is just a combination of the beam splitter and parametric gain interaction. Most importantly all three systems achieve ideal operation in the limit of high optical depth $d$. This feature can be understood as constructive interference or collective enhancement of the coupling: the coupling between the state where all atoms are in the ground state and the collective state 18 scales as $\sqrt{N_{A}}$, whereas spontaneous emission is a single atom effect, which is independent of the atom number.

In the far off-resonant limit $\Delta \gg \gamma$ the strength of the coupling is parametrized by exactly the same function $h(0, T) \approx \kappa^{2}$, cf. Eqs. 48 and (57), which is most easily seen by rewriting the equations in the dimensionless form as in Appendix IX.E. In this limit the decay of the light can be ignored and the coupling constant can be related to $\eta_{A}$, the spontaneous emission probability per atom, through $\kappa^{2}=\eta_{A} d$, which we explicitly derived for the Faraday interaction, but which also applies to the other systems. From this expression one thus directly sees that the spontaneous emission can be eliminated for high optical depth. In a special case of the beam splitter interaction, the resonant EIT setting, these arguments are not directly applicable, but the ratio between the desired evolution and spontaneous emission, i.e. the constructive interference discussed above, is completely independent of detuning (Gorshkov et al., 2007a c).

It is instructive to discuss the bandwidth of the quantum interface. In the theory we have adiabatically eliminated the excited state, which means that the bandwidth is limited by the low saturation condition $s \ll 1$ and the condition on the value of the coupling constant $\kappa$ necessary for a particular process. Bearing in mind the relations $\eta_{A}=\gamma \tau s, \tau=(B W)^{-1}$, we can draw some general conclusions on the Fourier limited bandwidth $B W$ of the process. For protocols where $\kappa^{2} \sim 1$ the low saturation condition yields the limitation on the bandwidth of the light pulse, $B W \ll \gamma d$ (again the arguments given here 
only directly apply in the far off resonant limit, but the conclusion is also valid for EIT). Going beyond the low saturation regime $s \sim 1$ allows to increase the bandwidth somewhat (Gorshkov et al., 2008), but it remains limited by $B W \lesssim \gamma d$. For a typical alkali atomic ensemble the bandwidth of the order of $10 \mathrm{MHz}$ can be achieved. This scaling of the BW provides an upper limit, e.g., for an atomic EPR entanglement protocol discussed later in the article where the conditions $\kappa^{2} \gg 1, \eta \approx 1 / \sqrt{d}$ has to be met, the limit on the bandwidth is $B W \ll \gamma \sqrt{d}$.

\section{ATOMIC MEDIA FOR QUANTUM INTERFACE}

A few common requirements can be formulated for all ensemble based interfaces described in the previous sections. A long lived (ground) state of atoms is commonly used. This could, e.g., be Zeeman levels or hyperfine levels. The ensemble should be initialized to a polarized state (coherent spin state), that is one of the ground substates should be populated by optical pumping or other means. Most importantly, the sample should have a large resonant optical depth $d$. Up to date, experimental realizations of the ensemble based interfaces utilize alkali atom gases at room temperature, alkali atoms cooled and trapped at temperatures of a few tens or hundreds of microkelvin, or impurity centers in solid state. Below we describe these and other media used for quantum interfaces.

\section{A. Room temperature gases}

A gas sample of alkali atoms is one of the simplest atomic ensembles to have in the lab. Surprisingly enough such an object can also work very well as a quantum memory, if proper care of decoherence is taken. The thermal motion and associated with it Doppler broadening are not necessarily a problem. For the Faraday interaction, the Doppler broadening plays little role if the detuning is much greater than the Doppler width (200-300 $\mathrm{MHz}$ for Cesium or Rubidium). For other protocols such as the beam splitter interaction the Doppler broadening has a detrimental but still tolerable effect as discussed in Sec. VII

In addition to the Doppler broadening the atomic motion also leads to changes in the atomic positions. A quick glance at the solution to the beam splitter interaction (47) and (50) reveals that the atomic operators with different longitudinal coordinates experience different dynamics. The atomic motion in the process of interaction leads to washing out of these spatial modes which is a much more pronounced problem for the beam splitter interaction, as compared to the Faraday interaction.

In order to reduce the deleterious effect of atomic motion a buffer gas is usually used in the experiments which utilize the beam splitter interaction in gas cells at room temperature (Eisaman et al. 2005; Novikova et al., 2007).
Adding a few torr of a noble gas allows to sufficiently localize the diffusive motion of alkali atoms. An extra benefit of this approach is that it prevents alkali atoms from depolarizing collisions with each other and with the walls of the cell which otherwise could lead to a rapid decoherence. The lifetime of the atomic memory in cells with a buffer gas can reach milliseconds (Novikova et al., 2007). Note that for some protocols, collisions of atoms in the excited state with the buffer gas lead to an energy redistribution of scattered photons which may lead to large errors in the absence of careful spectral filtering (Manz et al. 2007). Despite the difficulties, alkali atom cells with a buffer gas has been successfully used for experiments on quantum memory using EIT (Eisaman et al. 2005).

The effect of atomic motion for the Faraday interaction can be almost completely eliminated. As follows from the propagations equations 40 the Faraday interaction couples light to a symmetric atomic mode defined in Eq. 16. In this case atoms with different coordinates $z$ along the direction of light propagation couple to light in the same way. Hence the atomic motion along $z$ does not affect the interaction. The transverse motion of atoms along $x$ and $y$ axes will affect the performance if the spatial profile of the light beam is inhomogeneous which is almost always the case. This effect can be reduced in two extreme cases, either for times short compared to the motion time, or in case when the duration of the light pulse is so long that atoms have a chance to cross the beam many times during the interaction and the effect of motion averages out. The latter was the case of the experiments by Julsgaard et al. (2001, 2004a) and Sherson et al. (2006a), where pulses of about $1 \mathrm{msec}$ duration have been used.

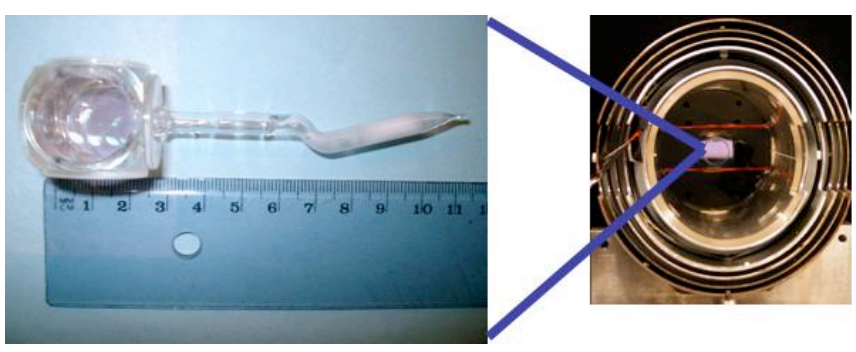

FIG. 8 Paraffin coated Caesium cell.

The possibility to eliminate the effect of atomic motion on the efficiency of the interface based on the Faraday interaction has allowed to conduct high-fidelity experiments with room temperature Cesium atoms (Julsgaard et al. 2001, 2004a; Sherson et al., 2006a). Atoms were contained in cells with a paraffin coating of the internal walls (Fig. 8). Such coating has been used in precision magnetometers for the past three decades Alexandrov, 2003, Groeger et al., 2006), and ground state coherence times of up to a second have been demonstrated. In paraffin coated cells atoms can withstand tens of thousands collisions with the cell walls before significant spin 
depolarization occurs. Since it is the number of collisions with walls that matters, the larger is the cell, the longer is the quantum memory lifetime. As discussed in details in Sections IV, V, and VI, quantum memory time of the order of several milliseconds has been achieved in cells with dimensions $25 \times 25 \times 25 \mathrm{~mm}^{3}$.

Room temperature ensembles of Cesium atoms of a few cubic $\mathrm{cm}$ size contain about $10^{12}$ atoms. For Faradaytype interfaces the Doppler broadening does not affect the ensemble effective resonant optical depth (see sec. VII] which is the same as for atoms at rest reaching the values of the order 50 or even higher. The experimental challenge lies with the fact that the quantum spin noise of such an ensemble is ${\sqrt{N_{A}}}^{-1} \approx 10^{-6}$. In order to reach the level of the spin quantum noise, all types of technical spin fluctuations, such as driven by stray magnetic fields or fluctuations of the lasers used for optical pumping, have to be reduced below this level. The solution to this problem used in Julsgaard et al. (2001. 2004a); Sherson et al. (2006a) has been to apply a bias magnetic field along the direction of the collective atomic spin. A field of the order of one Gauss provides the Zeeman splitting of the ground state $\omega_{L}$ of a few hundred $\mathrm{kHz}$. As discussed in Sec. II.Ethis means that the collective transverse components of the spin which correspond to the atomic canonical variables rotate at the Zeeman frequency. At the same time, as described in Sec. II.F. canonical variables of light which couple to the rotating atomic spin can be measured via homodyne detection also at the Zeeman frequency. Thus all relevant variables for light and atoms are now encoded at a frequency of a few hundred $\mathrm{kHz}$. At these frequencies technical noise can be reduced below the $10^{-6}$ level, so that both spin and light fluctuations are dominated by quantum noise. In practice the photocurrent detected in the homodyning process is measured with lock-in amplifiers which allow access to the light variables (66) encoded at the frequency $\omega_{L}$. In the experiments by Julsgaard et al. (2001, 2004a); Sherson et al. (2006a) the bandwidth of the memory has been reduced to around $1 \mathrm{kHz}$ for the reasons discussed in Sec. II.H.

\section{B. Cold and trapped atoms}

Cold and trapped ensembles of alkali atoms have been among the first atomic objects to be used for quantum interfaces with light. The first experiment mapping quantum properties of light onto atoms was performed with Caesium atoms in a MOT, a magneto-optical trap (Hald et al. 1999). A MOT provides a relatively simple way to achieve a cold atom sample suitable for the quantum interface, however it also has its limitations. A typical resonant optical depth in a MOT lies in the range between 2 and 10 which is not very high. Another consideration concerns the transverse crossection of the light beam which couples to the atoms. In most of the experiments on interfaces which use a MOT, light is focused down to a few tens of microns which is much less than a typical MOT crossection of a millimeter Chaneliere et al., 2005, Chen et al., 2008; Chou et al., 2004, Dantan et al. 2005: Simon et al., 2007b). Such geometry limits the atomic memory lifetime to the transient time it takes the atoms to leave the probe volume. For a typical MOT temperature of $100 \mu \mathrm{K}$ this time is around tens of microseconds. If, on the other hand, the beam crossection is such that the light couples to the entire MOT the transient effects become irrelevant. However, in this case the number of photons in the strong driving field $N_{P}$ grows proportionally to the cross section $A$, as evident from Eq. 61). When a photon number of the strong field is too high it becomes more difficult to implement protocols based on separating and photon counting of the quantum mode. Protocols based on homodyne detection also place limits on the maximal number of photons in the driving field. In most cases the driving field is also used as the local oscillator for homodyne detection. This implies that in order for the detection to be shot noise limited the classical fluctuations of the strong field should be suppressed to better than $N_{P}^{-1 / 2}$. In practice this places the limit $N_{P} \leq 10^{10}$. For higher values of $N_{P}$ modulation techniques similar to those used with thermal ensembles allow to get down to shot noise limited detection.

Another difficulty of working with a MOT is due to the presence of gradient magnetic fields and the associated difficulty of optical pumping and magnetic states decoherence. This problem can be overcome by switching off the MOT fields which in turn limits the lifetime of the interface. An advantage of using a small sub-ensemble of a large MOT is that a single MOT can then serve as a source of two or more atomic ensembles (Choi et al. 2008: Matsukevich and Kuzmich, 2004).

Using a far detuned dipole trap allows to overcome a number of problems associated with the MOT. A dipole trap forms an atomic ensemble with a typical transverse size of around $10 \mu \mathrm{m}$ which is a good size for the interface. The resonant optical depth can reach 20 or more. Dipole trapped atomic ensembles demonstrate coherence times exceeding 10 msec (Windpassinger et al. 2008). Dipole trapping can also be insensitive to the magnetic quantum number and, in some cases, to the hyperfine quantum number. A detailed investigation of dipole trapped atoms as the medium for the spin squeezing and the interface is given in (Oblak et al. 2005; Windpassinger et al., 2008).

A Bose-Einstein condensate (BEC) is an attractive medium for the quantum interface due to its very high resonant optical depth. Indeed, BEC has been the medium where classical coherent storage of light, the so called stopped light has been first demonstrated (Liu et al. 2001). It is important to note that it is not only the on-axis optical depth that defines the strength of the interface coupling. For example, a "one dimensional" sample will not couple efficiently to a focused Gaussian beam because the diffraction of the beam means that it will not be overlapping with the ensemble if the Fresnel number is less than unity (Müller et al. 2005). 
One problem with a BEC-based quantum interface is the low rate at which experiments can be performed. A typical BEC requires tens of seconds to be created. Then the sample can be used for interface experiments a few times, after which a new sample should be created. BEC on a chip (Hansel et al. 2001; Schneider et al., 2003) offers an attractive alternative where much faster loading times can be combined with very efficient optical coupling.

\section{Solid state}

An optically dense collection of atom-like impurities in a solid state host is an excellent candidate for the quantum interface. Both $\Lambda$ schemes as well as the photon echo-based memory (see Sec. V.E have been investigated. The absence of motion in solid state means that complex spatial structures can be generated by light and stored. Recently substantial progress has been achieved with crystals or glasses doped with rare-earth elements, such as erbium (Er), thulium (Tm), praseodymium (Pr), and europium (Eu). The rare-earth ions doped into glass and crystal materials (REIC) display up to a second coherence times of the ground state at liquid Helium temperatures. The ions experience strong inhomogeneous broadening up to $100 \mathrm{GHz}$ due to local lattice fields. The technique of spectral hole burning (Nilsson et al., 2004 ) followed by spectrally selective anti-hole populating allows to create a sub-ensemble of ions with nearly natural optical transition bandwidth. A substantial optical depth can be created, although its value is usually limited by the ion-ion interaction at high density of doping. Note, however, that one cannot directly compare the measured optical depth in the presence of inhomogeneous broadening with the optical depth introduced in the theoretical derivation in Sec. II, see sec. IVII. EIT- and Ramanbased memory (Longdell et al., 2005) has been explored in REIC materials as well as a photon echo approach based on Controlled Reversible Inhomogeneous Broadening (CRIB) (Hétet et al. 2008a; Kraus et al., 2006. Moiseev and Kröll, 2001; Staudt et al., 2007)

The optical Raman coupling to the nuclear spin coherence has been investigated for the past decades in REIC, mostly in praseodymium- or europium-doped crystals. These materials seem particularly suitable for the EITand Raman-based quantum memory protocols since they exhibit a hyperfine structure where a $\Lambda$-system can be found, together with long optical coherence lifetimes, and also long hyperfine coherence lifetimes (15ms for Eu:YSO and $550 \mu \mathrm{s}$, that can be extended up to $30 \mathrm{~s}$ by dynamic decoherence control techniques, for Pr:YSO (Fraval et al. 2005)). The absorption wavelength of these materials is in the domain of dye lasers (606 nm for Pr and 580nm for $\mathrm{Eu})$. In order to take advantage of their long optical coherence lifetimes, the dye laser sources must be stabilized down to less than $1 \mathrm{kHz}$. The absorption wavelength of Tm lies in the convenient range of diode lasers $(793 \mathrm{~nm})$.
It also exhibits long optical coherence lifetimes, similar to that of Pr. It has been recently shown that it is possible to build a $\Lambda$-system in thulium by applying a magnetic field in a very specific orientation (Louchet et al., 2007). In rare-earth ion-doped crystals, the transitions are not polarization-selective, so the only way to address them separately is to use a source whose bandwidth is smaller than the ground state sublevel splitting. In Pr and Eu the splittings are fixed (10 and $17 \mathrm{MHz}$ for Pr:YSO, 75 and $102 \mathrm{MHz}$ for Eu:YSO) whereas in Tm they can be adjusted with magnetic field $(36 \mathrm{MHz} / \mathrm{T}$ in $\mathrm{Tm} ; \mathrm{YAG})$. The hyperfine coherence lifetime of up to $300 \mathrm{~s}$ has been measured in Tm:YAG.

FIG. showing spectral hole burning and anti-hole population.

Er-doped materials are studied with the photon echo CRIB method (Staudt et al. , 2007). The prime interest to this ion is due to the optical wavelength in the telecom band $1.5 \mu \mathrm{m}$. The optical coherence lifetime of this material is very short (a few $\mu$ sec at most), but can be dramatically increased by applying a very intense magnetic field. The most promising results up to date has been achieved in Pr-doped crystals (Hétet et al. 2008a. Longdell et al., 2005).

Materials containing a high concentration of quantum dots may be interesting candidates for the ensemblebased interface. Experiments with spin polarized dots show sufficient ground state coherence times and possibility of optical pumping and quantum nondemolition coupling (Atature et al. 2007). However, up to now the work with ensembles of dots has not reached quantum limits probably due to an insufficient optical depth. A different solid state medium which can be used for a quantum interface is Nitrogen vacancies in diamond, where EIT has been observed by Hemmer et al. (2001).

\section{Other possible media}

Optical lattices have attracted a lot of attention lately due to the exciting possibilities for generation of entanglement by controlled atom-atom interaction (Mandel et al. , 2003). The lattices can also display high optical depth since structures of up to $100 \times 100 \times 100$ atoms spaced by half a micron can be created. A quantum interface with such a lattice would offer an exciting possibility to transfer entanglement from atoms to light and to combine the quantum information processing capabilities of lattices with the quantum networking provided by the quantum interface. First theoretical studies of quantum interfacing of light with lattices have recently appeared (Eckert et al., 2008, Muschik et al., 2008).

Another system where a collection of atoms can be efficiently interfaced with light is a large ion crystal in an ion trap. Very clean and large ion crystals have been created and first attempts towards achieving quantum coupling to light have been undertaken (Herskind et al. 2008). 


\section{ENTANGLEMENT OF ATOMIC ENSEMBLES}

In this chapter we describe generation of entangled states of two distant macroscopic objects. The first method, which is based on QND interaction, measurement and feedback, generates an EPR (two-mode squeezed) state of atomic spins. The second method, which relies on parametric gain and beam splitter interactions and single photon counting, creates Bell states in two collective spins.

van Enk et al. (2007) gave a useful classification of the various types of entanglement, which are generated in experiments. They distinguish a priori entanglement, which can be deterministically generated, a posteriori entanglement, which is generated probabilistically and destroyed when measured, and finally heralded entanglement, which is as well probabilistically generated, but success can be testified by measuring an auxiliary system, such that the entangled state is still available for use. Using post-selection of successful cases, all types of entanglement are in principle equally useful. When combining a large number of entangled states, e.g. via entanglement swapping in a quantum network, the overall success probability will be dramatically different for a posteriori entanglement as compared to heralded or a priori entanglement. This observation lies at the heart of the original quantum repeater protocol (Duan et al. 2001), which is based on entanglement of atomic ensembles heralded by detection of single photons, as will be described in Sec. IV.C. Our main focus in Sec. IV.B will be on the a priori entanglement achieved via a QND-Bell measurement and feedback on two ensembles. To introduce this method, we first explain how a single atomic ensemble can be prepared in a spin squeezed state by means of a QND interaction, homodyne detection of light and feedback on atoms. Note that in Sec. V.D we describe the memory experiment (Choi et al. 2008 ) which involves a heralded entanglement as an intermediate step.

\section{A. Spin-Squeezing in a Single Ensemble}

Spin squeezed states of atomic ensembles were first introduced by Kitagawa and Ueda (1993) in analogy to squeezed states of the radiation field and suggested by Wineland et al. $(1994,1992)$ to be of use for enhancing the sensitivity in atomic spectroscopy and Ramsey interferometry. Accordingly, Kitagawa and Ueda (1993) define the state of a collective spin $J$ to be squeezed if the variance of one spin component $J_{\perp}$ transverse to its mean polarization is smaller than the transverse variance corresponding to an atomic coherent (Bloch) state (Arecchi et al. 1972), that is a product state of fully polarized atoms. With this definition, a state is squeezed if $\xi_{S}=\Delta J_{\perp} / \sqrt{J / 2}<1$ and necessarily consists of correlated atoms. Wineland et al. (1994, 1992) on the other hand show that the figure of merit for the supression of quantum fluctuations, which ultimetely limit the sensitivity of atomic Ramsey interferometry, is $\xi_{R}=(2 J)^{1 / 2} \Delta J_{\perp} /|\langle\vec{J}\rangle|<1$ and provides an alternative, stronger definition of spin squeezing.

We will here follow yet another definition and refer to a spin state as squeezed if $\xi=\Delta J_{\perp} /(|\langle\vec{J}\rangle| / 2)^{1 / 2}<1$, which is stronger than the definition due to Kitagawa and Ueda but weaker than the one due to Wineland et al. because $\xi_{R} \geq \xi \geq \xi_{S}$ as can be easily seen. The conditions are the same for nearly fully polarized states $\langle\vec{J}\rangle \approx J$. If we take the mean polarization along $x$, and assume that the transverse component with minimal variance is along $z$, the definition of spin squeezing adopted here is

$$
\Delta P_{A}^{2}<\frac{1}{2},
$$

where we use the Gaussian approximation 16 . We use this definition, because it will immediately translate in an entanglement criterion for a bipartite state of two ensembles in Sec. IV.B.

Various ways to create squeezed states in ensembles of two level systems were proposed and require either direct interaction of spins (Andre et al. 2002, $\mathrm{Pu}$ and Meystre, 2000: Sørensen et al. 2001; Sørensen and Mølmer, 1999), mapping of squeezed light onto atoms (Appel et al. 2008 , Dantan et al., 2006a; Hald et al., 1999, Honda et al., 2008, Kuzmich et al. , 1997), multiple passes of light through atoms (Hammerer et al., 2004, Takeuchi et al., 2005) or a projective, Faraday interaction based QND measurement (Braginsky and Khalili, 1996), as proposed by Kuzmich et al. (1998, 2000). The main idea in the last method is that light correlated with a collective atomic spin via a Faraday interaction can be used as a meter system, reading out one of the spin components. Homodyne measurement of light, as discussed in section II.F then provides information about this spin component, projecting the collective spin into a state with reduced fluctuations in this component.

Means and variances of transverse spin components conditioned on a homodyne measurement of light can be easily evaluated by means of the following classical formulas for mean and variance of a Gaussian random variable $\xi$ conditioned on the measurement of another (possibly correlated) Gaussian random variable $\zeta$ with an outcome $z$,

$$
\left.\langle\xi\rangle\right|_{\zeta=z}=\langle\xi\rangle-\frac{\langle\xi \zeta\rangle}{\left\langle\zeta^{2}\right\rangle} z,\left.\quad \Delta \xi^{2}\right|_{\zeta=z}=\Delta \xi^{2}-\frac{\langle\xi \zeta\rangle^{2}}{\left\langle\zeta^{2}\right\rangle} .
$$

If we assume that both light and atoms are initially prepared in their vacuum state, i.e., atoms are completely polarized along $x$, then the state after the interaction (40) has still Gaussian statistics and the above formulas apply. Assume further that $X_{L}$ is measured on this state with an outcome $x_{L}$. According to the formulas above, the conditional variances of atomic spin components are then given by

$$
\left.\Delta X_{A}^{2}\right|_{X_{L}=x_{L}}=\frac{1+\kappa^{2}}{2},\left.\quad \Delta P_{A}^{2}\right|_{X_{L}=x_{L}}=\frac{1}{1+\kappa^{2}} \frac{1}{2},
$$


and exhibit spin squeezing.

$P_{A}$ is however only squeezed conditioned on the measurement outcome $x_{L}$, since its mean value is given by,

$$
\left.\left\langle P_{A}\right\rangle\right|_{X_{L}=x_{L}}=\frac{\kappa}{1+\kappa^{2}} x_{L}
$$

If we ignore the measurement outcome the evolution is given by Eq. 40 and there is no squeezing since $P_{A}$ is conserved. If a feedback operation is applied to the atoms, the atomic spin can be tilted such that $P_{A}$ is displaced by $-\kappa x_{L} /\left(1+\kappa^{2}\right)$, and the mean value is zero $\left\langle P_{A}\right\rangle=0$. The variances $(68)$ for the (anti)squeezed variances then hold also in the ensemble average.

In Sec. II.F we introduced another method for describing the linear feedback and it is instructive to apply it here. The result $x_{L}$ is fed back on atoms by displacing $P_{A}$ by an amount $g x_{L}$, where $g$ is a suitable gain factor. By Eq. (67), the $P_{A}$ component after feedback is in the ensemble average given by,

$$
P_{A, \text { final }}=P_{A, \text { out }}+g X_{L, \text { out }}=(1+g \kappa) P_{A, \text { in }}+g X_{L, \text { in }} .
$$

Minimizing the variance of $P_{A \text {,final }}$ with respect to the gain $g$, yields an optimal feedback gain $g=-\kappa /(1+\kappa)^{2}$ and the reduced variance of Eq. (68), in agreement with the discussion above. Experiment along these lines has been reported in Kuzmich et al. (2000).

The discussion so far ignores the impairing effects of spontaneous emission and light absorption. In order to take this into account we have to resort to Eq. (56). For the relevant case of small atomic decay, $\eta_{A} \ll 1$, and dominant light losses due to reflection at glass cells and detector inefficiency parametrized by $\epsilon\left(1 \gg \epsilon \gg \eta_{L}\right)$ these equations read

$$
\begin{aligned}
X_{A, \text { out }} & =\sqrt{1-\eta_{A}}\left(X_{A, \text { in }}+\kappa P_{A, \text { in }}\right)+\sqrt{\eta_{A}} f_{X_{A}}, \\
P_{A, \text { out }} & =\sqrt{1-\eta_{A}} P_{A, \text { in }}+\sqrt{\eta_{A}} f_{P_{A}}, \\
X_{L, \text { out }} & =\sqrt{1-\epsilon}\left(X_{L, \text { in }}+\kappa P_{A, \text { in }}\right)+\sqrt{\epsilon} f_{X_{L}}, \\
P_{L, \text { out }} & =\sqrt{1-\epsilon} P_{L, \text { in }}+\sqrt{\epsilon} f_{P_{L}},
\end{aligned}
$$

where we explicitly included Langevin noise operators for atoms $f_{X_{A}\left(P_{A}\right)}$ and light $f_{X_{L}\left(P_{L}\right)}$. For both systems one can to a good approximation assume vacuum properties $\left\langle f_{\alpha} f_{\beta}\right\rangle=\delta_{\alpha, \beta} / 2$. Using these expressions and minimizing the variance with respect to the gain $g$ yields a minimal variance

$$
\Delta P_{A, \text { final }}^{2}=\frac{1+\eta_{A}(1-\epsilon) \kappa^{2}}{1+(1-\epsilon) \kappa^{2}} \frac{1}{2} \geq \frac{\eta_{A}}{2} .
$$

The bound on the achievable squeezing is not so surprising, given that the state of atoms suffered essentially a decay by a fraction $\eta_{A}$. Due to the relation $\kappa^{2}=d \eta_{A}$, cf. Eq. (59), there is always an optimal choice for the decay $\eta_{A}$ given a certain optical depth $d$ (Hammerer et al. 2004). For the decoherence model adopted here the limit to spin squeezing by QND measurement and feedback is $\Delta P_{A \text {,final }}^{2} \geq(1+\sqrt{1+d})^{-1} \sim d^{-1 / 2}$, for large optical density. For a detailed discussion on the limits of spin squeezing by means of Faraday interaction and QND measurement we refer to Bouchoule and Mølmer (2002). The limits strongly depend on the particulars of the QND scheme. For example, for the, so-called, two-color probing (Windpassinger et al. 2008) the $1 / d$ scaling of the squeezing limit is possible.

Our description here covers only feedback where the $i n$ tegrated photocurrent of the homodyne detection is taken as the measurement result and used to correct the atomic state after the probe pulse has passed. It is of course possible to perform a continuous feedback of the photocurrent while the probe pulse is still on. An exhaustive theory for this procedure giving a description in terms of the stochastic Schrödinger equation can be found in Thomsen et al. (2002a b). The paper reporting experimental observation of spin squeezing via continuous QND measurement by Geremia et al. (2004) has been retracted by the authors (Mabuchi, 2008).

It is interesting to note that, beyond atomic interferometry, spin squeezed states received renewed interest in the theory of many particle entanglement. It was shown in Sørensen et al. (2001) that spectroscopic squeezing $\xi_{R}<1$ is a sufficient condition for bipartite entanglement within each pair of spins in the atomic ensemble, see also Wang and Sanders (2003). More general spin squeezing inequalities were fruitfully studied in the context of experimental verification of multipartite entanglement (Korbicz et al. 2005a b; Sørensen and Mølmer, 2001; Tóth et al. 2007).

\section{B. Deterministic entanglement}

The procedure discussed in the previous section can be applied to deterministically create entanglement between two, spatially separated atomic ensembles, as proposed by Duan et al. (2000a). A precursor to this proposal involving entangled light resource has been put forward by Kuzmich and Polzik (2000). Each of the ensembles is described by a collective spin $\vec{J}_{i}(i=1,2)$, or, taking polarizations along $x$ for both systems and adopting the Gaussian approximation, by a pair of canonical operators $\left[x_{A_{i}}, p_{A_{j}}\right]=i \delta_{i, j}$. Consider a probe pulse undergoing Faraday interaction with both ensembles, first with ensemble 1 and then, after propagating some distance, with ensemble 2. By linearity, the state of light is desribed by

$$
\begin{aligned}
X_{L, \text { out }} & =X_{L, \text { in }}+\kappa\left(P_{A_{1}, \text { in }}+P_{A_{2}, \text { in }}\right), \\
P_{L, \text { out }} & =P_{L, \text { in }},
\end{aligned}
$$

c.f. Eq. 40. Note that for both ensembles $P_{A_{i} \text {, out }}=$ $P_{A_{i}, \text { in }}$ is a conserved quantity in the Faraday interaction. Just as for the single ensemble, a measurement of $X_{L, \text { out }}$ will then give a reduced variance of the non-local observable $P_{A_{1}}+P_{A_{2}}$,

$$
\Delta\left(P_{A_{1}}+P_{A_{2}}\right)^{2}=\frac{1}{1+\kappa^{2}}<1,
$$


where the bound corresponds to uncorrelated ensembles in coherent states. Using feedback this non-local squeezing can be achieved unconditionally. In a second step, the spins of both ensembles are rotated by an angle of $\pi / 2$ about the $x$-axis in such a way that

$$
\begin{array}{ll}
P_{A_{1}} \rightarrow X_{A_{1}}, & X_{A_{1}} \rightarrow-P_{A_{1}}, \\
P_{A_{2}} \rightarrow-X_{A_{2}}, & X_{A_{2}} \rightarrow P_{A_{2}} .
\end{array}
$$

A second light pulse interacting with both ensembles as before, will then read out the observable $X_{A_{1} \text {,in }}-X_{A_{2} \text {,in }}$, i.e.

$$
X_{L, \text { out }}=X_{L, \text { in }}+\kappa\left(X_{A_{1}, \text { in }}-X_{A_{2}, \text { in }}\right),
$$

such that a measurement and feedback procedure will produce a squeezed variance of $X_{A_{1}}-X_{A_{2}}$, as before. Note that simultaneous squeezing of these two observables is possible only, because we are are dealing here with commuting observables, $\left[P_{A_{1}}+P_{A_{2}}, X_{A_{1}}-X_{A_{2}}\right]=0$. The counterwise rotation of the two spins about $x$ is therefore crucial. Overall, this will produce a state which fulfills the inequality,

$$
\Delta\left(P_{A_{1}}+P_{A_{2}}\right)^{2}+\Delta\left(X_{A_{1}}-X_{A_{2}}\right)^{2}<2 .
$$

Losses and decoherence will affect this result similarly as the single ensembles spin squeezing in (71). The significance of this inequality is that it constitutes a necessary and sufficient condition for symmetric (with respect to $1 \leftrightarrow 2$ ) Gaussian states of two systems, (Duan et al. 2000b; Simon, 2000).

In the limit of large squeezing, where the variances of both non-local observables vanish, the corresponding state approaches the (unphysical) ideally correlated state with Wigner function $W \sim \delta\left(X_{A_{1}}-X_{A_{2}}\right) \delta\left(P_{A_{1}}+P_{A_{2}}\right)$, which was considered by Einstein, Podolsky and Rosen (EPR) in their famous Gedankenexperiment (Einstein et al., 1935), speculating about the incompleteness of quantum mechanics. See Keyl et al. (2003) for comments on whether and how this limit can be understood in a more rigorous mathematical sense. Because of this connection, the quantity on the right hand side of Eq. 72 is sometimes termed EPR-Variance and denoted by $\Delta_{E P R}$. Its importance is supported by the fact that for symmetric Gaussian states the quantity $r=-\frac{1}{2} \ln \frac{\Delta_{E P R}}{2}$ provides an entanglement measure and uniquely determines the entanglement of formation of the state (Giedke et al. 2003; Wolf et al. , 2004) via $E_{\mathrm{oF}}=\cosh ^{2}(r) \log _{2}\left(\cosh ^{2} r\right)-\sinh ^{2}(r) \log _{2}\left(\sinh ^{2} r\right)$. As follows from the discussion of losses and decoherences, we have to assume a lower limit on the EPR variance $\Delta_{E P R} \gtrsim 2 d^{-1 / 2}$ and thus an upper bound on the bipartite entanglement between the two ensembles of e.g. $E_{\mathrm{oF}} \lesssim 1.15$ ebits $\left(E_{\mathrm{oF}} \lesssim 2.77\right.$ ebits) for an optical density of $d=10(d=100)$. A useful and comprehensive review of the theory of entanglement in systems of continuous variables was recently given by Adesso and Illuminati (2007), for a concise introduction to the basic facts on the same topic see Eisert and Plenio (2003).
Protocol with counter-rotating spins As explained in section Sec. II.E, ensembles at room temperature require a constant magnetic field causing Zeeman splitting, such that scattered light can be detected at sideband frequencies. In the following we will show, that application of an external magnetic field provides in fact an elegant and efficient way to achieve entanglement of two atomic ensembles with a single probe pulse, as was demonstrated in Julsgaard et al. (2001).

In order to show this, we have to resort to the MaxwellBloch equations 65. It is straightforward to generalize these equations to the case of two atomic ensembles and including Larmor precession of the two spins. We assume that the two collective spins precess in opposite directions, which can be achieved taking oppositely oriented fields or parallel fields with atoms prepared in opposite Zeeman substates. Replacing $\omega_{L} \rightarrow-\omega_{L}$ for the second ensemble when using Eqs. 65, the equations of motion for light quadratures are,

$$
\begin{aligned}
\frac{\partial}{\partial z} x_{L}(z, t) & =-\frac{g^{*}(z) \Omega(t)}{\sqrt{2} \Delta}\left[\cos \left(\omega_{L} t\right)\left(p_{A_{1}}(z, t)+p_{A_{1}}(z, t)\right)\right. \\
& \left.+\sin \left(\omega_{L} t\right)\left(x_{A_{1}}(z, t)-x_{A_{2}}(z, t)\right)\right], \\
\frac{\partial}{\partial z} p_{L}(z, t) & =0 .
\end{aligned}
$$

Obviously light reads out sums of momenta and differences of positions, which are commuting observables and is the case for oppositely rotating spins only. It will be instructive to study directly the evolution of these global observables. One finds,

$$
\begin{aligned}
\frac{\partial}{\partial t}\left(x_{A_{1}}(z, t)+x_{A_{2}}(z, t)\right) & =-\frac{g^{*}(z) \Omega(t)}{\sqrt{2} \Delta} \cos \left(\omega_{L} t\right) p_{L}(z, t), \\
\frac{\partial}{\partial t}\left(p_{A_{1}}(z, t)+p_{A_{2}}(z, t)\right) & =0, \\
\frac{\partial}{\partial t}\left(x_{A_{1}}(z, t)-x_{A_{2}}(z, t)\right) & =0, \\
\frac{\partial}{\partial t}\left(p_{A_{1}}(z, t)-p_{A_{2}}(z, t)\right) & =-\frac{g^{*}(z) \Omega(t)}{\sqrt{2} \Delta} \sin \left(\omega_{L} t\right) p_{L}(z, t) .
\end{aligned}
$$

The solutions fall into two groups,

$$
\begin{aligned}
X_{L_{c}, \text { out }} & =X_{L_{c}, \text { in }}+\kappa P_{A_{+}, \text {in }}, & X_{L_{s}, \text { out }} & =X_{L_{s}, \text { in }}-\kappa X_{A_{-}, \text {in }}, \\
P_{L_{c}, \text { out }} & =P_{L_{c}, \text { in }}, & P_{L_{s}, \text { out }} & =P_{L_{s}, \text { in }}, \\
X_{A_{+}, \text {out }} & =X_{A_{+}, \text {in }}+\kappa P_{L_{c}, \text { in }}, & X_{A_{-}, \text {out }} & =X_{A_{-}, \text {in }}, \\
P_{A_{+}, \text {out }} & =P_{A_{+}, \text {in }}, & P_{A_{-}, \text {out }} & =P_{A_{-}, \text {in }}+\kappa P_{L_{s}, \text { in }} .
\end{aligned}
$$

which involve non-local atomic variables

$$
\begin{aligned}
X_{A_{ \pm}} & =\frac{1}{\sqrt{2 L}} \int d z\left(x_{A_{1}}(z) \pm x_{A_{2}}(z)\right), \\
P_{A_{ \pm}} & =\frac{1}{\sqrt{2 L}} \int d z\left(p_{A_{1}}(z) \pm p_{A_{2}}(z)\right)
\end{aligned}
$$


and cosine and sine modulation modes, $X_{L_{c}}, P_{L_{c}}$ and $X_{L_{s}}, P_{L_{s}}$, which were introduced in section II.F on homodyne measurements of ac signals, 66. From the discussion of squeezing in a single cell it is evident that measurement of the sine and cosine component of the $X_{L}$ quadrature will produce a two mode squeezed state with reduced EPR variance 72 .

Implementation Experimental demonstration of deterministic entanglement of two atomic ensembles has been first reported by Julsgaard et al. (2001), with further developments reported by Polzik et al. (2003); Sherson et al. (2006b). The experiments have been performed with Cesium atoms in a vapor at temperatures in the range $15^{\circ}$ $-50^{\circ} \mathrm{C}$. Atoms were contained in glass cells coated from inside with a transparent layer of paraffin (Alexandrov, 2003), as discussed in Sec. III.A. The temperature stabilized cells are placed inside cylindrical magnetic shields, see Fig. 9. Windows made in shields allow for optical axis in two directions - one along the axis of the shield used for optical pumping and another in the radial direction used for the probe light. A solenoid produces a homogeneous axial magnetic field inside each shield. Typical cells have the near-cubic shape with the size of $25-30 \mathrm{~mm}$. The magnetic field inhomogeneity is of the order of $10^{-3}$. This rather modest homogeneity is sufficient since the duration of the light-atoms interaction of $1 \mathrm{msec}$ is sufficiently long so that the atomic motion leads to the effective time averaging of the spatially dependent Zeeman shifts. As a result the magnetic field inhomogeneity has only a quadratic effect on decoherence.

The experimental sequence begins with a a few msec pulse of optical pumping along the direction of the axial magnetic field 9. The level scheme and frequencies of light pulses are shown in Figs. 3 and 4 . The two cells are pumped with the same lasers but the polarization of optical pumping is opposite for them. $99 \%$ or more of the atoms in $F=4$ state are pumped into the $m_{F}=4$ magnetic substate in one cell and into $m_{F}=-4$ in the other cell, as verified by the magneto-optical resonance method (Julsgaard et al., 2004b; Sherson et al. 2006b). The total angular momentum $J_{x}$ of $F=4$ state is calibrated by measuring the Faraday rotation angle of a weak linearly polarized light pulse propagating in the direction of the optical pumping. After optical pumping a probe pulse linearly polarized in $x-y$ plane is fired and its polarization rotation, i.e., the value of the operator $S_{y}$, is measured by two detectors via a balanced polarization measurement, see Fig. 5. The detected photocurrent pulse is sent into the lock-in amplifier which detects the $\cos \left(\omega_{L} t\right)$ and $\sin \left(\omega_{L} t\right)$ components, $X_{L_{c}}$ and $X_{L_{s}}$ introduced in Sec. II.F.

The critical condition for the implementation of the deterministic interface based on homodyne mesaurements is quantum noise limited measurement of light and atoms. This is made possible by employing light and atomic detection at high frequency, typically around
$\omega_{L}=320 \mathrm{kHz}$. Homodyne detectors utilize silicon photodiodes with quantum efficiency more than $98-99 \%$ and low noise photo-amplifiers with the response peaked around $\omega_{L}$. The photodetectors have dark noise equal to shot noise of light for the light power as low as $100 \mu \mathrm{W}$. This means that with a few $\mathrm{mW}$ probe pulse, the detection can be almost perfectly shot noise limited.

The duration of the interaction is chosen to fulfill the condition of optimal entanglement $\kappa_{o p t}^{2}=\sqrt{d}$ with $\eta_{\text {opt }}=1 / \sqrt{d}$. In the experiment the detuning has been chosen within the range $800-1000 \mathrm{MHz}$ to be larger than the Doppler width and the hyperfine splitting of the excited state. Together with the optimal value of the optical power set by the detectors around a few $\mathrm{mW}$, the above conditions lead to the minimal pulse duration of the order of a msec and the corresponding bandwidth in the $\mathrm{kHz}$ range. This value can be in principle increased by reducing the transverse size of the sample and/or using different detectors.

The first experimental run in the presence of atoms is the establishment of the projection noise level of the atomic ensembles. As seen from Eq. (75) the sum variables $X_{A_{-}}, P_{A_{+}}$can be measured in a QND way using a single probe pulse. In the experiment the sequence of the optical pumping followed by the QND measurement is repeated several thousand times and the variance of the measured photocurrent pulses is calculated. The variance is then plotted as a function of the macroscopic spin of the sample, see Fig. 9(c). The linear dependence along with the almost perfect spin polarization proves that the spin noise is at the projection noise level. The projection noise level has been also independently calculated from the macroscopic collective angular momentum of the sample measured via Faraday rotation of the auxiliary probe pulse (Sherson et al. 2006b). The calculated value agreed with the measured projection noise to within $10 \%$ which is well within the uncertainty of this calculation. The projection noise level defines the right hand side in Eq. 72). When normalized to the shot noise of the probe the projection noise value is equal to the total $\kappa^{2}$ of the two samples according to 75 .

After the projection noise level is established the experiment proceeds with generation and verification of the entangled state. In the original paper (Julsgaard et al. 2001) no feedback was applied to atoms and hence a conditional entangled state was demonstrated, that is a non-local state with reduced variance but with a nondeterministic mean value. For possible applications, e.g., teleportation this entanglement is as good as the unconditional one because the knowledge gained with the measurement on the first entangling pulse can be applied to achieve teleportation. The creation of a deterministically and unconditional entangled state has been achieved subsequently (Polzik et al., 2003). The experimental sequence which realizes such entanglement (Sherson et al. 2006b) involves a feedback RF pulse applied to the atoms in between the two probes (Fig. 9p). The feedback pulse of $320 \mathrm{kHz}$ magnetic field with the cosine and sine com- 

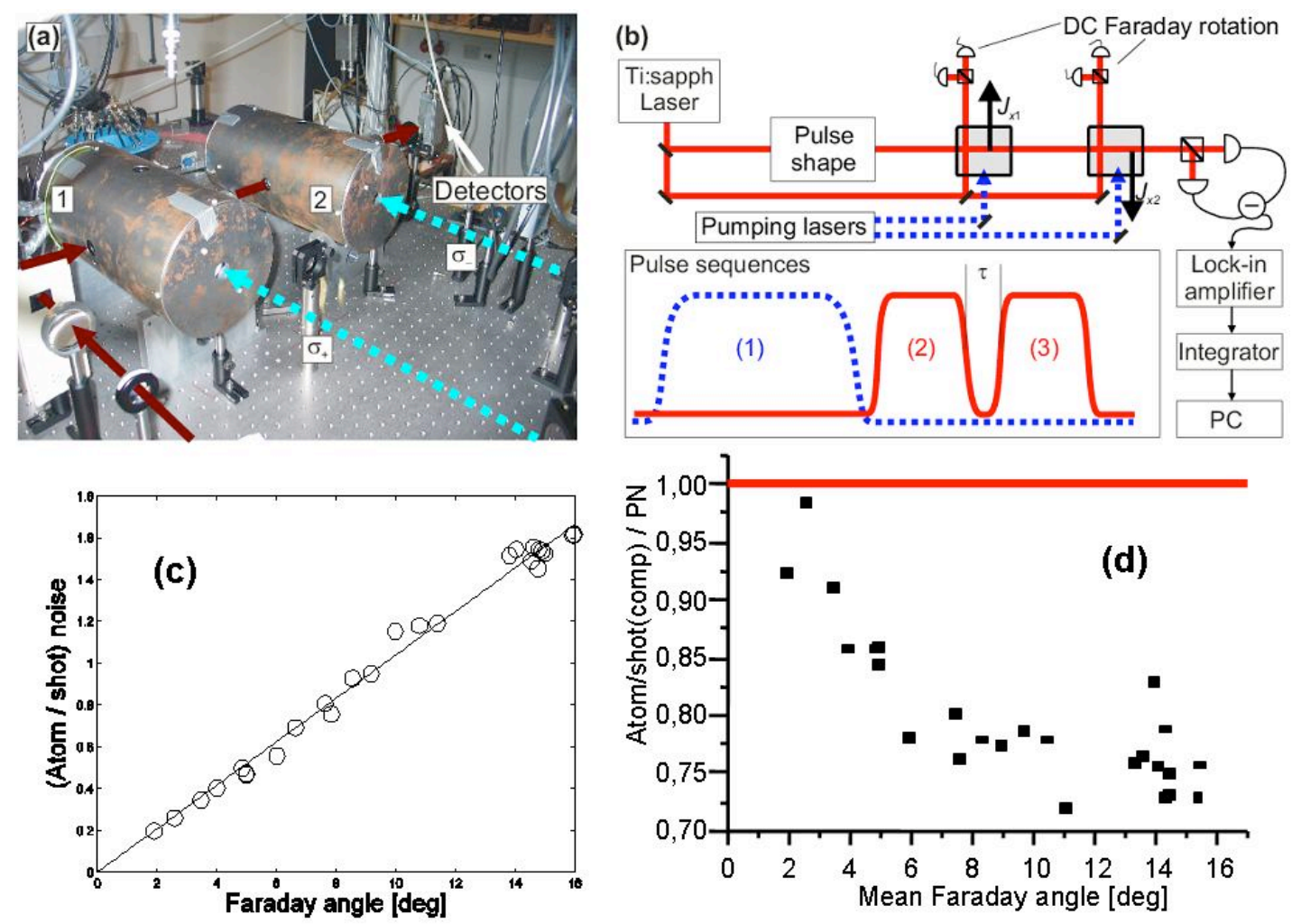

FIG. 9 Deterministic entanglement of two atomic ensembles via QND measurement. a) Experimental setup. Dashed lines - optical pumping, solid arrow - entangling light direction, b) Pulse sequence and the layout of the experiment. (1)-optical pumping pulse, (2)-entangling pulse, (3)-verifying pulse, c) Projection noise of atoms d) EPR variance of the entangled state normalized to the projection noise level

ponents proportional to $X_{L_{c} \text {,out }}$ and $X_{L_{c} \text {,out }}$ respectively is applied to the rf magnetic coils surrounding the cells. An appropriate electronic gain must be chosen so that the feedback pulse rotates the atomic collective spins such as to generate the minimal EPR variance that is the minimal variance of the angle between the spins. The choice of the gain $g=-\kappa /(1+\kappa)^{2}$ minimizes the EPR variance in the absence of decoherence. In the experiment the optimal gain has been chosen operationally by minimizing the EPR variance (see Sherson et al. (2006b) for details). The results for the EPR entangled state of two atomic ensembles are shown in Fig. 9. The variance of the entangled state obtained after applying the feedback pulse is measured by the verifying pulse. Fig. 9 shows this variance normalized to the projection noise variance as a function of the number of atoms. A higher number of atoms leads to a higher value of $\kappa$ and hence to a higher degree of entanglement. The mimimal EPR variance observed in these experiments was $\Delta_{E P R}=1.3$. This variance corresponds to the entanglement of formation of $E_{\mathrm{oF}}=0.28$ ebits.

\section{Probabilistic entanglement}

One of the main motivations for studies of atom light quantum interfaces is an application for quantum repeaters, which would enable long distance quantum communication (Briegel et al. , 1998). A protocol (also known as the DLCZ-protocol) for such a repeater based on atomic ensembles and linear optics was first presented by Duan et al. (2001). Several improvements of the protocol have been suggested (Chen et al. 2007; Jiang et al., 2007: Sangouard et al. 2008; Simon et al. 2007a). As the present review is focused on the deterministic quantum interface, we leave the detailed discussion of probabilistic entanglement and the DLCZ-protocol to a dedicated review and only briefly discuss it here.

The entanglement generation in the DLCZ-protocol uses the parametric gain interaction Eq. (112) between the light and the atoms shown in Fig. 10 a). In principle this interaction could be used in the strong coupling regime $(\kappa \sim 1$ in the notation below) to generate continuous variable entanglement along the lines of the entanglement generation protocol used for quantum teleportation in Sec. VI. Instead the DLCZ-protocol works in the weak coupling limit $(\kappa \ll 1)$ and generates probabilistic entanglement. The first term in Eq. 112 for $a_{A}$ 
a)

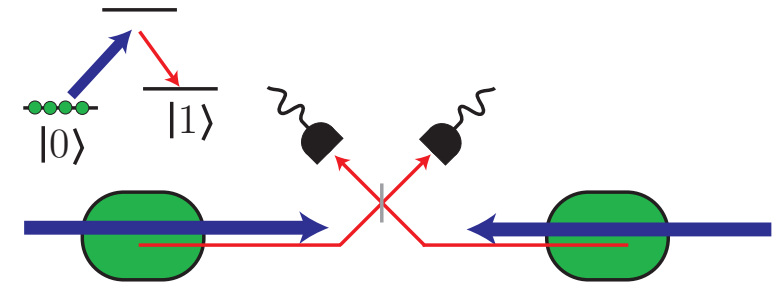

b)

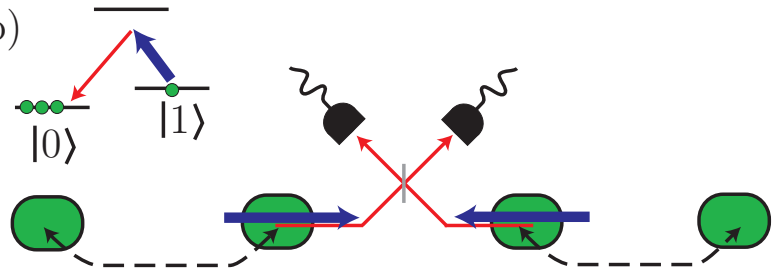

FIG. 10 DLCZ-protocol. a) Entanglement between light and atoms is generated by a parametric gain interaction in two distant ensembles. Photo detection after a beam splitter probabilistically generates entanglement between the two ensembles. b) The excitation in one half of an entangled pair (dashed line) is read out with the beam splitter interaction and mixed on a beam splitter. Photo detection swaps the entanglement so that the outmost ensembles are entangled.

is just the AC-stark shift of the ground state, which can be removed by a simple rescaling, and the phase $\phi$ vanishes for a large detuning. In the limit of weak coupling the dynamics only involves collective operators analogous to the ones defined in Eq. (55) and is equivalent to the evolution with the ideal two mode parametric gain evolution operator $\exp \left(i \kappa\left(a_{L}^{\dagger} a_{A}^{\dagger}+\right.\right.$ H.C. $\left.) / 2\right)$. For $\kappa \ll 1$ the joint state of the collective atomic and light harmonic oscillator degrees of freedom is then

$$
|00\rangle_{A L}+\frac{\kappa}{2}|11\rangle_{A L}+O\left(\kappa^{2}\right),
$$

where $|m n\rangle_{A L}$ describes the state with $m(n)$ excitations in the atomic (light) harmonic oscillator. This result can be understood rather intuitively from the level scheme in Fig. 10(a), which shows that the interaction generates simultaneous excitations of the atoms and the light as described by the expression above.

The state in Eq. (76) is in itself an entangled state, but in the DLCZ protocol it is used to probabilistically generate an entangled stated of two atomic ensembles. The outgoing light modes from two different ensembles are combined on a beamsplitter as shown in Fig. 10. Conditioned on a click in one of the two photodetectors the ensembles are prepared in the state $(|01\rangle \pm|10\rangle) / \sqrt{2}$, because one cannot know from which ensemble the photon was emitted (the sign in the superposition depends on which detector detected the photon). To extend the entanglement to a larger distance the atomic states must be read out from the two atomic ensembles and the outputs mixed on a beam splitter, as shown in Fig. 10(b). Detection of a photon after the beamsplitter extends the entanglement to twice the distance by entanglement swap- ping. This read out process can for instance be done using the beam splitter interaction as discussed in V.D. Note, however, that the atomic mode functions $(29)$ and (32) suitable for the parametric gain and beam splitter interactions are different leading to a mode mismatch if the excitations are read out in the same direction as the entanglement generation (Duan et al. 2002), but this problem may be avoided by reading out in the backward direction (André, 2005).

Following the initial DLCZ-protocol several experiments have been performed, which demonstrate a number of important ingredients of the repeater, of which here we only mention a few. In the first experiments (Chaneliere et al., 2005, Chou et al. 2004: Eisaman et al. 2005: Kuzmich et al., 2003 | Matsukevich and Kuzmich, 2004 van der Wal et al., 2003) it was shown that the number of photons after the read out is correlated with the number of photons emitted during the parametric gain interaction, and that this could be used to generate nonclassical field states. Later experiments (Chaneliere et al., 2007, Chou et al., 2007) have demonstrated entanglement between different atomic ensembles, and recently a probabilistic postselection teleportation experiment was reported (Chen et al. 2008). In the memory chapter below we discuss a recent experiment on storage and retrieval of photonic entanglement (Choi et al. 2008) where atomic entanglement is also present.

\section{QUANTUM MEMORY FOR LIGHT}

Atomic quantum memory for light is an important ingredient for a number of quantum information routines. It is implicit in many quantum communication protocols, in particular in those which require local operations on more than just a single optical pulse, so that storage is necessary. It is required for linear optics quantum computing and for scalable cluster state quantum computing with photons. Quantum memory is a necessary ingredient of a quantum repeater. Different applications demand quantum memories fulfilling different requirements. Some applications require a high-fidelity deterministic write-in operation into the memory. By deterministic we mean a protocol which works with probability one, so that the fidelity is calculated for every try. Others require both write-in and retrieval back to light steps, but can tolerate lower probability of success, provided the success is heralded.

In this chapter we will review several main approaches to the quantum memory for light. We first discuss a figure of merit and a classical benchmark for determining the quality of a quantum memory. We then present the protocol based on a QND interaction and feedback, which was the first to demonstrate a quantum memory channel with the fidelity higher than the classical benchmark. We finally discuss the Raman-based memory, concentrating on the recent achievement of the EIT-based memory experiments, and conclude with the discussion 
of the photon echo memory.

\section{A. Figure of Merit}

From a fundamental perspective quantum memory can be analyzed as a quantum channel, acting in time. A perfect quantum memory is nothing but the identity map taking arbitrary states as input and returning them unchanged, some time later. A realistic memory will be imperfect and the question arises what figure of merit to use in order to evaluate its performance. One sensible measure characterizing the performance of a memory is the average fidelity, i.e. state overlap (Nielsen and Chuang, 2000), which can be achieved between an input state drawn from a predefined set of states according to a predefined probability distribution and the state which is finally read out of the memory. The fidelity is of fundamental relevance if it exceeds the best classical fidelity, in which case the channel is thus outperforming the best classical channel. The classical fidelity relies on the simple strategy of measuring the given quantum state, storing the resulting classical data and on demand reconstructing the quantum state as good as possible.

For example, in the special case where both input and output state are Gaussian with amplitudes $\left\langle X_{\text {in(out) }}\right\rangle,\left\langle P_{\text {in(out) }}\right\rangle$ and variances $\Delta X_{\text {in(out) }}^{2}, \Delta P_{\text {in(out) }}^{2}$ the fidelity is given by

$$
\begin{aligned}
F & =\left[\left(\Delta X_{\text {in }}^{2}+\Delta X_{\text {out }}^{2}\right)\left(\Delta P_{\text {in }}^{2}+\Delta P_{\text {out }}^{2}\right)\right]^{-1 / 2} \\
& \times \exp \left[-\frac{\left(\left\langle X_{\text {in }}\right\rangle-\left\langle X_{\text {out }}\right\rangle\right)^{2}}{2\left(\Delta X_{\text {in }}^{2}+\Delta X_{\text {out }}^{2}\right)}-\frac{\left(\left\langle P_{\text {in }}\right\rangle-\left\langle P_{\text {out }}\right\rangle\right)^{2}}{2\left(\Delta P_{\text {in }}^{2}+\Delta P_{\text {out }}^{2}\right)}\right] .
\end{aligned}
$$

In experiments the average of this fidelity can be taken with respect to a Gaussian distribution of coherent states centered at vacuum with a mean occupation number, $\bar{n}$. If the average fidelity for a flat (infinitely broad) distribution of coherent input states equals unity, the memory is ideal and would store also any Non-Gaussian state perfectly. This follows from the fact that coherent states provide an (overcomplete) basis for the Hilbert space.

It is worth emphasizing that the performance of a quantum memory can be, in principle, tested with coherent states only. Knowing the performance of the memory (a quantum channel) for all coherent states that is performing a quantum tomography of the memory process with coherent states, one can predict the fidelity of the memory channel for an arbitrary class belonging to a single mode. In this sense the often used division between continuous variable memory and discrete variable memory is not quite justifiable. It is more appropriate to speak about the protocols which are based on continuous variable measurements (homodyning) and discrete variable measurements (photon counting).

The question then remains what exactly a measured average fidelity smaller than one guarantees. The benchmark maximum fidelity of a classical channel $F_{\text {class }}$ is known for a limited number of quantum states including qubit states and coherent states with a Gaussian distribution in phase space of width $\bar{n}$. For the latter case Braunstein et al. (2000) conjectured and Hammerer et al. (2005b) proved that

$$
F_{\text {class }}=\frac{1+\bar{n}}{1+2 \bar{n}} \rightarrow \frac{1}{2}, \quad \bar{n} \rightarrow \infty
$$

In the former case, for a class of arbitrary qubit states the maximum classical fidelity is $F_{\text {class }}=2 / 3$ (Massar and Popescu, 1995). Quantum memory which exceeds these classical benchmark fidelities thus shows performance which is classically impossible.

The fidelity is not necessarily the one and only figure of merit for a quantum memory protocol. A relatively high fidelity may still be compatible with errors which are hard to correct. On the other hand, a lower fidelity protocol with particular kinds of errors may be more appropriate for a specific application. For instance the analysis in Brask and Sørensen (2008) shows that different types of errors can have a very different effect on the repeater protocol of Duan et al. (2001), and Surmacz et al. (2006) argue that in certain applications it might be more important to preserve entanglement, when one partner of an entangled pair is stored, than to conserve the quantum state itself.

It is possible to define other figures of merit than the fidelity and also to consider different benchmarks than the one given by a classical measure and prepare strategy, in order to quantify the quality of storage - in quantum memories - but also of transmission of quantum states - as in quantum teleportation, cf. Sec. VI. In the context of teleportation of coherent states, the question of the "right" figure of merit was subject to considerable debate in the literature, see Bowen et al. (2003b); Braunstein et al. (2001); Grosshans and Grangier (2001); Ralph and Lam (1998) and references therein. In particular, Grosshans and Grangier (2001) emphasize the importance of a benchmark $F_{1 \rightarrow 2}$ given by the maximal fidelity achievable in a 1 to 2 cloning machine. For $F>F_{1 \rightarrow 2}$ the memory output is guaranteed to be the best possible copy of the input state. For coherent input $F_{1 \rightarrow 2} \simeq 0.68$, as shown by (Cerf et al., 2005), and is thus more demanding than the classical fidelity benchmark $F_{\text {class }}$, which can also be interpreted as the maximal fidelity of a 1 to $\infty$ cloning machine (Hammerer et al., 2005b). Figures of merit different from fidelity were used in Bowen et al. (2003a b); Hétet et al. (2008b); Ralph and Lam (1998) to characterize both quantum storage and teleportation. There is, however, a consensus that the most sensible figure of merit ultimately depends on the specific application of the quantum memory or teleportation link within a quantum network for e.g. quantum cryptography or optical quantum computation. 


\section{B. QND \& Feedback Protocol}

The first demonstration of a quantum memory (Julsgaard et al., $2004 \mathrm{a}$ ) beating a classical benchmark (Hammerer et al., 2005b) was based on the QND-Faraday interaction of a pulse of light, carrying the quantum state to be stored, with two collective spins counter-rotating in an external magnetic field. The basic input-output equations describing the interaction are given by 75 . Each of these constitutes a realization of the simpler input-output relations 40 for a pulse interacting with a single atomic ensemble without the magnetic field. For simplicity, we will base the theoretical discussion of the main idea on this single ensemble setup, and will return to the actual implementation based on the setup involving two atomic ensembles in the experimental part.

The input light is described by canonical operators $X_{L \text {,in }}$ and $P_{L, \text { in }}$ while the collective spin is prepared in the fully polarized state $\left\langle X_{A, \text { in }}\right\rangle=\left\langle P_{A, \text { in }}\right\rangle=0$ and $\Delta X_{A, \text { in }}^{2}=\Delta P_{A, \text { in }}^{2}=1 / 2$. With a choice of $\kappa=1$ in (40) the entangled state of atoms and light after the interaction is described by

$$
\begin{aligned}
& X_{L, \text { out }}=X_{L, \text { in }}+P_{A, \text { in }}, \quad P_{L, \text { out }}=P_{L, \text { in }}, \\
& X_{A, \text { out }}=X_{A, \text { in }}+P_{L, \text { in }}, \quad P_{A, \text { out }}=P_{A, \text { in }} .
\end{aligned}
$$

Following the interaction the light quadrature $X_{L \text {,in }}$ is measured and the corresponding measurement result $\xi$ is fed back displacing the atomic state such that $P_{A, \text { out }} \rightarrow$ $P_{A \text {,out }}-\xi$. As shown in section II.F the final transformation of the collective atomic spin in the ensemble average is given by

$$
\begin{aligned}
X_{A, \text { out }} & =X_{A, \text { in }}+P_{L, \text { in }}, \\
P_{A, \text { out }} & =P_{A, \text { in }}-X_{L, \text { out }}=-X_{L, \text { in }} .
\end{aligned}
$$

This concludes the mapping of the quantum state of light onto atoms: the mean values are transmitted faithfully (apart from an unimportant phase change) as $\left\langle X_{A \text {,out }}\right\rangle=$ $\left\langle P_{L, \text { in }}\right\rangle$ and $\left\langle P_{A, \text { out }}\right\rangle=-\left\langle X_{L, \text { in }}\right\rangle$. The operator $X_{L \text {,in is }}$ mapped perfectly onto the atomic collective spin, while the operator $P_{L \text {,in }}$ is mapped with the addition of one unit of vacuum operator which comes from the initial coherent state of the atomic ensemble. This latter imperfection can be remedied if the initial atomic spin state is squeezed before the memory operation, such that $\Delta X_{A \text {,in }}^{2} \rightarrow 0$. If such squeezing operation is performed by, for example, an additional QND measurement, the fidelity of the quantum memory operation can, in principle, approach $100 \%$.

In the experiment the quantum memory performance has been tested with a set of coherent states of light taken from a Gaussian distribution of coherent states centered at vacuum with a mean photon number, $\bar{n}$. Given the measured gains and the measured variances, $\Delta X_{A \text {,out }}$ and $\Delta P_{A \text {,out }}$ of the state of the memory, the fidelity can be calculated as:

$$
\begin{aligned}
F=\left(\bar{n}(1-\kappa)^{2}+\right. & \left.1 / 2+\Delta X_{A, \text { out }}^{2}\right)^{-1 / 2} \\
& \times\left(\bar{n}(1-g)^{2}+1 / 2+\Delta P_{A, \text { out }}^{2}\right)^{-1 / 2}
\end{aligned}
$$

As discussed above, if the protocol starts with the atomic ensemble in a coherent state $\Delta X_{A \text {, out }}^{2}=1, \Delta P_{A \text {,out }}^{2}=$ $1 / 2$, and hence $F=\sqrt{3 / 2} \approx 82 \%$ with the choice of $\kappa=$ $g=1$ which is optimal for the class of arbitrary coherent states. For an unknown qubit state $(\alpha|0\rangle+\beta|1\rangle / \sqrt{2}$ the same protocol yields the fidelity of $80 \%$ for optimal values of $g$ and $\kappa$.

The experimental setup and the sequence of operation for writing into the quantum memory is similar to the sequence described in the chapter on deterministic entanglement. The quantum light mode which corresponds to the $\omega_{L}$ sidebands in the polarization orthogonal to the strong field now carries the quantum state of light to be mapped. The state is generated by an electrooptical modulator (EOM) as shown in Fig. 11. The quantum sidebands together with the strong pulse propagate through the atomic cells and are analyzed with the polarization homodyning technique (Fig. 5). The strong pulse thus serves a dual purpose as a strong driving pulse for the interaction with atoms and as a local oscillator for the homodyne measurement.

In the experiment two cells in the magnetic field play the role of one quantum memory unit. As discussed above this approach allows to achieve quantum limited noise for ensembles of trillions of atoms because quantum information is encoded and processed at $\omega_{L}=320 \mathrm{kHz}$ sidebands where classical noise can be strongly suppressed. For two cells with magnetic field according to the equation 75 the light variable $X_{L_{c}}=\sqrt{\frac{2}{T}} \int d t \cos \left(\omega_{L} t\right) x_{L}(t)$ should be measured and the result fed back to the atomic variable $P_{A_{+}, \text {out }}$. The method for measuring $X_{L_{c}}$ by the homodyne measurement of the Stokes parameter $S_{z}$ of the light and the subsequent processing of the photocurrent by the lockin amplifier indicated in Fig. 11 is discussed in section II.F. (66). Note that at the same time the variable $X_{L_{s}}=\sqrt{\frac{2}{T} \int} d t \sin \left(\omega_{L} t\right) x_{L}(t)$ can be measured and fed back into the $X_{A_{-} \text {,out }}$ variable of atoms. The memory could hence be used as a two mode memory, although this direction has not been pursued.

After the projection noise level of atoms has been established, as described in the section on entanglement, the optimal feedback gain must be determined. The gain is chosen to optimize the fidelity for the class of states to be stored in the memory. An example corresponding to the class of coherent states distributed around vacuum with $\bar{n}=8$ is shown in Fig. 12. The optimal values for this class of states are $\kappa=0.8, g=0.8$. After the memory sequence, cf. Fig. 11, is over the atomic variable $P_{A_{+}, \text {out }}$ is measured with a strong QND verifying pulse. The quantum sideband modes of this pulse are initially in the vacuum state. After propagating through the memory 


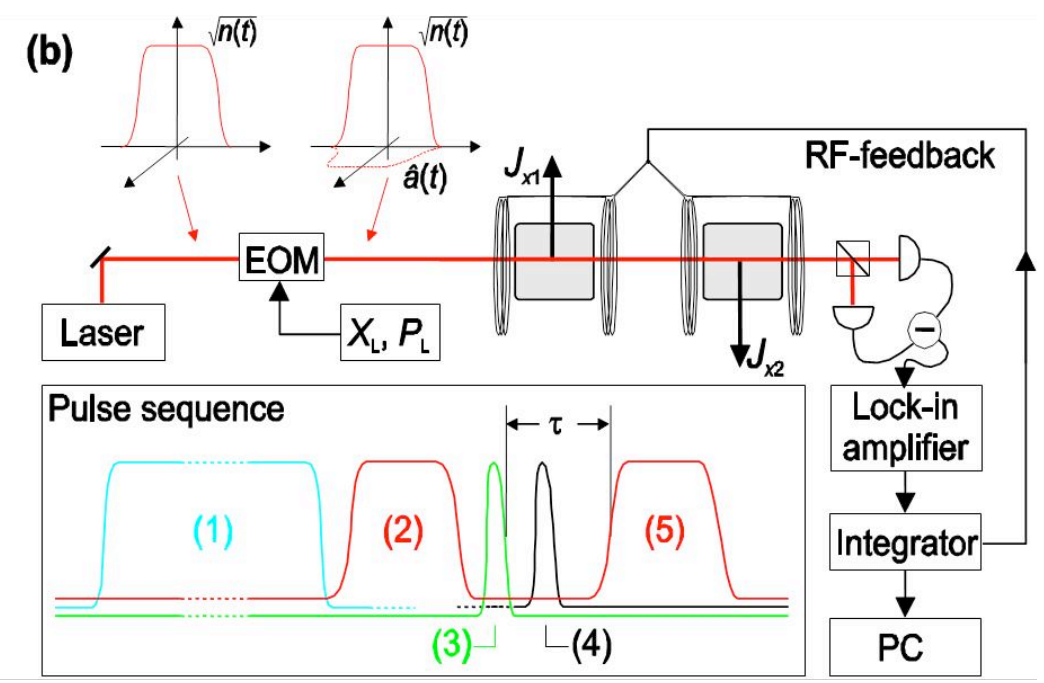

FIG. 11 QND+feedback memory experimental setup and pulse sequence (Julsgaard et al., 2004a). The state of light is encoded by the EOM in the sidebands of the strong pulse $\sqrt{n(t)}$. Two cells serve as the quantum memory unit. The feedback pulse proportional to the photodetector signal is applied to the RF magnetic coils. Inset. (1) - optical pumping, (2) - input pulse, (3) - feedback RF pulse, (4) - RF pulse rotating atomic P into X used for half of verification pulses, (5) - verifying pulse

the quantum sideband modes contain the atomic memory variable $P_{A_{+}, \text {out }}$ according to 75 . Fig. 12 shows that the mean values of this variable for various light input states are very satisfactory proportional to the mean values of the input light canonical variables. As also shown in the figure the same is true for the other canonical variable of light $P_{L_{c}}$ stored in the memory variable $X_{A_{+} \text {,out }}$. To measure $X_{A_{+} \text {,out }}$ in another experimental sequence an $\mathrm{RF}$ pulse rotating the collective atomic spins by $\pi / 2$ and thus converting $P_{A_{+}, \text {out }}$ into $X_{A_{+} \text {,out }}$ has been applied just before the verifying pulse (Fig. 11). The memory is thus shown to work very well as a classical memory for light since the mean amplitude and phase of the input light pulse and the retrieved light pulse are equal to within a chosen factor. Note that as a classical coherent memory this Faraday+feedback memory can have unity retrieval efficiency because the gain is adjustable, whereas for the Raman, EIT, and photon echo-based memories discussed below the efficiency is less than unity in the presence of losses.

The decisive demonstration of the quantum character of the memory follows from the analysis of the variances of the stored quantum state. For the chosen class of the states the benchmark classical fidelity is 52\%. Fig. 13 shows the experimental variances of the atomic memory state for different input light states. From these values the experimental fidelity of the quantum memory can be calculated. Higher than classical values of the fidelity of $64 \%$ have been demonstrated.

\section{Multipass Approaches}

The Faraday+feedback quantum memory, but also the protocols for entanglement and spin squeezing in Sec. IV]

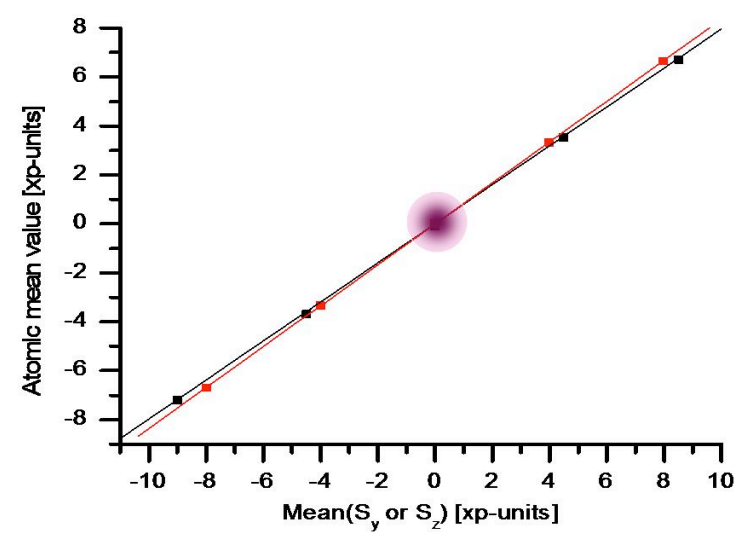

FIG. 12 Atomic coherent memory results (Julsgaard et al. 2004a). The mean values for both quadratures of the input light, atomic memory and the output light are identical to within a chosen factor (here 0.8).

all rely on a pulse of light (or two pulses in the case of Duan et al. (2000a)) interacting with one (or two) cells once. It is of course possible to have one pulse of light interacting several times with one (or more) atomic ensembles, as suggested in several theoretical studies. This section is intended to provide an overview over proposals relying on multipe passes of light through atoms.

Common to all these proposals is that they take advantage of the possibility to perform phase shifts on light and to rotate atomic spins in between the passes. A relative phase shift $\phi$ between the classical field and the quantum field, in $x$ - and $y$-polarization respectively, will 


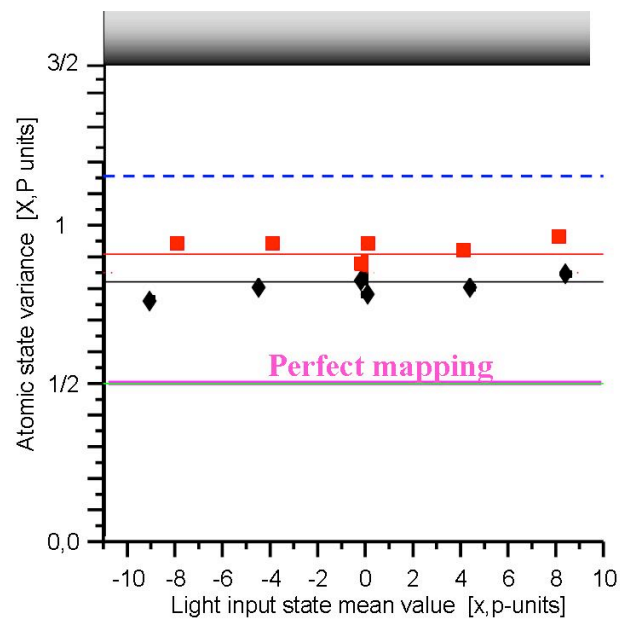

FIG. 13 Variance of the atomic memory state for input coherent states with $n \leq 8$. The variance of $1 / 2$ corresponds to the memory variance equal to the input light variance perfect memory. Above the 3/2 level is the classical memory performance for arbitrary coherent states. The dashed line is the best classical performance for the states within $n \leq 8$ class. Diamonds and squares are experimental results from Julsgaard et al. (2004a).

give rise to a rotation of field qudratures,

$$
X_{L} \rightarrow \cos \phi X_{L}+\sin \phi P_{L}, P_{L} \rightarrow \cos \phi P_{L}-\sin \phi X_{L} .
$$

Rotation of atomic spins about the axis of polarization, via e.g. fast $\mathrm{RF}$ pulses, allows for an analogous rotation of $X_{A}$ and $P_{A}$. Alternatively light can also be directed through atoms from a different direction. As light is sensitive to the projection of the collective atomic spin along the axis of propagation, this is equivalent to a rotation of atoms. Especially for room-temperature atoms in a cell, optical access from two orthogonal directions can be afforded trivially, while light impinging from different sides still talks to the same symmetric mode of atoms due to thermal averaging.

The first proposal along this line is due to Kuzmich and Polzik (2003) and presents a protocol for atomic state read-out, i.e. mapping of the atomic spin state onto the polarization of light. As shown in Fig. 14, in a first pass a pulse of light propagating along $x$ interacts with atoms in a QND fashion, generating a state described by 40 . A phase shift of $\phi=\pi / 2$ then changes $X_{L, \text { in }}^{\prime}=P_{L \text {, out }}$ and $P_{L, \text { in }}^{\prime}=-X_{L \text {,out }}$, where primed variables refer to the second pass of light. The pulse is redirected to the ensemble along the negative $y$ direction, such that the input output relations become, taking $\kappa=1$,

$$
\begin{aligned}
X_{L, \text { out }}^{\prime} & =X_{L, \text { in }}^{\prime}-X_{A, \text { in }}^{\prime}=P_{L, \text { in }}-\left(X_{A, \text { in }}+P_{L, \text { in }}\right)=-X_{A, \text { in }}, \\
P_{L, \text { out }}^{\prime} & =P_{L, \text { in }}^{\prime}=-X_{L, \text { in }}-P_{A, \text { in }} .
\end{aligned}
$$

Aside from an unimportant phase change, the state of atoms is mapped on light. The spurious effect of light noise $X_{L \text {,in }}$ in the second line can be removed by using squeezed light. The overall input-output relations are similar to the ones for quantum memory for light in Eq. 79, but require neither measurement nor feedback.

The previous protocol naturally raises the question whether in principle a perfect state transfer, or state swapping, could be achieved in several passes and specific rotations on atoms and light without using squeezed light. Kraus et al. (2003) addressed this question in full generality and gave neccessary and sufficient conditions for what types of qudratic Hamiltonians can be achieved in two modes, given a specific interaction - such as e.g. the Faraday interaction $\sim P_{L} P_{A}$ - when combined with 'local' operations of the type 80. It was found there, that the Faraday, or QND, interaction is most capable for simulating, in this sense, other interactions, while the beam splitter and parametric gain interaction have no potential to emulate any other interaction. Furthermore, optimal startegies for generation of squeezing and entanglement were devised in the same paper. Fiurăśek (2003) extended these results asking what types of unitary transformations (instead of Hamiltonian interactions) can be achieved and showed in particular that a perfect state swap can be performed with three passages of light, and not for less. Hammerer et al. (2004) applied these general results to the specific situation of a light-matter quantum interface, showing that atomic decay and light losses can be tolerated. See also Kurucz and Fleischhauer (2007) for a discussion of memory conditions and a comparison of these multipass approaches to Raman and EIT based memories.

These protocols all assume a QND interaction in light and matter, which can be achieved for room-temperature atoms only with two cells and counter-rotating spins, as discussed in Sec. IV.B. This makes multiple passes with different directions of propagation a difficult issue. Another complications is due to the fact that roomtemperature ensembles require msec pulses, implying an unreasonable long delay line in the loop of Fig. 14 in order to prevent the pulse meeting itself in the atomic medium. These problems were overcome by Sherson et al. (2006a), Fiurǎśek et al. (2006) and Muschik et al. (2006), who showed that protocols for quantum memory and entanglement generation involving multiple passes of one or more pulses can be matched to Larmor precessing ensembles and that light traversing atoms simultaneously from different directions can in fact be advantageous. In particular, Muschik et al. (2006) consider a single cell Larmor precessing in a magnetic field oriented along $x$ in a setup as shown in Fig. 14, assuming a loop length much smaller than the pulse length, such that the pulse "meets" itself in the medium. For atoms rotating in the sense of the light propagating along the loop, solution of the corresponding Maxwell-Bloch equations, taking care of propagation effects, yields input-output relations for 
atomic variables,

$$
\begin{aligned}
& X_{A, \text { out }}=e^{-\kappa^{2} / 2} X_{A, \text { in }}+\sqrt{1-e^{-\kappa^{2}}} X_{L, \text { in }}^{+}, \\
& P_{A, \text { out }}=e^{-\kappa^{2} / 2} P_{A, \text { in }}+\sqrt{1-e^{-\kappa^{2}}} P_{L, \text { in }}^{+},
\end{aligned}
$$

where $X_{L, \text { in }}^{+}, P_{L \text {,in }}^{+}$refer to a light mode centered at the upper side band frequency $\omega_{0}+\omega_{L}\left(\omega_{0}\right.$ is the carrier frequency of the classical driving pulse and $\omega_{L}$ the Larmor frequency) with a weakly exponentially decaying mode function. $\kappa$ is again given by (41). An analogous inputoutput relation holds for light, such that this scheme realizes an exponentially efficient state exchange of atoms and light.

The Faraday interaction was achieved as a sum of the beam splitter and the parametric gain interaction, c.f., Fig. 2. What we effectively achieve by having multiple passes is that we add two Faraday interactions with different relative phases between the beam splitter and parametric gain interactions in Fig. 2 (c). By suitable geometries and phase shifts the parametric gain interactions from the two passes cancels, and we are left with the beam splitter interaction making an ideal memory transformation. With other configurations it is the beam splitter interaction which cancels and the parametric gain interaction takes effect, generating an entangled state of light and atoms, whose EPR variance, cf. Sec. IV.B, scales asymptotically as $\Delta_{E P R} \sim \exp \left(-\kappa^{2}\right)$ (Muschik et al. 2006).
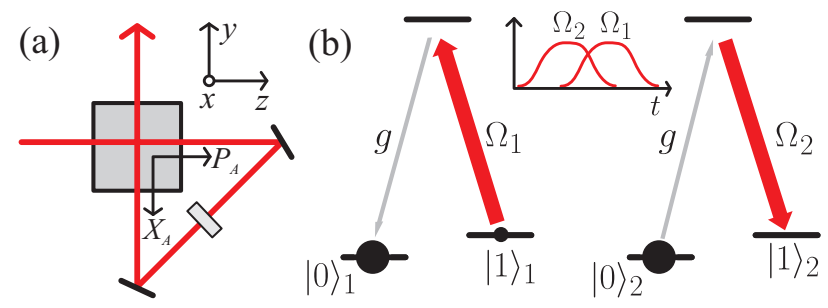

FIG. 14 (a) Setup for two pass protocol for read-out of atomic states as suggested by Kuzmich and Polzik (2003) and, with an additional magnetic field applied along $x$, for full state exchange or creation of entanglement between light and atoms as suggested by Muschik et al. (2006). (b) State swap between two ensembles coupled to a common cavity mode based on adiabatic passage as demonstrated by Simon et al. (2007b). The classical pulses $\Omega_{i}$ are applied in a counterintuitive sequence.

\section{Raman and EIT approach}

The beam splitter interaction is a quite natural choice for a quantum memory since the interaction maps excitations from light to atoms and back. The beam splitter interaction was proposed for a quantum memory by Kozhekin et al. (2000) who considered the far-detuned Raman limit $\Delta \gg d \gamma$. Later this Raman limit was also considered by Nunn et al. (2007). Most of the attention to the beam splitter interaction, however, came with the realization that on resonance with the atomic transition, Electromagnetically Induced Transparency (EIT) can be used for quantum memory for light. The principles of EIT and its applications for coherent memory for light has been reviewed earlier (Fleischhauer et al., 2005; Lukin, 2003), hence we will concentrate here mostly on the recent advances on quantum memory. The EIT is achieved when a strong control optical pulse renders the $\Lambda$ system transparent for the signal pulse, see Fig. 1(b). This transparency is accompanied by a strong reduction in the group velocity of the signal pulse. The result is that the signal pulse entering the atomic ensemble is spatially compressed. If the compression is sufficient to make the signal pulse fit inside the sample, the control field can be turned off at this point and the signal pulse is "frozen" into the atomic ground state coherence - the dark state polariton wave. The process can be inverted by turning on the control field after some delay, which leads to the generation of a signal pulse which "remembers" the classical and quantum properties of the input signal pulse.

To see what happens in this situation let us now consider the $\Delta \rightarrow 0$ limit of the solution in Eqs. 46), (47), and 50 . If we assume a sufficiently large incident classical driving field so that $h(0, t) z / L \gg 1$ we may use the asymptotic form of the Bessel function and write the integral kernel 47) as

$$
m(\Omega ; t, z) \approx \sqrt{v_{g}} \sqrt{\frac{d}{L}} \frac{1}{2 \sqrt{2 \pi} \sqrt[4]{v_{g} t z}} \mathrm{e}^{-d\left(\sqrt{v_{g} t}-\sqrt{z}\right)^{2} / L},
$$

where we have for simplicity assumed that the classical driving field is time independent and have introduced the group velocity

$$
v_{g}=\frac{\Omega^{2} L}{\gamma d}
$$

For a large optical depth this kernel is centered around $z=v_{g} t$. If the spin wave mode is slowly varying on a length scale $L / \sqrt{d}$, or if the incoming field is slowly varying on a time scale $L / \sqrt{d} v_{g}$, i.e., the input field is inside the EIT-transparency window (Fleischhauer et al. 2005), the expression above can be approximated by a delta function $m=\sqrt{v_{g}} \delta\left(z-v_{g} t\right)$. The solutions of Eqs. (46) and (50) then become

$$
\begin{aligned}
a_{A, \text { out }}(z) & =\frac{1}{\sqrt{v_{g}}} a_{L, \text { in }}\left(T-z / v_{g}\right) \\
a_{L, \text { out }}(t) & =\sqrt{v_{g}} a_{A, \text { in }}\left(L-v_{g} t\right) .
\end{aligned}
$$

The first line describes the writing of the input field into the atomic memory, whereas the second one describes the readout of the atomic memory back into light. To accomplish the writing (storage) process the control field must be turned off when the entire input pulse is inside the medium, which requires $T_{\text {signal }}<L / v_{g}$. This prompts the reduction of $v_{g}$ to zero and hence "stopping" of light. The process of the readout or the retrieval is accomplished according to the second line by turning the 
control field on again which leads to the mapping of the atomic memory operator back onto the light operator.

Recently several experimental implementations of the EIT atomic memory (Chaneliere et al., 2005, Choi et al., 2008, Eisaman et al. 2005. Kuzmich et al. 2003) have demonstrated that such quantum features of light as violation of a Cauchy-Schwarz like inequality and entanglement can be preserved by the memory. Very recently the EIT based memory for quantum fluctuations has been also demonstrated using squeezed vacuum light in (Appel et al., 2008, Honda et al., 2008). As mentioned in (Honda et al. . 2008) the fidelity calculation is misleading for the case studied in these two papers since only one particular state has been used and, in addition, the overlap of this weakly squeezed state with vacuum is not far from unity. The overall efficiency of storage and retrieval of around $10-15 \%$ has been achieved, and weakly squeezed light (around $-0.2 \mathrm{~dB}$ ) has been retrieved from the memory.

The experiments (Chaneliere et al. 2005, Choi et al. 2008, Eisaman et al., 2005 Kuzmich et al., 2003) have had a relatively low overall efficiency of the storageretrieval process, however it did not preclude observation of the storage of non-classical light. The reason is that the violation of the Cauchy-Schwarz inequality is based on the measurement of the normally ordered second order correlation function $g^{2}(1,2) \equiv\left\langle: \hat{n}_{1} \hat{n}_{2}:\right\rangle /\left\langle\hat{n}_{1}\right\rangle\left\langle\hat{n}_{2}\right\rangle$ where $\hat{n}_{1,2}$ denote photon number operators measured at spacetime points $1,2 . g^{2}(1,2)$ which describes the normalized probability of detecting a photon at point 2 conditioned on the detection at point 1 is insensitive to losses because they affect the numerator and the denominator in the same proportion. For an ideal single photon state $g^{2}(1,2)=0$, whereas any $g^{2}(1,2)<1$ is a signature of the non-classical character of the field which for the case of a stationary photon flux is referred to as photon antibunching.

The source of the non-classical field used in Chaneliere et al. (2005), Eisaman et al. (2005), and Choi et al. (2008) has been an atomic ensemble prepared in an approximate atomic single excitation state by a weak parametric gain interaction (see Sec. IV.C) which was then retrieved onto light by a beam splitter process. Nonclassical state prepared in this way have been reported by Balić et al. (2005); Chaneliere et al. (2005); Chou et al. (2004); Du et al. (2008); Eisaman et al. (2005); Kuzmich et al. (2003); Matsukevich and Kuzmich (2004); van der Wal et al. (2003). The sequence is similar to that described in Sec. IV.C except for only one atomic ensemble is involved at this stage. The layout of the experimental setup of Eisaman et al. (2005) is shown in Fig. 15. The weak parametric gain interaction driven by the "write" classical field so that $\kappa \ll 1$ creates a single atomic excitation in the "source" ensemble conditioned on the detection of a photon in a particular spatial "Stokes" mode. The requirement of the low gain $\kappa \ll 1$ is necessary so that the multi-photon pulses are suppressed, which is a typical situation for the parametric-type interaction. The "retrieve" strong pulse converts the atomic excitation into "anti-Stokes" light pulse. The non-classical character of this field is manifested by the correlation function conditional on the detection of one Stokes photon $g^{2}\left(A S \| n_{S}=1\right)<1$. This condition means that if a Stokes photon has been detected and if there is a photon in the anti-Stokes pulse, then the probability of having a second photon in the anti-Stokes pulse is less than that for a random process. In the ideal case this probability is zero and the anti-Stokes pulse contains either no photons or just a single one.

The non-classical light pulses produced by the "source" ensemble are then directed towards the memory ensemble. There they are stored and retrieved using the EIT pulse sequence as shown in Fig. 16 after Choi et al. (2008). The figure shows the probability of detecting a photon after the storage medium. The strong "write" driving EIT field has a constant amplitude until the quantum input pulse appears at $\tau=0$. Then the driving field is turned off leading to the storage of the light in the medium. For ideal storage there should be no counts corresponding to the input pulse. The counts around $\tau=0$ hence correspond to the "leakage" of light through the memory ensemble. After a delay time of $1 \mu \mathrm{sec}$ the strong "read" control field is applied and the atomic excitation is read out generating a light pulse as prescribed by Eq. 50. An overall storage-retrieval probability of $17 \%$ has been shown in Choi et al. (2008). This experiment, as well as the experiment by Chaneliere et al. (2005) used $\mathrm{Rb}$ atoms cooled and trapped in a magneto-optical trap (MOT), whereas Eisaman et al. (2005) used Rb atoms in a neon buffer gas cell at room temperature. In the two former experiments a small sub-ensemble of atoms contained within the volume of the focused optical beam inside the sample served as the memory. For this reason the storage time was restricted by the motion of atoms to about a $\mu$ sec. In both experiments the two lower levels of the $\Lambda$ system were the two ground state $5 S_{1 / 2}$ hyperfine levels and the upper level was the $5 P_{3 / 2}$ level.

The results for the conditional correlation function $g^{2}$ (out $\left.\| n_{S}=1\right)<1$ for the memory output field obtained by Chaneliere et al. (2005) are shown in Fig. 17. The results clearly show the nonclassical character of the retrieved light. The overall efficiency of the storage/retrieval process of a photon was $6.4 \%$. Similar results have been obtained by Eisaman et al. (2005).

The experiment by Choi et al. (2008) have taken the EIT approach one step further by demonstrating it for an entangled state of a photon (Fig. 18). In this experiment a conditional non-classical state with $g^{2}(A S \|$ $\left.n_{S}=1\right)<1$, an approximate single photon state, was split on a beam splitter in two parts. Conditioned on the registration of the Stokes photon in the source ensemble (not shown in Fig. 18) the input consisted of a single photon with $15 \%$ probability. The rest was mostly vacuum with a small addition of a two-photon component with the probability of $9 \%$ of that for a Poisson source with the same average photon number. The input state with 


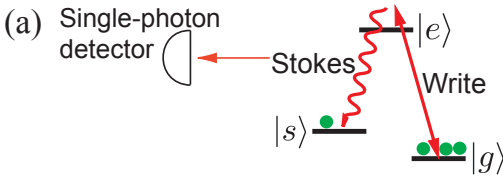

Source atoms

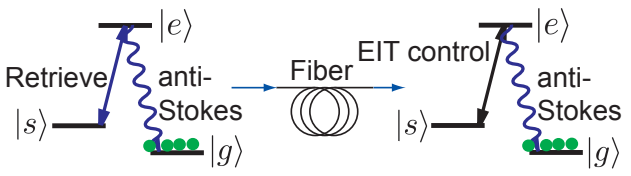

Target atoms

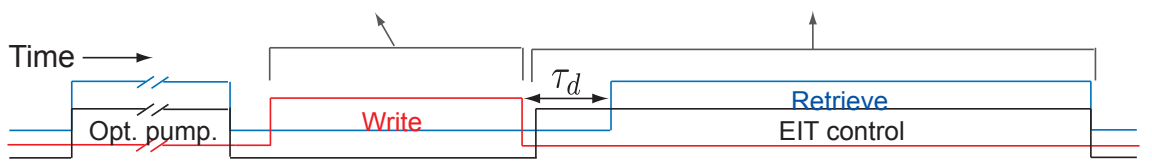

(b)

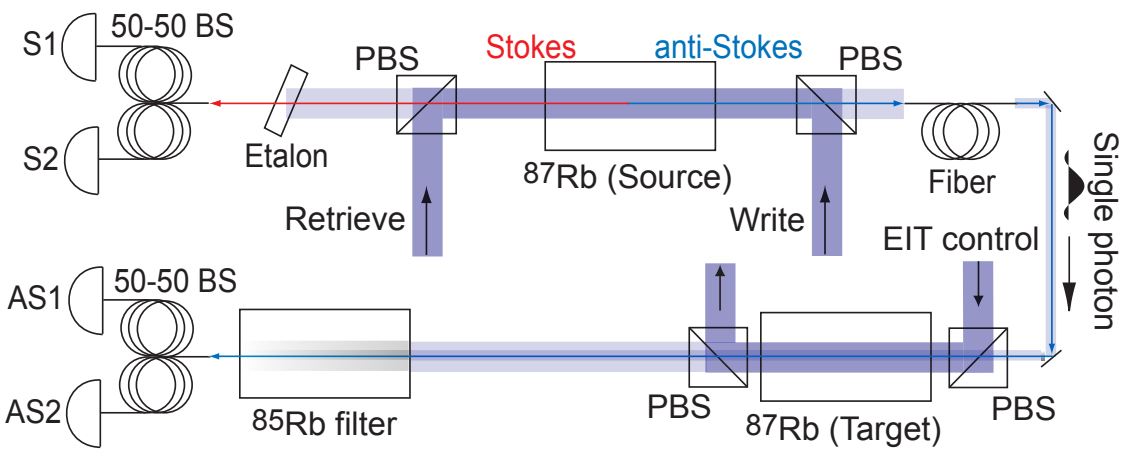

FIG. 15 EIT-based memory setup after Eisaman et al. (2005). a) Level scheme and sequence of control pulses. Left - parametric interaction for a probabilistic generation of an excitation in the source ensemble, center and right - EIT-based beam splitter interactions for storage and retrieval. b) Setup showing the source ensemble of the non-classical photon flux and the memory ensemble (target atoms). A count in detector S1 or S2 is a condition for starting the process. Detectors AS1 and AS2 analyze the statistics of the input and output memory photons.

these properties has the concurrence of 0.10 corresponding to an entanglement of formation of $E_{\mathrm{oF}}=0.025$ ebits. (A concurrence of unity corresponds to $E_{\mathrm{oF}}=1$ ebits, i.e. to a maximally entangled Bell state.) The input state was linearly polarized at $45^{\circ}$ with respect to the polarizing beam splitter (Fig. 18). The photonic state of the two outputs of the beam splitter conditioned on the successful preparation of the single photon state is $\Psi_{\text {in }}=\left(\left|0_{L}\right\rangle\left|1_{R}\right\rangle+e^{i \varphi}\left|1_{L}\right\rangle\left|0_{R}\right\rangle\right) / \sqrt{2}$. The two components of this state have been directed into two atomic ensembles. The two ensembles were two groups of atoms within a Magneto-optical trap (MOT). Rb atoms were optically pumped into a particular magnetic sublevel of the ground state $F=4, m_{F}=0$ which contributed to a better performance of the memory.

After the EIT write and retrieve steps similar to those described earlier in this section the two retrieved components were combined on a polarizing beam splitter. With a suitable choice of the phase the state recreated from an ideal memory would make the single linearly polarized photon, just like the input one. Changing the phase between the two components of the state stored in the two ensembles by adjusting the $\lambda / 2$ wave plate Choi et al. (2008) performed tomography of the entangled state and obtained the density matrix of it. The results of this procedure are shown in Fig. 19. From these results the concurrence of the retrieved state of $0.017\left(E_{\mathrm{oF}}=0.062\right.$ ebits $)$ has been inferred demonstrating that the retrieved state has retained entanglement after the storage process.

The overall efficiency of the storage/retrieval of entanglement measured by the ratio of the concurrence of the output to the concurrence of the input is $20 \%$ after the storage time of $1.1 \mu \mathrm{sec}$. It is limited by the finite optical depth of the sample and can be improved by optimization of the pulse shapes as discussed below.

These experiments represent exciting experimental progress, however a higher efficiency is still desirable for future applications. Higher efficiency can be achieved with an increased optical depth, but also by optimizing the shape of the classical driving field $\Omega(t)$. This optimization problem is the subject of a series of papers by Gorshkov et al. (2007a b c d, 2008) (see also related work by Dantan et al. (2005, 2006b); Dantan and Pinard (2004) and by Nunn et al. (2007)). These studies show that for any slowly varying pulse of duration $T$ such that $T d \gamma \gg 1$ the optimal storage and retrieval efficiency is the same and is independent of the detuning from the excited atomic state. Furthermore, the optimized inefficiency only depends on the optical depth $d$ and scales as $1 / d$. These results can be understood from a new "universal" physical picture of the storage and retrieval 


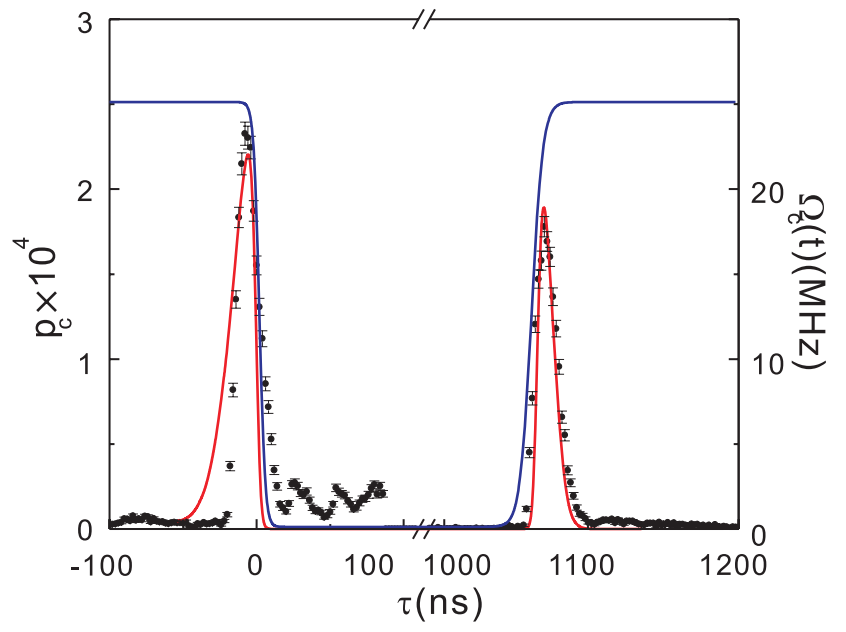

FIG. 16 EIT-based memory (Choi et al. (2008)). Probability of detecting a photon downstream the memory ensemble (left axis). The points around $\tau=0$, the moment when the input pulse is launched are due to the imperfect memory leading to the "leakage" of photons through it. The strong field (right axis) is turned off around $\tau=0$ and turned on again at $\tau=$ $1 \mu$ sec leading to the retrieved pulses with the overall storage retrieval probability of $17 \%$
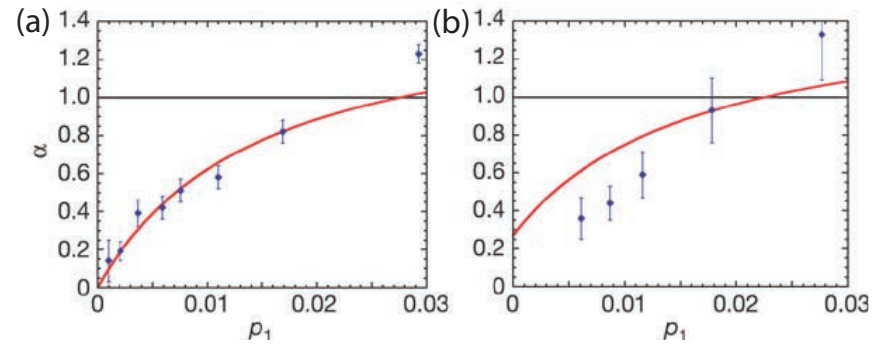

FIG. 17 The correlation function for the input (b) and output (d) light for the EIT-based memory from Chaneliere et al. (2005). $g^{2}(0)<1$ is a signature of non-classical character corresponding to the single photon in the limit of $g^{2}(0)=0$.

process: First of all for a given stored spin wave, the retrieval process is essentially a constructive interference effect similar to super radiance, where the radiated fields from all atoms interfere constructively in a certain direction. As a result there is a fixed branching ratio between the decay into the desired quantum field mode and the decay into all other modes, which is independent of the control field shape as long as sufficient optical power is used. Secondly, the optimal storage is the time reverse of retrieval and, by time reversal symmetry, the optimal storage efficiency is identical to the retrieval efficiency.

The retrieval efficiency does, however, depend on the spin wave mode, and for optimal storage the classical drive field $\Omega(t)$ has to be chosen so that it maps an incoming field mode into the optimal spin wave. This optimal field shape $\Omega(t)$ can be found by a direct calculation, but an alternative experimental procedure for finding optimal shapes is demonstrated by Novikova et al. (2007). In this experiment which worked in the classical regime with many photons in the signal beam, the shape of the pulse to be stored was optimized for a given classical drive field. Novikova et al. (2007) first stored a given pulse and recorded the shape of the retrieved pulse. Then the time reverse of the recorded pulse was used as an input for the next round of storage, retrieval and measurement. This procedure rapidly converges and yields the optimal efficiency (Gorshkov et al. 2007c), which in the experiment was in the range $42-45 \%$.

Because the optimal strategy for storage and retrieval is based on time reversal, higher efficiency can actually be achieved if the excitation is read out in the backward direction compared to the direction of storage (Gorshkov et al. 2007c). This change of direction, however, requires a redefinition of the atomic operators $(29)$. The mode functions $u_{m}\left(z ; \vec{r}_{\perp}\right)$ in Eq. (29) is a solution to the 3dimensional Maxwell equation in the forward direction but may not be so in the backward direction, and this will complicate the dynamics (for a discussion of a related problem see André (2005)). Unless the mode function can be chosen real, which requires a Fresnel number much bigger than unity. Furthermore a finite energy difference $\omega_{01}$ between the two ground state introduces a momentum difference $\Delta k=\omega_{01} / c$ which reduces the storage efficiency unless $\Delta k L \ll 1$ (Gorshkov et al. , 2007c). A way to cope with this problem is presented by Surmacz et al. (2007).

For applications in a quantum network, stored excitations will have to be processed in the quantum memory. A first step in this direction was performed in a recent experiment by Simon et al. (2007b) with two atomic ensembles in a medium Finesse $(\mathcal{F}=240)$ cavity. It demonstrated adiabatic transfer of a single excitation stored in one ensemble to the other ensemble with the cavity mode serving as a quantum bus, as well entanglement of the two ensembles by a partial transfer. First, a single excitation is generated in one ensemble by driving a weak parametric gain interaction with subsequent detection of a Stokes photon, as described above. Conditioned on the successful generation of a single excitation in ensemble 1, beam splitter interactions are switched on and couple both ensembles to a common cavity mode, as indicated in Fig. 14(b). This is done adiabatically, first for the "empty" ensemble 2 and then for ensemble 1, in a counterintuitive sequence generating an adiabatic darkstate passage $|1\rangle_{1}|0\rangle_{2} \rightarrow|0\rangle_{1}|1\rangle_{2}$. After the transfer, the single excitation was read out from ensemble 2 , demonstrating a transfer efficiency between $10 \%$ and $25 \%$, depending on the optical depth. Simon et al. (2007b) also 


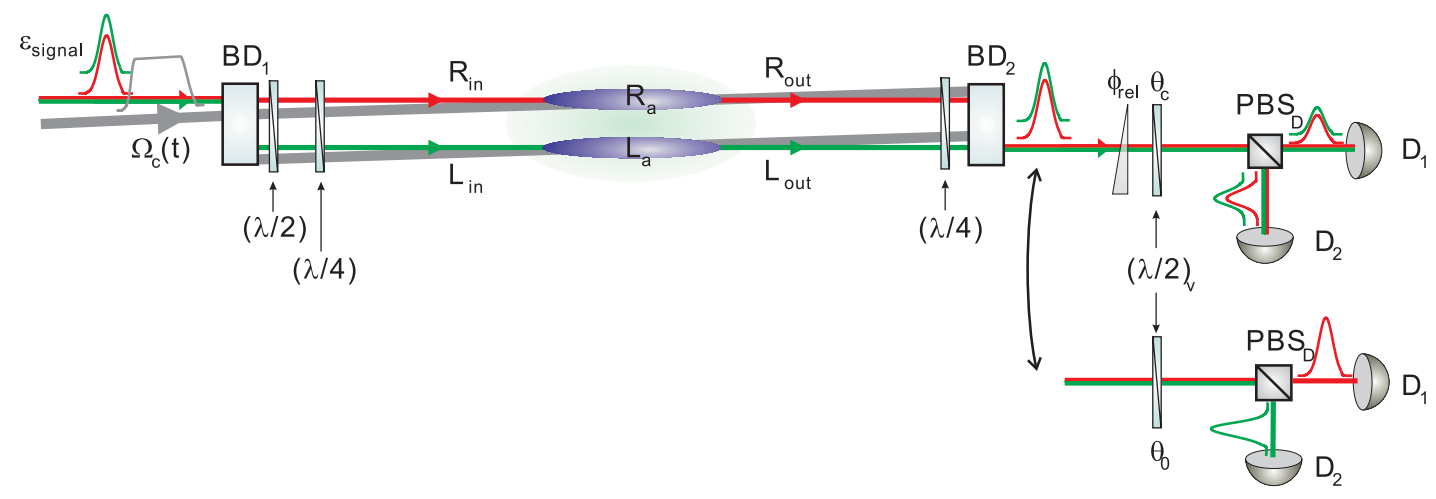

FIG. 18 EIT-based memory for an entangled state (Choi et al., 2008). Two memory ensembles store two components of an entangled state which are later retrieved and analyzed by two pairs of coincidence detectors $D_{1,2}$.

(a) $\rho_{\text {in }}$

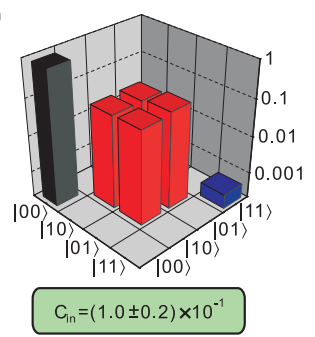

(b) $\rho_{\text {out }}$

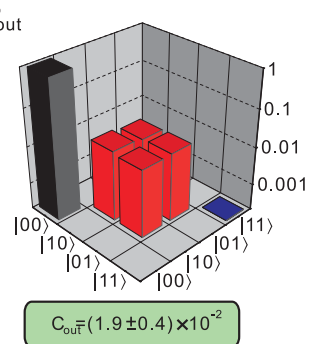

FIG. 19 Characterization of the retrieved entangled state (Choi et al. 2008). The reconstructed density matrices of the input (b) and the output (c) states of the light.

demonstrated a partial swap of the excitation, generating in the ideal case an entangled state of the ensembles, $|1\rangle_{1}|0\rangle_{2} \rightarrow \cos \theta|1\rangle_{1}|0\rangle_{2}+\exp (i \phi) \sin \theta|0\rangle_{1}|1\rangle_{2}$, where $\theta$ is controlled via the intensities and $\phi$ by the relative phase of the laser fields $\Omega_{i}$ in Fig. 14(b). Reading out the collective excitations and measuring photon correlation functions, similar to what was done by Chou et al. (2005), a lower bound on the entanglement of the ensembles was determined, giving a concurrence larger than 0.0046 corresponding to an entanglement of formation of $E_{\text {of }} \geq 0.0001$ ebits (Wootters, 1998).

\section{E. Photon Echo}

It has long been know that the photon echo technique (Kurnit et al. 1964) could be used to store classical light pulses. Recently it has been realized that one can extend these techniques into the quantum interface domain (Kraus et al. 2006 : Moiseev and Kröll, 2001). If a light pulse is absorbed by an ensemble of two-level atoms, the quantum properties of light will be stored in atoms as shown already in the early experiment Hald et al. (1999). The problem is that for a meaningful memory the coherence time of atoms should be longer than the duration of the pulse. However, this means that the bandwidth of the interaction which is set by the inverse coherence time

is narrower than the bandwidth of the light set by its inverse duration, so that the entire pulse cannot be stored. A way out of this problem is to use a medium with inhomogeneous broadening. Then different frequency components of the light pulse will be effectively stored in different sub-groups of atoms. To avoid the dephasing of the stored state caused by the broadening a photon echo technique is used which also allows to control the release of the stored excitation.

The essence of the photon echo approach is to have an inhomogeneously broadened line which is then reversed. In the original approach an incoming light field is absorbed by a two level system with a broadened optical transition. Due to the inhomogeneous broadening the optical coherence from each atom $(i)$ precesses at different frequencies $\exp \left(-i \omega_{i} t\right)$. This different precession frequencies dephase the optical polarization such that it does not radiate because the radiation from different atoms interfere destructively. In the simplest version of the photon echo, a strong $\pi$-pulse, which interchanges the ground and excited states, is applied after a time $T / 2$. This strong $\pi$ pulse effectively reverses the phase acquired by each atom such that the subsequent time evolution causes a rephasing of the optical coherence. At time $T$ all the atomic polarizations are again in phase causing an echo signal to be emitted.

A modification of the photon echo technique called Controlled Reversible Inhomogeneous Broadening (CRIB) which in principle allows an ideal read out efficiency was introduced by Moiseev and Kröll (2001) and further developed by Kraus et al. (2006). In the first work a $\Lambda$-system similar to Fig. 1(b) was used. The photon is absorbed while an applied electric field broadens the $|0\rangle-$ $|e\rangle$ absorption line. A $\pi$ pulse traveling in the opposite (backward) direction is applied, which takes the population form the excited state to the initially empty state $|1\rangle$ for long term storage. Another $\pi$-pulse traveling in the backward direction is applied, which releases the excitation. During retrieval the broadening with the applied electric field is reversed, which reverses the time evolution resulting in an ideal retrieved signal in the backward direction if one ignores the excited state decoherence. 
Experimentally this scheme was realized by Alexander et al. (2006). Staudt et al. (2007) demonstrates that similar photon echo techniques can preserve the coherence of time bin qubits conditioned on having an outgoing photon.

In a recent experiment (Hétet et al., 2008a) CRIB has been implemented in a particularly elegant and simple way, which was also considered by Sangouard et al. (2007). In contrast to most other techniques considered in this paper, no strong classical control pulse is required in this scheme. Hétet et al. (2008a) applied a gradient electric field to the crystal used for the memory. Light interacted with $\mathrm{Pr}^{3+}$ ions which were doped into a $\mathrm{Y}_{2} \mathrm{SiO}_{5}$ host. Due to the gradient field resonant frequencies of the ions at different parts of the crystal were distributed by the Stark effect within the $2 \mathrm{MHz}$ bandwidth. The homogeneous line width of the ions is $100 \mathrm{kHz}$. Hence the ions should have been in principle capable of storing a pulse of light for the time which is 20 times longer than the pulse duration. The stored light has been released by reversing the sign of the electric field gradient. In the experiment a classical pulse of $2 \mu \mathrm{sec}$ duration was stored for $2 \mu \mathrm{sec}$. An overall efficiency of the storage and retrieval of $15 \%$ has been achieved. The results for the echo memory for classical pulses are shown in Fig. 20. The figure shows the transmitted part of the input pulses (centered around zero) and stored and retrieved pulses for different storage times. All pulses are normalized to the amplitude of the input pulse.

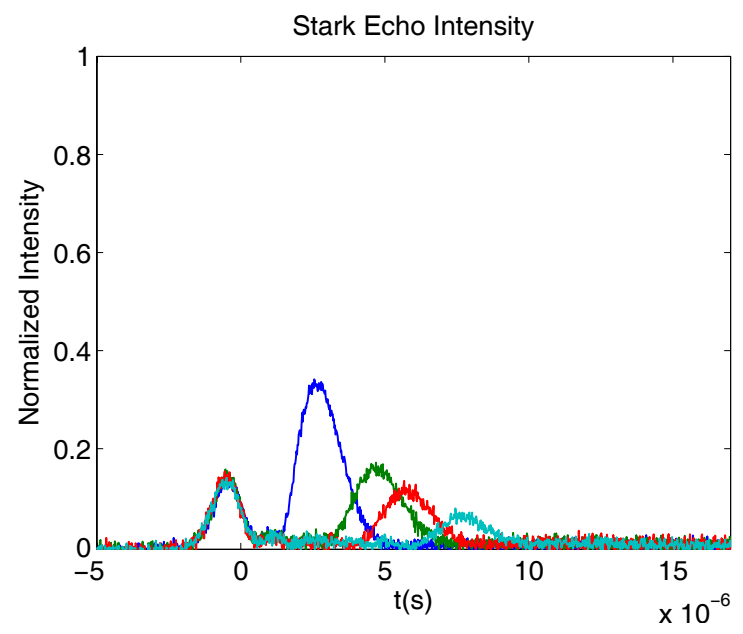

FIG. 20 Transmitted and retrieved pulses for different storage times for an echo based memory.

\section{QUANTUM TELEPORTATION BETWEEN LIGHT AND ATOMS}

\section{A. Quantum Teleportation}

Quantum teleportation is a means for sending quantum states from A to B in a disembodied fashion using two separate channels, a quantum channel connecting A and $\mathrm{B}$ and a classical one. It makes use of entanglement shared via the quantum channel and classical communication. Apart from being one of the most surprising and mind-boggling discoveries in quantum information theory, quantum teleportation has become an essential primitive in quantum computation and quantum communication.

Shortly after the first theoretical layout for quantum teleportation of states of a qubit (Bennett et al. $1993)$ the protocol was extended to states of continuous variables (Braunstein and Kimble, 1998, Vaidman, 1994). Both sorts of protocols were first demonstrated with light, utilizing either probabilistically generated Bell states of photon pairs (Bouwmeester et al. 1997) or deterministically generated EPR beams (Furusawa et al. 1998), both obtained from parametric down conversion. The first teleportation involving massive particles was performed recently in the ion trap experiments at Innsbruck (Riebe et al. 2004) and NIST (Barrett et al. 2004). For a recent review on teleportation of states of continuous variables see (Furusawa and Takei, 2007).

Our focus here is on teleportation protocols involving both matter and light. In such a scenario, quantum states carried by traveling pulses of light are teleported using the quantum interface onto stationary quantum memories realized, for example, by an ensemble of neutral atoms. The first realization of teleportation involving matter and light utilizes the entangled state created via the quantum Faraday - QND interaction of a pulse of light with the collective spin of an atomic ensemble (Sherson et al. 2006c). In this experiment deterministic teleportation with the fidelity higher than any classical state transfer can achieve has been demonstrated. Very recently probabilistic teleportation between light and matter has been also demonstrated using parametric gainand $\Lambda$-type interactions (Chen et al. 2008) and has been extended to entanglement swapping, a concatenation of two teleportation processes (Yuan et al. 2008).

In a nutshell, a teleportation protocol involves three steps: First, an entangled state is created and shared as a resource between two stations (usually termed "Alice" and "Bob"). In the cases considered below, the entanglement between atoms (Bob) and light is generated by sending a strong driving pulse through atoms. As a result of this interaction the forward scattered photons of the orthogonal polarization sent to Alice become entangled with atoms kept by Bob as shown in Fig. 21. The ideal EPR entanglement corresponds to $\Delta\left(X_{A}+X_{L}\right)^{2}=\Delta\left(P_{A}-P_{L}\right)^{2} \rightarrow 0$. Alice also receives another, unknown quantum state described by canonical variables $Y, Q$ sent by a hypothetical sender "Victor", which is to be teleported to Bob. For this, Alice performs a joint measurement of $X_{L}+Y$ and $P_{L}-Q$, called a Bell measurement, on the photonic part of the entangled state that she has received and the unknown quantum state of light to be teleported. This measurement is performed by mixing two light pulses on a beamsplitter 


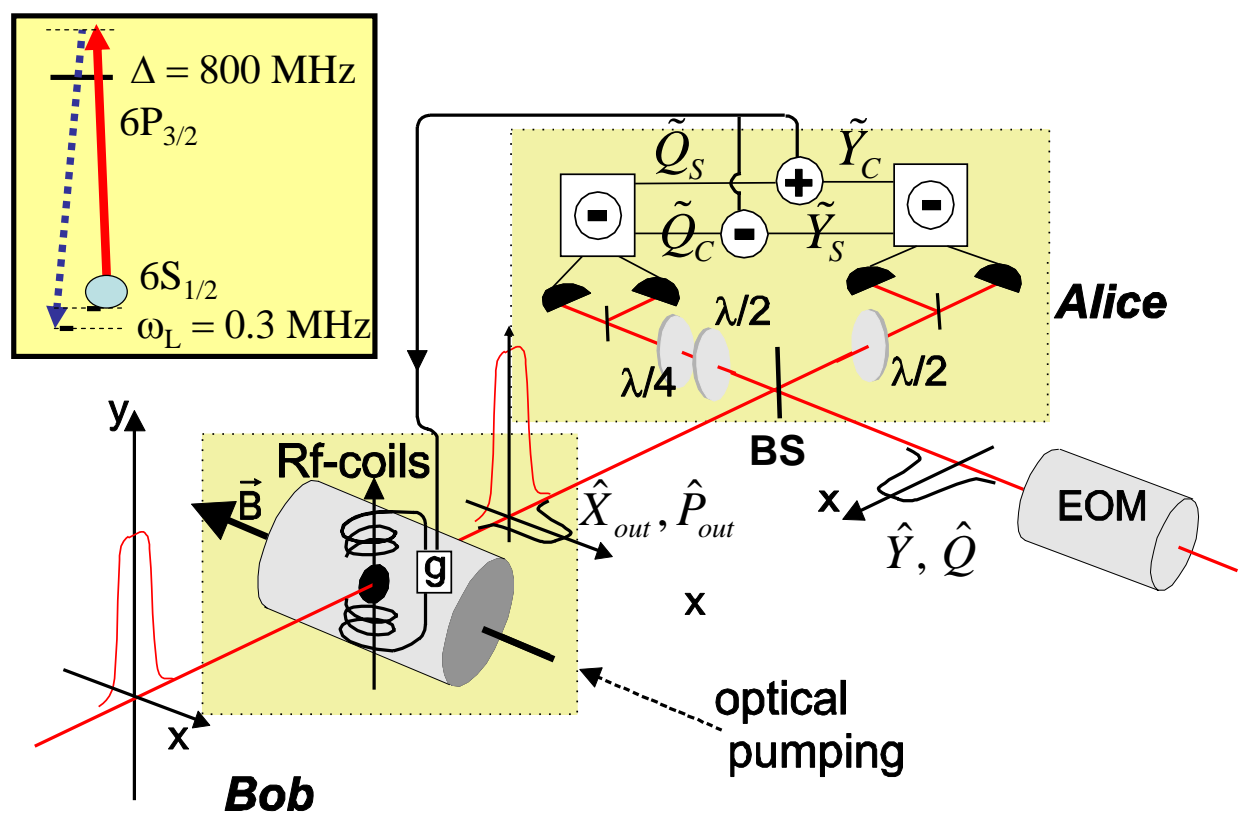

FIG. 21 Experimental setup for the teleportation of light to atoms (Sherson et al. 2006c). Strong pulse y-polarized pulse interacts with atoms and generates an entangled x-polarized mode at the upper sideband frequency (inset). This entangled light is overlapped with the pulse to be teleported on a 50/50 beam splitter BS. The results of the Bell measurements are fed back onto atoms via RF magnetic coils. Detailed comments in the text.

as in Fig. 21. The results of this measurement are communicated to Bob. Bob uses this classical information to perform a correcting operation on his quantum system atoms - by shifting $X_{A \text {,fin }}=X_{A}+\left(X_{L}+Y\right) \rightarrow Y$ and $P_{A, \text { fin }}=P_{A}-\left(P_{L}-Q\right) \rightarrow Q$, thereby recovering the original unknown state.

We see that in the hypothetical case of vanishing EPR variances, first and second moments and thus any Gaussian state (i.e. state with Gaussian wave function or Wigner function) is transmitted perfectly. This in turn implies that any (non-Gaussian) state, including a qubit of the form $\alpha|0\rangle+\beta|1\rangle \in L^{2}(\mathbb{R})$, would be teleported faithfully, as the set of coherent states is a subset of all Gaussian states and provides a basis for the full Hilbert space $L^{2}(\mathbb{R})$ (for the proof of this in the Schrödinger picture and the Wigner function, see Braunstein and van Loock (2005)). In this sense the distinction between teleportation protocols for qubits and continuous variables is superficial. This said, for realistic cases of imperfect teleportation where fidelity is not perfect, performance of each teleportation protocol should be evaluated in detail having in mind a particular application.

If EPR variances do not vanish, as is necessarily the case due to energy restrictions, teleportation will not be perfect, and it is necessary to evaluate the performance of the teleportation. The teleportation is essentially a protocol mapping the state of one system to another similar to the quantum memory protocols. We can therefore use the same figure of merit as discussed in Sec. V.A i.e. for a given class of input states, when is the performance of the protocol better than the best classical protocol.

\section{B. Teleportation based on Faraday interaction}

In Sherson et al. (2006c) the entangled state of light and atoms for teleportation is obtained via the Faraday interaction of light with a single collective atomic spin, precessing in an external magnetic field. The relevant level scheme is shown in Fig. 4 and in the inset to Fig. 21. The situation is described by the Hamiltonian and Maxwell-Bloch equations given in section II.E. In this case, the Hamiltonian does not fulfil the QND criteria (Holland et al., 1990, Poizat et al. 1994) because the Faraday interaction $H_{F}$, c.f. Eq. (34), does not commute with the free Hamiltonian 63 describing Zeeman splitting of ground states. The resulting Maxwell-Bloch equations (65) are integrated by Hammerer et al. (2005a, 2006) and the solutions are again conveniently expressed in terms of experimentally measurable cosine and sine 
modulation modes 66],

$$
\begin{aligned}
X_{A, \text { out }}= & X_{A, \text { in }}+\frac{\kappa}{\sqrt{2}} P_{L_{c}, \text { in }}, \\
P_{A, \text { out }}= & P_{A, \text { in }}+\frac{\kappa}{\sqrt{2}} P_{L_{s}, \text { in }}, \\
P_{L_{c}, \text { out }}= & P_{L_{c}, \text { in }}, \\
X_{L_{c}, \text { out }}= & X_{L_{c}, \text { in }}+\frac{\kappa}{\sqrt{2}} P_{A, \text { in }} \\
& \quad+\left(\frac{\kappa}{2}\right)^{2} P_{L_{s}, \text { in }}+\frac{1}{\sqrt{3}}\left(\frac{\kappa}{2}\right)^{2} P_{L_{s, \text { back }}, \text { in }}, \\
P_{L_{s}, \text { out }}= & P_{L_{s}, \text { in }}, \\
X_{L_{s}, \text { out }}= & X_{L_{s}, \text { in }}-\frac{\kappa}{\sqrt{2}} X_{A, \text { in }} \\
& \quad-\left(\frac{\kappa}{2}\right)^{2} P_{L_{c}, \text { in }}-\frac{1}{\sqrt{3}}\left(\frac{\kappa}{2}\right)^{2} P_{L_{c, \text { back }}, \text { in } .}
\end{aligned}
$$

The terms proportional to $\kappa^{2}$ are a new feature, specific for this setup, and represent atom-mediated back-action of light onto itself. This effect involves the previously defined cosine and sine modes as well as yet another pair of canonical independent "back-action modes" $P_{L_{c(s), b a c k} \text {, in }}$ which can be treated as vacuum noise operators. To see that this interaction can be used for teleportation let us inspect the EPR type correlations between the atomic mode $X_{A \text {, out }}, P_{A \text {, out }}$ and the light mode of the upper sideband $X_{+}, P_{+}$with the frequency $\omega+\omega_{L}$

$$
\begin{aligned}
& X_{L_{+}, \text {out }}=\frac{1}{\sqrt{2}}\left(X_{L_{s}, \text { out }}-P_{L_{c}, \text { out }}\right), \\
& P_{L_{+}, \text {out }}=\frac{1}{\sqrt{2}}\left(X_{L_{c}, \text { out }}+P_{L_{s}, \text { out }}\right) .
\end{aligned}
$$

One easily finds

$$
\begin{array}{r}
\Delta\left(X_{A, \text { out }}+X_{L_{+}, \text {out }}\right)^{2}=\Delta\left(P_{A, \text { out }}-P_{L_{+}, \text {out }}\right)^{2} \\
=\frac{1}{4}\left[1+\left(1-\frac{\kappa}{2}\right)^{2}\right]^{2}+\frac{1}{6}\left(\frac{\kappa}{2}\right)^{4} \gtrsim 0.33
\end{array}
$$

where the lower bound is achieved for $\kappa \simeq 1.48$. The given modes of light and atoms are in an entangled state which can be used for teleportation.

The state to be teleported is encoded in the lower sideband $\omega-\omega_{L}$ with respect to the carrier frequency (Fig. 21), expressed in terms of measurable cosine and sine modulation modes as

$$
Y=\frac{1}{\sqrt{2}}\left(Y_{\mathrm{s}}+Q_{\mathrm{c}}\right), \quad Q=-\frac{1}{\sqrt{2}}\left(Y_{\mathrm{c}}-Q_{\mathrm{s}}\right),
$$

where $[Y, Q]=i$. The Bell measurement of the commuting observables after combining the entangled and the to-be-teleported states yields

$$
\begin{array}{ll}
\tilde{X}_{\mathrm{c}}=\frac{1}{\sqrt{2}}\left(X_{L_{c}, \text { out }}+Y_{\mathrm{c}}\right), & \tilde{X}_{\mathrm{s}}=\frac{1}{\sqrt{2}}\left(X_{L_{s}, \text { out }}+Y_{\mathrm{s}}\right), \\
\tilde{Q}_{\mathrm{c}}=\frac{1}{\sqrt{2}}\left(P_{L_{c}, \text { out }}-Q_{\mathrm{c}}\right), & \tilde{Q}_{\mathrm{s}}=\frac{1}{\sqrt{2}}\left(P_{L_{s}, \text { out }}-Q_{\mathrm{s}}\right) .
\end{array}
$$

Conditioned on these results the atomic state is then displaced in order to get in the ensemble average a final state

$$
\begin{gathered}
X_{A, \text { fin }}=X_{A, \text { out }}+\tilde{X}_{\mathrm{s}}-\tilde{Q}_{\mathrm{c}}=\left(X_{A, \text { out }}+X_{L_{+}, \text {out }}\right)+Y \\
P_{A, \text { fin }}=P_{A, \text { out }}-\tilde{X}_{\mathrm{c}}-\tilde{Q}_{\mathrm{s}}=\left(P_{A, \text { out }}-P_{L_{+}, \text {out }}\right)+Q .
\end{gathered}
$$

In the hypothetical case of vanishing EPR variances of $\left(X_{A, \text { out }}+X_{L_{-}, \text {out }}\right)$ and $\left(P_{A \text {, out }}-P_{L_{-}, \text {out }}\right)$ atoms would correctly display the statistics of the $Y, Q$ mode, reproducing any input state (coherent, Fock, etc) as desired. For the given minimal EPR variances of 0.33 teleportation will not be perfect, but still below the classical limit corresponding to the EPR variance of 1 , that is to two units of added vacuum noise. This discussion still ignores atomic decay and light absorption. It can be included as discussed in section II.C

The experimental implementation of this teleportation protocol by Sherson et al. (2006c) was performed with a new generation of paraffin coated cells filled with Caesium similar to those shown in Fig. 8. Atoms are initially prepared in a coherent spin state by a $4 \mathrm{msec}$ circularly polarized optical pumping pulse propagating along the direction of the magnetic field, into the sublevel $F=4, m_{F}=4$ of the ground state (insert in Fig. 21). Then an entangled light-atoms state is generated by sending a strong pulse polarized along the $y$ axis (Fig. 21). The initially vacuum state of the $x$ polarization of this pulse is populated after the interaction with the field $X_{L, \text { out }}, P_{L, \text { out }}$ which is entangled with the atomic variables $X_{A, \text { out }}, P_{A \text {,out }}$ according to (85). (Compared to the theoretical derivation of the Faraday interaction in Sec. II the polarizations of the classical and quantum fields are interchanged. This is of minor importance since it merely swaps the vertical and diagonal transitions in Fig. 2.)

The nearly optimal value of the coupling constant $\kappa \approx 1$ was achieved with $4 \times 10^{13}$ photons in the strong pulse with the duration of $1 \mathrm{msec}$ and a crossection of $4.4 \mathrm{~cm}^{2}$ detuned by $825 \mathrm{MHz}$ and a number of atoms on the order of $10^{12}$ corresponding to the Cs temperature of $25^{\circ} \mathrm{C}$. At Alice's location the mode of light entangled with atoms is combined with the input pulse to be teleported on a 50/50 beamsplitter BS (Fig. 21). The strong pulses which travels along with the entangled quantum field conveniently serves as the local oscillator for the polarization homodyne measurements of the Stokes operators $S_{y}$ and $S_{z}$ performed at two outputs of BS. The output $\cos \left(\omega_{L} t\right)$ and $\sin \left(\omega_{L} t\right)$ components of the photocurrent are processed by the lock-in amplifiers to produce the feedback signals $\widetilde{Q}_{c, s}, \widetilde{Y}_{c, s}$. The two feedback signals at $\omega_{L}=322$ $\mathrm{kHz}$ phase shifted by $\pi / 2$ with respect to each other are fed into the RF magnetic coils surrounding the atoms with a variable electronic gain. The gain is chosen so that the atomic variables are shifted by one vacuum unit if the input light mode contains one vacuum unit of excitation. This condition corresponds to the "unity gain" teleportation.

To prove the success of the teleportation protocol a strong verifying pulse reads out the atomic operators 
(collective spin projections). The same homodyne polarization measurement setup is used for that. An example of the atomic state readout is shown in Fig. 22. The gain of 0.95 is found from the slope of the linear fit to the data, whereas the variances of the final atomic state after teleportation $\Delta X_{A, \text { out }}=\Delta P_{A, \text { out }}=1.2$ are found from the variance of the distribution of the data. These variances are below the classical teleportation limit corresponding to three units of vacuum noise, that is to $3 / 2$. Accordingly the fidelity of the teleporation calculated as

$$
\begin{aligned}
F=\left(\bar{n}(1-g)^{2}+1 / 2+\Delta X_{A, \text { out }}^{2}\right)^{-1 / 2} \\
\left(\bar{n}(1-g)^{2}+1 / 2+\Delta P_{A, \text { out }}^{2}\right)^{-1 / 2}
\end{aligned}
$$

for a distribution of coherent states with a width of $\bar{n}$ is greater than the best classical fidelity. Indeed the fidelities of, e.g., $F=0.60 \pm 0.02$ and $F=0.58 \pm 0.02$ have been obtained for sets of input states with $\bar{n}=5$ and $\bar{n}=20$ respectively whereas best classical fidelities for these cases are 0.54 and 0.51 .

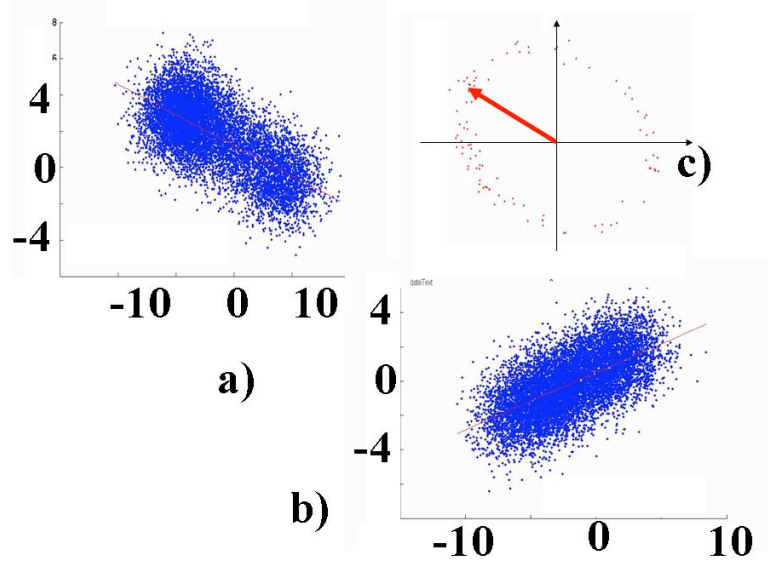

FIG. 22 Teleportation results (Sherson et al., 2006c). a) and b) Two canonical operators for the verifying pulse plotted as a function of the corresponding canonical operators of the input pulse for $2 \cdot 10^{3}$ realizations (vacuum units). The slope which is close to $1 / 2$ should be multiplied by 2 to account for the attenuation of the verifying pulse on the beam splitter. From the variance of the distribution along the vertical axis the higher than classical fidelity is obtained. c) A set of input states with $\langle n\rangle=5$ and random phases.

As shown in the Supplementary Notes to Sherson et al. (2006c) and in Hammerer et al. (2006) the knowledge of $\Delta X_{A, \text { out }}, \Delta P_{A, \text { out }}$ for the teleportation of the coherent states corresponds to the complete knowledge of the teleportation map and allows to calculate the fidelity of the qubit teleportation as

$$
\begin{aligned}
& F_{q}=\left(6+16 s^{2}+\right. 24 s^{4}+4(g-1)\left(1-2 s^{2}\right)+ \\
&\left.(g-1)^{2}\left(1-6 s^{2}\right)\right) / 6\left(1+2 s^{2}\right)^{3}
\end{aligned}
$$

where $s^{2}=2 \Delta X_{A, \text { out }}^{2}-1$ and equal quality of mapping for the two canonical operators is assumed $\Delta X_{A \text {, out }}=$
$\Delta P_{A, \text { out }}$. Direct demonstration of the qubit teleportation under the conditions of Sherson et al. (2006c) has not been possible due to the absence of a light qubit source with the pulse duration of 1 msec. As shown in Sherson et al. (2006c), in principle a qubit fidelity of $F_{q}=0.74$ is achievable for $\kappa=1$.

The protocol can in principle be improved by properly taking back-action modes, treated here simply as noise terms, into account. This is to a large extent a question of detector bandwidth and improved post processing of photocurrents. In Hammerer et al. (2005a) it is shown that in this way the fidelity can be increased up to $80 \%$ corresponding to half a unit of added vacuum noise. This noise stems from the initial vacuum fluctuations of light before light-atom interaction which can in turn be reduced by using a squeezed light for entanglement with atoms. One ends up with a similar situation as in the standard protocol (Braunstein and Kimble, 1998, Vaidman, 1994): the quality of teleportation is in the end limited by the amount of available squeezing, which emerges here once more to be an irreducible resource (Braunstein, 2005).

A number of alternative proposals for deterministic teleportation involving light and atomic ensembles have been suggested. Horoshko and Kilin (2000); Mišta and Filip (2005) study the application of the state of light and atoms created in a QND interaction, cf. (40), as a resource for teleportation and show that the use of squeezing of light and atoms as well as unbalanced beam splitters in the Bell measurement can improve the fidelity. Teleporation of states of light to atoms, based on entanglement between motional degrees of freedom of a BoseEinstein condensate and light has been suggested by Cola et al. (2004); Paris et al. (2003).

\section{ERRORS AND FIDELITY FOR DIFFERENT INTERFACES}

Despite many impressive successes the unconditional fidelity and/or efficiency of the quantum interfaces demonstrated so far does not exceed $70 \%$ and in many cases is at the level of $20 \%$. Several factors, some more fundamental and some more technical contribute to this.

Scaling with optical depth. In the theory section we have shown that spontaneous emission can be avoided for the optical depth $d \gg 1$. Here we discuss the errors due to a finite $d$ for different approaches. In the entanglement section we have shown that the single ensemble squeezing $\Delta P_{A}^{2}$ or the two ensemble correlation $\Delta_{E P R}$ is $\sim 1 / \sqrt{d}$. This limit, however, depends on the exact decay mechanism, and a better scaling of single ensemble squeezing $1 / d$ can be achieved if the QND interaction through phase shift measurements using two closed transitions (Sec. II.G), is used, since in this case spontaneous emission does not add noise to $P_{A}$. 
For a quantum memory the inefficiency of storage with the beam splitter interaction (Raman or EIT) scales as $1 / d$ if suitably shaped spatial mode functions are used. If storing or reading out the spatially symmetric mode is preferable, as, in the repeater case where the probabilistic entanglement is generated in this mode (Sec. IV.C), the scaling is again weak, $1 / \sqrt{d}$ (Gorshkov et al. 2007a c). However, a much better performance can be achieved with ensembles in optical cavities Gorshkov et al. 2007b), where the scaling is $1 / \mathcal{F} d(\mathcal{F}$ is the cavity finesse). The protocols based on the Faraday interaction naturally couple to the symmetric modes and may be better suited for this mode than the beam splitter interaction. These memory protocols have a coupling constant $\kappa^{2} \sim 1$, which yields $\sim 1 / d$ or $\sim \log (d) / d$ (Sherson et al. 2006a) error due to spontaneous emission .

Different protocols are also characterized by different kinds of errors. The protocols based on the single pass QND - Faraday interaction have variable gain, faithfully reproduce mean values of the input states, and thus give good fidelity over a large phase space. The error in the beam splitter protocols corresponds to a loss on a beam splitter and thus gives bad fidelity for states with a large amplitude. On the other hand the low fidelity beam splitter (Raman and EIT) protocols usually add only vacuum noise, whereas single pass QND-based protocols add multi-photon errors. Which kind of error is less harmful depends on a particular application. For instance Brask and Sørensen (2008) show that memories based on the single pass QND - Faraday interaction are not very well suited for the DLCZ-repeater protocol (Duan et al. 2001), because the repeater protocol is specifically designed to correct only for the photon loss errors. A full evaluation of the performance thus depends on the particular application one has in mind.

Inhomogeneous broadening Another source of possible errors is inhomogeneous broadening of the optical transitions. Solid state systems have a strong inhomogeneous broadening because each of the emitters sits in a slightly different environment, while inhomogeneous broadening in room temperature atomic ensembles is due to the Doppler broadening of the atomic lines. In the theoretical derivation we only assumed a homogeneous broadening of the optical transitions (the photon echo approach discussed in the memory section is a notable exception). One therefore cannot just replace the optical depth $d$ appearing in the formulas derived in the theory section by the measured optical depth in the presence of inhomogeneous broadening.

A detailed study of the effect of inhomogeneous broadening for a quantum memory using the beam splitter interaction is presented by Gorshkov et al. (2007d) who have shown that even far off-resonance the inhomogeneous broadening of the line still plays a role. The reason is that the strong field leads to a considerable ACStark shift of the ground state $|1\rangle$ even far off resonance.
The inhomogeneous broadening introduces energy shifts which are different for each atom and thus causes decoherence of the collective states. As a result the effect of inhomogeneous broadening is as severe off resonance as it is on resonance. Hence the total inefficiency of the memory protocol has two contributions. The first is the spontaneous emission which scales as $1 / d_{\text {hom }}$, where $d_{\text {hom }}$ is the optical depth of the ensemble without the broadening. The second contribution is from the inhomogeneous broadening and scales as $1 / d_{\text {inhom }}^{2}$, where $d_{\text {inhom }}$ is the actual optical depth in the presence of broadening (this scaling assumes that the broadened lines fall off sufficiently fast; for other profiles, e.g., Lorentzian, the scaling is $1 / d_{\text {inhom }}$ ). If one increases the length of the sample the system will eventually be dominated by the $1 / d_{\text {hom }}$ contribution and thus behaves as if it were homogeneously broadened.

For the Faraday interaction the situation is different. The Faraday interaction is only employed far off resonance, and unlike the case of the beam splitter interaction the AC-stark shift does not cause decoherence. The strong classical light couples to both ground states and shifts them by the same amount. As a result the spin dynamics is not affected by a difference in the level shifts, and the Faraday interaction becomes insensitive to the inhomogeneous broadening for detunings much larger than the hyperfine structure and Doppler width.

Atomic motion As discussed in Sec. III atoms moving in and out of the beam may severely effect the performance of the interface, but for the Faraday interaction a strong suppression of this effect is achieved in the experiments by Julsgaard et al. (2001, 2004a); Sherson et al. (2006c) because the light beams cover most of the cell volume and the interaction time is much longer than the atomic transient time of flight. This suppression is, however, not perfect. As analyzed in detail in Sherson et al. (2006b) the fact that atoms go in and out of the interaction volume leads to extra spin noise which should be accounted for when the projection noise level is being established.

Atomic collisions For atomic gasses it is also important to consider the effect of atomic collisions. For experiments with paraffin coated cells atom-atom collisions contribute up to $20-40 \mathrm{~Hz}$ to the ground state decoherence which is usually much less than the contribution from collisions with walls for $25 \mathrm{~mm}$ cubic cells (Sherson et al. 2006b). In experiments where a buffer gas is used to suppress atomic motion, the alkali atoms - buffer gas collisions often have little effect on the ground state coherence of the atoms and the memory time. The buffer gas does, however, change the dynamics during the interaction with the light. The homogeneous broadening due to collisions with the buffer gas can be included in the theory by modifying the homogenous line width $\gamma$. At the same time collisions change the velocity of the atoms and 
thereby their Doppler shift. The collisions thus change the phase spreading which causes decoherence of the collective states from being ballistic to diffusive, and this reduces the inefficiency from the inhomogeneous broadening.

For the write stage of the probabilistic entanglement protocol a different effect related to the collisional broadening has been observed by Manz et al. (2007) and has been discussed theoretically in a different context (Childress et al. , 2005). The collisions with buffer gas atoms cause the alkali atoms to emit photons at the resonance frequency of the atoms rather than at the anti-Stokes frequency. To observe the entanglement it is thus necessary to filter out these incoherent photons with a frequency filter. This effect, however, has little consequences for the other protocols.

Geometry of the ensemble. In most cases the two ground states of the ensemble are nondegenerate, so there is an additional phase factor $\exp \left[i\left(k_{0}^{\prime}-k_{0}\right) z\right]$ associated with the difference of the $k$ vectors for the classical and quantum fields. This phase can be absorbed into the definition of the mode functions $u_{m}(\vec{r}, t)$ in $(29)$ and $\left.\sqrt{32}\right)$, and does not play a role for the beam splitter and parametric gain interaction applied separately. However, if one reads out the memory in the backward direction, which is sometimes advantageous, or combines the two protocols, the atomic operators should be redefined and this phase can have a detrimental effect (André, 2005: Duan et al., 2002, Gorshkov et al., 2007c; Surmacz et al., 2007). For the Faraday interaction on the other hand, the mode functions must have a constant phase and this means that the atomic ensemble must be much smaller than the wavelength corresponding to the ground state splitting. In addition the Fresnel number corresponding to the shape of the atomic ensemble must be large in order to stay within a single transverse spatial mode approximation.

Deviation from a two-level ground state model. When the ground state level used for the interface is magnetically degenerate with more than two states and the detuning of the strong field is not sufficiently larger than the hyperfine splitting of the excited state, the interaction of the Faraday type ( $a_{1}$ vector term in Sec. II.D) is contaminated with the Raman interaction ( $a_{2}$ tensor term). This leads to extra noise, as well as to additional decoherence which is proportional to the interaction constant $\kappa^{2}$ and hence limits the fidelity of the interface. Various aspects of this effect have been considered in Julsgaard (2003) Kupriyanov et al. (2005), and Sherson et al. (2006b), as well as in Geremia et al. (2006). This issue is at the moment the major limitation for the fidelity of the Faraday-based protocols and is under active investigation.

The beam splitter interaction (EIT) experiments mostly use two ground hyperfine levels. Magnetic de- generacy leads to imperfections which can be reduced by careful optical pumping and polarization filtering Choi et al. 2008).

\section{OUTLOOK}

The light-matter quantum interface, a term coined in the end of $90 \mathrm{~s}$, is one of the pillars of the field of Quantum Information Processing and Communication (QIPC) (Zoller et al. 2005). In less than a decade since the first demonstrations of a quantum interface between light and an atomic ensemble the ensemble approach has become one of the most active areas of research in the field. The directions which seem most promising at the moment are discussed in this review: QND - Faraday interaction, EIT, and photon echo.

Both fundamental and application driven aspects are obvious within this approach. One of the interesting fundamental issues concerns the multi-particle entanglement necessarily present in the ensemble-based approach. At the same time the interface is a kind of a quantum channel, hence its relation to the theory of quantum channel capacity should be explored in the future. This issue is connected to the fidelity of the interface since it is known that a quantum channel with $F>2 / 3$ for coherent states has a non-zero quantum capacity (Grosshans and Grangier, 2001, Wolf et al., 2007). Quantum interface also allows for storing optimal quantum clones of a state of light as proposed by Fiurǎśek et al. (2004). Another feature which is intrinsic for the ensembles of atoms is the spatially multi-mode capacity paving the road towards "quantum holograms" (Surmacz et al. , 2007; Vasilyev et al. . 2008). The first experimental storage of classical images via the EIT approach have recently been demonstrated (Shuker et al. 2007, Vudyasetu et al., 2008).

Long distance quantum communication is one of the most actively pursued applications of the interface at the moment. It is based on the combination of probabilistic entanglement generation and deterministic entanglement swapping - a quantum repeater with atomic ensembles Duan et al. (2001) - which may serve as the basis for a "quantum internet" (Kimble, 2008).

Advanced architectures for quantum computing may be enabled by highly efficient photon-based connections between small scale atomic processing nodes (Jiang et al. 2007). An interesting direction in this respect is the combination of photon counting and QND-Faraday continuous variable measurement techniques. It allows to combine the best of the two worlds: the high efficiency of the homodyne measurement and the non-Gaussian states, such as Schrödinger cat states which can be generated by photon counting (Genes and Berman, 2006; Massar and Polzik, 2003). A major breakthrough in this respect may come with the development of highly efficient photon counters and photon number resolving detectors Achilles et al. 2004, Waks et al. 2006).

For new applications of quantum interfaces a major 
challenge will be for the experimentalists to improve the fidelity and efficiency of the interface, as well as for theorists to find protocols where atomic memories with the fidelity and efficiency at the level of $90-95 \%$ - the likely levels to be achieved within the next few years - can help to achieve goals impossible with classical memories.

Besides the fiducial write- and read-processes and long storage times, a quantum interface between light and matter will likely have to show yet another key element: the possibility to process stored quantum information and to allow for quantum logical gate operations for active entanglement purification and error correction (Dür and Briegel, 2007). Theoretical studies of the requirements on gate operations in quantum repeater architectures have been performed in great detail by Briegel et al. (1998); Dür et al. (1999); Hartmann et al. (2007) and Dorner et al. (2008); Klein et al. (2006) recently investigated the usage of decoherence free subspaces in quantum communication. Proposals for ensemble-based implementations unifying an efficient light matter interface, stable quantum memory and reliable small scale quantum processors are rare. A number of theoretical studies suggest gate operations on stored collective excitations via a Rydberg blockade mechanism (Brion et al., 2007; Lukin et al., 2001; Petrosyan and Fleischhauer, 2008), via an EIT enhanced optical nonlinearity (André et al. 2005, Lukin and Imamoglu, 2001; Ottaviani et al. 2003, Wang et al. | 2006) or in hybrid systems, where a Cooper pair box serves as a saturable, nonlinear element (Rabl et al. 2006). Initial experiments along those lines are in progress.

Efficient ensemble-based quantum memories and matter-light interfaces, small scale quantum processors for error correction, repeaters, possibly satellite based quantum communication (Aspelmeyer et al. 2003 , Pfennigbauer et al., 2005), and even hybrid systems (Hammerer et al. 2008) - are the goals of today. The research performed towards this end is of both fundamental interest for our understanding of quantum physics and of technological importance. Its highly interdisciplinary character encompasses a broad spectrum of fields in physics as well as in computer science and information theory.

\section{Acknowledgments}

We are grateful to our colleagues with whom we have had collaboration and many useful discussions on the subject of quantum interfaces over the past years. In particular we would like to thank J. I. Cirac, N. Cerf, M. Fleischhauer, J. Fiurăśek, A. V. Gorshkov, A. Kuzmich, M. Lukin, S. Massar, J. H. Müller, H. J. Kimble, K. Mølmer, R. Walsworth, and P. Zoller. E.P. would like to especially thank the enthusiastic and talented members of his experimental team for whom the quantum interface has been a buzz word for the last decade. This work has been supported by the Danish National Research Foundation, by the EU programs COVAQUIAL, QAP,
HIDEAS, COMPAS, CONQUEST and EuroSQUIP and by the Austrian FWF through SFB F15.

\section{APPENDICES}

\section{A. Adiabatic Elimination}

The effective ground state Hamiltonian can be obtained by adiabatic elimination. We start with the dipole Hamiltonian $H_{\text {int }}=\vec{E} \cdot \vec{D}$. For magnetic sub-levels $\left\{\left|g_{m}\right\rangle\right\}$ all matrix elements of the ground state dipole operator vanish $\left\langle g_{m}|\vec{D}| g_{m^{\prime}}\right\rangle=0$, and similarly for the excited state $\left\langle e_{m}|\vec{D}| e_{m^{\prime}}\right\rangle=0$. Introducing positively and negatively oscillating components $\vec{D}=\vec{D}^{(+)}+\vec{D}^{(-)}$and $\vec{E}=\vec{E}^{(+)}+\vec{E}^{(-)}$we obtain in the rotating wave approximation

$$
H_{\mathrm{int}}=\vec{E}^{(-)} \cdot \vec{D}^{(+)}+\vec{D}^{(-)} \cdot \vec{E}^{(+)},
$$

where the first (second) term describe up (down) transitions. Expanding the Hamiltonian we obtain

$$
H_{\mathrm{int}}=\sum_{m, m^{\prime}} \vec{E}^{(-)} \cdot \vec{D}_{m m^{\prime}}^{(+)}\left|g_{m}\right\rangle\left\langle e_{m^{\prime}}\right|+\text { H.C. }
$$

Using the Hamiltonian $H_{\mathrm{A}}+H_{\text {int }}$ we obtain

$$
\begin{aligned}
& \frac{d}{d t}\left|g_{m}\right\rangle\left\langle e_{m^{\prime}}\left|=-i \Delta_{m^{\prime}}\right| g_{m}\right\rangle\left\langle e_{m^{\prime}}\right| \\
& \quad-i \vec{E}^{(+)} \sum_{m^{\prime \prime}}\left(\vec{D}_{m^{\prime}, m^{\prime \prime}}^{(-)}\left|g_{m}\right\rangle\left\langle g_{m^{\prime \prime}}\left|-\vec{D}_{m^{\prime \prime}, m}^{(-)}\right| e_{m^{\prime \prime}}\right\rangle\left\langle e_{m^{\prime}}\right|\right)
\end{aligned}
$$

Here we ignore the Langevin noise operators associated with the spontaneous emission. For weak excitation (far below saturation) we can neglect the excited state operator $\left|e_{m^{\prime \prime}}\right\rangle\left\langle e_{m^{\prime}}\right|$. Next, assuming the dynamics is slow compared to the detuning $\Delta$, we ignore the left hand side compared to the first term on the right hand side. These approximations are valid provided that $\vec{E}^{(+)} \cdot \vec{D}_{m, m^{\prime}}^{(-)} \ll \Delta_{m^{\prime}}$. In this limit we obtain

$$
\left|g_{m}\right\rangle\left\langle e_{m^{\prime}}\left|\approx-\frac{\vec{E}^{(+)} \cdot \vec{D}_{m^{\prime}, m^{\prime \prime}}^{(-)}}{\Delta_{m^{\prime}}}\right| g_{m}\right\rangle\left\langle g_{m^{\prime \prime}}\right| .
$$

To obtain an effective ground state Hamiltonian we substitute the expression (93) back into the Hamiltonian $H_{\mathrm{A}}+H_{\text {int }}$. Note that we have to choose normal ordering of the operators in Eq. (91) when we insert Eq. (93). A detailed discussion of this issue may be found in Barnett and Radmore (1997). Secondly, since the terms in $H_{\mathrm{A}}$ involve the excited state population one could be tempted to ignore it since the population is proportional to $1 / \Delta_{m}^{2}$. On the other hand there is also a factor $\Delta_{m}$ in front of this term, so that in total this is on the order of $1 / \Delta_{m}$ and we have to include it. To treat this term we introduce any intermediate state $\left|g_{0}\right\rangle$

$$
\left|e_{m^{\prime}}\right\rangle\left\langle e_{m^{\prime}}|=| e_{m^{\prime}}\right\rangle\left\langle g_{0}|\cdot| g_{0}\right\rangle\left\langle e_{m^{\prime}}\right|
$$


and substitute the result (93) for $\left|g_{0}\right\rangle\left\langle e_{m^{\prime}}\right|$. By doing this we arrive at the effective Hamiltonian in Eq. (25).

\section{B. Three dimensional Hamiltonians}

In the main text we present the general expression (27) for the full three dimensional Hamiltonian. In this appendix we discuss the 3D Hamiltonians for the three model system, beams splitter, parametric gain, and Faraday interaction.

First we consider the atomic beam splitter interaction in 2(a). We assume that the state $|0\rangle$ is coupled to an excited state by a classical field, so that $\vec{E}(\vec{r})=\langle\vec{E}(\vec{r})\rangle$. Inserting the expression for the quantized electric field in Eq. 12 we obtain

$$
\begin{aligned}
H_{B S}= & \int d^{3} \vec{r}\left[\frac{-|\Omega(\vec{r}, t)|^{2}}{4 \Delta} a_{A}^{\dagger}(\vec{r}) a_{A}(\vec{r})\right. \\
& -\frac{|g(\vec{r})|^{2}}{\Delta} \sum_{m}\left|u_{m}\left(\vec{r}_{\perp} ; z\right)\right|^{2} a_{L, m}^{\dagger}(z) a_{L, m}(z) \\
& -\sum_{m}\left(\frac{g^{*}(\vec{r}) \Omega(\vec{r}, t)}{2 \Delta} u_{m}^{*}\left(\vec{r}_{\perp} ; z\right)\right. \\
& \left.\left.\times a_{L, m}^{\dagger}(z) a_{A}(\vec{r})+\text { H.C. }\right)\right],
\end{aligned}
$$

where the coupling constant $g(\vec{r})$ and resonant Rabi frequency are defined as in Eq. (31) except with $z$ replaced by $\vec{r}$. The first term in this Hamiltonian is the AC-Stark of the ground state caused by the classical field, the second term is the change in the index of refraction that the quantum field experiences, and the last term represents the exchange of excitation between the light and the ground state coherence. We have here ignored the small AC-Stark shift caused by the weak quantum field.

The difference between the beam splitter interaction and the parametric gain in Fig. 2(b), is that now the atoms are prepared in the other atomic ground state. To obtain the parametric Hamiltonian we thus make a replacement $a_{A}(\vec{r}) \leftrightarrow a_{A}^{\dagger}(\vec{r})$. In addition the AC-Stark shift has the opposite sign. The Hamiltonian thus reads

$$
\begin{aligned}
H_{G}= & \int d^{3} \vec{r}\left[\frac{|\Omega(\vec{r}, t)|^{2}}{4 \Delta} a_{A}^{\dagger}(\vec{r}) a_{A}(\vec{r})\right. \\
& -\sum_{m}\left(\frac{g^{*}(\vec{r}) \Omega(\vec{r}, t)}{2 \Delta} u_{m}^{*}\left(\vec{r}_{\perp} ; z\right)\right. \\
& \left.\left.a_{L, m}^{\dagger}(z) a_{A}^{\dagger}(\vec{r})+\text { H.C. }\right)\right] .
\end{aligned}
$$

There is no index of refraction here since now the quantum field couples to the almost empty transition. On the other hand the classical field now sees the atomic population, and we should include the index of refraction in the propagation of the classical light, which enters through the phase of $\Omega$.

For the Faraday interaction we assume a strong $x$ polarized classical field, and consider the quantum field in the $y$-polarization as shown in Fig. 22(c). There are now two paths which may lead to creation of a photon: photon creation (or absorption) may appear both through the creation and annihilation of an atomic excitation. As discussed in the main text the Faraday interaction is a combination of the two Hamiltonians in Eqs. 95) and (96). For the $1 / 2-1 / 2$ transition depicted in Fig. 2 , however, the two paths leading to the creation of a photon have opposite signs due to the Clebsch-Gordon coefficients. The coupling constant $g$ is defined for circularly polarized light, so we should include a factor of $\sqrt{2}$ coming from the expansion of the $y$-polarized quantum field in the circular polarization basis. The Hamiltonian is then given by $H_{F}=\left(H_{B S}-H_{G}\right) / \sqrt{2}$.

For the spin $1 / 2$ system classical and quantum fields experience the same index of refraction, so we can remove the index of refraction from $H_{B S}$ if we also ignore it for the classical field. An important result for this Hamiltonian is that the AC-Stark shift of the ground state disappears, because the classical field shifts the two atomic states by the same amount.

\section{Propagation equations for light}

The light Hamiltonian (for a single transverse mode) is given by

$$
H_{L}=\sum_{k}|k| c a_{k}^{\dagger} a_{k}
$$

where the sum is over all the longitudinal wave vectors. We derive the time evolution of the operator $a_{L}(z)$ defined in Eq. 10. If we assume that the modes contributing to the slowly varying operator $a_{L}(z)$ are centered around the large positive value $k_{0}$, we can ignore the absolute value of the wave vector. Combining the time derivative caused by the explicit time dependence introduced in 10 with the time evolution caused by $H_{L}$ we find the Heisenberg equation of motion

$$
\frac{\partial a_{L}}{\partial t}=i \omega_{0} a_{L}-i\left[a_{L},\left(H_{\mathrm{L}}+H_{\mathrm{int}}\right)\right]=-c \frac{\partial}{\partial z} a_{L}-i\left[a_{L}, H_{\mathrm{int}}\right] .
$$

To simplify the equations we introduce a rescaled time variable by defining new operators $\tilde{a}_{L}(z, \tau)=a_{L}(z, t=$ $\tau+z / c)$ and $\tilde{a}_{A}(z, \tau)=a_{A}(z, t=\tau+z / c)$. The propagation equation for the light can then be simplified by using

$$
\left(\frac{\partial}{\partial t}+c \frac{\partial}{\partial z}\right) a_{L}(z, t=\tau+z / c)=c \frac{\partial}{\partial z} \tilde{a}_{L}(z, \tau) .
$$

We also take the classical field to be moving in the positive $z$-direction, in which case the Rabi-frequency is $\Omega(z, t)=\Omega(t-z / c)=\Omega(\tau)$. The equations of motion in 
the main text always use this rescaled time and we omit the tilde on the operators and simply denote the rescaled time $\tau$ by $t$.

For the parametric gain the $z$ dependence of the Rabi-frequency does not disappear completely from the equations of motion since we should include the $\exp \left(i \int d z^{\prime}\left|g\left(z^{\prime}\right)\right|^{2} / \Delta\right)$ dependence associated with the index of refraction for the classical field. For the Faraday interaction the same factor appears in the propagation of the quantum field and both factors can be omitted.

\section{Inclusion of spontaneous emission}

In Sec. II.B we derive equations of motion ignoring spontaneous emission. To include spontaneous emission we should modify Eq. 92 such that it contains a decay as well as the Langevin noise operators associated with the decay. The general treatment of the Langevin noise operators is quite complicated because they depend on the particular decay mechanism. In particular whether the atoms end up in the states $|0\rangle$ and $|1\rangle$ or some auxiliary states $\left|a_{m}\right\rangle$ (Fig. 2) after a decay will influence the equations for the parametric gain and Faraday interaction. For the beam splitter interaction on the other hand the precise state that the atoms decay to is less important (Gorshkov et al., 2007b c). Here we will for simplicity only consider the decay to some auxiliary states $\left|a_{m}\right\rangle$.

To describe spontaneous emission we assume that each of the excited states $\left|e_{m}\right\rangle_{j}$ of the $j$ th atom couples to some state $\left|a_{m}\right\rangle_{j}$ via a continuum of modes described by the annihilation operators $b_{j}(\omega)$. We assume that each atom couples to its own continuum. By doing so we ignore collective scattering effects such as superradiance or Bragg scattering. The decay of the $j$ th atom may then be described as

$$
\begin{aligned}
H_{\text {decay }}^{j}= & \int d \omega\left[b_{j}^{\dagger}(\omega) b_{j}(\omega)\right. \\
& \left.+\rho(\omega) \sum_{m}\left(g_{m}(\omega)\left|e_{m}\right\rangle_{j}\left\langle a_{m}\right| b_{j}(\omega)+\text { H.C. }\right)\right],
\end{aligned}
$$

where $g_{m}(\omega)$ is the coupling constant of the $m$ th excited state, and $\rho(\omega)$ is the density of states of the continuum. To arrive at the equations of motion for the atomic operators we first derive the equations of motion for $b_{j}(\omega)$ using the Hamiltonian 100 . We then formally solve this equation by integrating over time and substitute the result into the equation for $\left|g_{m}\right\rangle_{j}\left\langle e_{m}\right|$. In the Markov approximation (Barnett and Radmore, 1997) Eq. 92 acquires the additional terms

$$
\frac{d}{d t}\left|g_{m}\right\rangle_{j}\left\langle e_{m^{\prime}}\left|=\ldots-\frac{\gamma_{m^{\prime}}}{2}\right| g_{m}\right\rangle_{j}\left\langle e_{m^{\prime}}\right|+\sqrt{\gamma_{m}} F_{g m, e m^{\prime}}^{j}(t),
$$

where we have ignored the Lamb shift. The decay and noise operators are given by

$$
\gamma_{m}=2 \pi\left|g_{m}\left(\omega_{m}\right)\right|^{2} \rho\left(\omega_{m}\right)
$$

and

$$
\begin{aligned}
F_{g m, e m^{\prime}}^{j}(t)= & \frac{-i\left|g_{m}\right\rangle_{j}\left\langle a_{m^{\prime}}\right|}{\sqrt{\gamma_{m^{\prime}}}} \\
& \times \int d \omega g_{m^{\prime}}(\omega) b_{j}(\omega, t=0) \mathrm{e}^{-i\left(\omega-\omega_{m^{\prime}}\right) t}
\end{aligned}
$$

with $\omega_{m}$ being the transition frequency for the $\left|e_{m}\right\rangle$ to $\left|a_{m}\right\rangle$ transition. Note that if there is a difference between the population decay rate and twice the decay rate of the polarization, e.g., due to collisional broadening, the decay rate $\gamma_{m} / 2$ appearing here should be the polarization decay rate. The correlation functions within the Markov approximation are

$$
\begin{aligned}
\left\langle F_{g m, e m^{\prime}}^{j \dagger} F_{g m^{\prime \prime}, e m^{\prime \prime \prime}}^{j^{\prime}}\right\rangle & \approx 0 \\
\left\langle F_{g m, e m^{\prime}}^{j} F_{g m^{\prime \prime}, e m^{\prime \prime \prime}}^{j^{\prime} \dagger}\right. & \approx \delta\left(t-t^{\prime}\right) \delta_{m^{\prime}, m^{\prime \prime \prime}} \delta_{j, j^{\prime}}\left\langle\mid g_{m}\right\rangle\left\langle g_{m^{\prime \prime}} \mid\right\rangle .
\end{aligned}
$$

Since most of the atoms are always in the state $|0\rangle$ the only important noise operators are $F_{g 0, e m}$.

The equations of motion that we derive in Sec. II.B are due to the time evolution of $a_{L}$ and the time evolution of $j_{+}(\vec{r})$ used in the definition of $a_{A}$ in Eq. (21). Because the decay simply adds a term in Eq. (101) which is similar to the detuning term in Eq. (92) we can obtain the equations of motion for these operators by using the replacement in Eq. 42. Note that for operators $\left|g_{m}\right\rangle\left\langle e_{m^{\prime}}\right|$ the detuning and decay appears in the combination $\Delta-i \gamma / 2$ so that, e.g., for the ground state operators $\left|g_{m}\right\rangle\left\langle g_{m^{\prime}}\right|$ coupling to $\left|g_{m}\right\rangle\left\langle e_{m^{\prime \prime}}\right|$ one should use the minus sign in the substitution. On the other hand if the coupling is to operators $\left|e_{m^{\prime \prime}}\right\rangle\left\langle g_{m^{\prime}}\right|$ one should use the plus sign. In order to conserve the commutation relation for the operators $a_{A}$ and $a_{L}$ one should also include the Langevin noise operators and the time derivative of $\left\langle j_{x}(\vec{r})\right\rangle$ used in the definition of $a_{A}(21)$.

For the beam splitter interaction the time derivative of $\left\langle j_{x}(\vec{r})\right\rangle$ can be ignored because the strong classical field couples to an almost empty transition. The equation of motion can therefore be obtained simply by making the substitution (42). The equation of motion for the light (35) arises from the commutator of $a_{L}$ with the Hamiltonian (90). In the resulting equation of motion $a_{L}$ couples to $|0\rangle\langle e|$ and we should therefore use the minus sign in Eq. 42 . The atomic annihilation operator is proportional to $|0\rangle\langle 1|$, which couples to $|0\rangle\langle e|$ and $|e\rangle\langle 1|$. The contribution from $|e\rangle\langle 1|$, is however very small and has been neglected in Eq. (35), and we should once again use the minus sign in the substitution (42). Ignoring the noise operators we arrive at the equations of motion (43). Including the noise operators gives the additional terms

$$
\begin{aligned}
\frac{\partial}{\partial z} a_{L}(z, t) & =\ldots+\frac{\sqrt{\gamma} g^{*}}{\Delta-i \frac{\gamma}{2}} F(z, t) \\
\frac{\partial}{\partial t} a_{A}(z, t) & =\ldots-\frac{\sqrt{\gamma} \Omega^{*}}{2 \Delta-i \gamma} F(z, t) .
\end{aligned}
$$


The noise $F(z, t)$ appearing here is defined by first defining

$$
F(\vec{r}, t)=\frac{1}{\sqrt{n(\vec{r}, t)}} \sum_{j} F_{g 0, e}^{j}(t) \delta\left(\vec{r}-\vec{r}_{j}\right)
$$

Integrating the equations of motion over the transverse coordinates we define $F(z, t)$ in analogy with Eq. (29) (ignoring the mode index $m$ for a single mode). The resulting operator has the standard expectation value of vacuum noise $\left\langle F^{\dagger}(z, t) F\left(z^{\prime}, t^{\prime}\right)\right\rangle=0,\left\langle F(z, t) F^{\dagger}\left(z^{\prime}, t^{\prime}\right)\right\rangle=$ $\delta\left(z-z^{\prime}\right) \delta\left(t-t^{\prime}\right)$. The addition of these noise operators ensures that the atom and light operators retains the correct commutation relations.

For the parametric gain the situation is a little different. The light operator $a_{L}$ couples to the coherence $|1\rangle\langle e|$ and we should still use the substitution $(42)$ with the minus sign. The atomic coherence $|0\rangle\langle 1|$ again couples to $|0\rangle\langle e|$ and $|e\rangle\langle 1|$, but now we can no longer neglect $|e\rangle\langle 1|$, which gives rise to the AC-Stark shift [the first term in the spin equation in Eq. [360]. We should therefore use the plus sign in the substitution (42) for this term.

To include the change of the mean spin $\left\langle j_{x}(\vec{r}, t)\right\rangle$ we include the decay of the densities $n_{0}$ and $n_{1}$ of atoms in states $|0\rangle$ and $|1\rangle$ given by

$$
\begin{aligned}
& \frac{d}{d t} n_{0}(\vec{r}, t) \approx-\frac{\gamma|\Omega(\vec{r}, t)|^{2}}{4 \Delta^{2}+\gamma^{2}} n_{0}(\vec{r}, t) . \\
& \frac{d}{d t} n_{1}(\vec{r}, t) \approx 0
\end{aligned}
$$

Since we also assume that the decay takes the atoms to some auxiliary state $\left|a_{m}\right\rangle$, an initially fully polarized state will remain fully polarized so that $\left\langle j_{x}(\vec{r}, t)\right\rangle \approx$ $n_{0}(\vec{r}, t) / 2$ as long as the interaction with the quantum field is weak (note, however, that we are describing a situation leading to superradiant scattering so that this approximation may break down very quickly). The contribution from the time derivative of the mean spin then exactly cancels the decay of $a_{A}(z, t)$ arising from the substitution (42). Since there is no longer any decay, one also finds that the resulting equations of motion (53) do not contain any noise.

Finally for the Faraday interaction the easiest way to proceed is again to combine the results for the beam splitter interaction and the parametric gain. Similar to the discussion in Appendix IX.B the equations of motion for the Faraday interaction can therefore be obtained by subtracting the right hand side of Eq. 53 from the right hand side of Eq. 43 and dividing by $\sqrt{2}$. Furthermore, we again ignore the change of the propagation caused by the index of refraction because this is accompanied by a similar change in the propagation of the classical light. The resulting equations in terms of the $x$ and $p$ operators are given in Eq. (54) without the noise operators. The noise operators give the additional terms

$$
\begin{aligned}
& \frac{\partial}{\partial z} x_{L}(z, t)=\ldots+\frac{g \sqrt{2 \gamma}}{\sqrt{4 \Delta^{2}+\gamma^{2}}} F_{x}(z, t) \\
& \frac{\partial}{\partial z} p_{L}(z, t)=\ldots+\frac{g \sqrt{2 \gamma}}{\sqrt{4 \Delta^{2}+\gamma^{2}}} F_{p}(z, t) \\
& \frac{\partial}{\partial t} x_{A}(z, t)=\ldots-\frac{\Omega \sqrt{\gamma}}{\sqrt{4 \Delta^{2}+\gamma^{2}}} F_{x}(z, t) \\
& \frac{\partial}{\partial t} p_{A}(z, t)=\ldots-\frac{\Omega \sqrt{\gamma}}{\sqrt{4 \Delta^{2}+\gamma^{2}}} F_{p}(z, t) .
\end{aligned}
$$

Here we have for simplicity assumed $\Omega$ and $g$ to be real and have defined new noise operators

$$
\begin{aligned}
& F_{x}(z, t)=\frac{1}{\sqrt{2}}\left(\mathrm{e}^{i \phi} F(z, t)+\mathrm{e}^{-i \phi} F^{\dagger}(z, t)\right) \\
& F_{p}(z, t)=\frac{1}{\sqrt{2} i}\left(\mathrm{e}^{i \phi} F(z, t)-\mathrm{e}^{-i \phi} F^{\dagger}(z, t)\right),
\end{aligned}
$$

which have the standard commutation relation $\left[F_{x}(z, t), F_{p}\left(z^{\prime}, t^{\prime}\right)\right]=i \delta\left(z-z^{\prime}\right) \delta\left(t-t^{\prime}\right)$.

\section{E. Dimensionless equations of motion}

Casting the equations of motion for all three basic interactions in a dimensionless form allows to see that the coupling constant $\kappa$ for the Faraday interaction (56), naturally plays the role of the coupling constant for all protocols. We introduce the dimensionless position and time coordinates $s=z / L$ and $v=h(0, t) / h(0, T)$ running from 0 to 1 . The rescaled time coordinate simplifies the equations because it is proportional to the total integrated intensity of the field. In the weak saturation limit the dynamics is completely controlled by the incident number of photons in the classical field. Changing the intensity will thus influence the temporal dynamics of the system, but the final state primarily depends on the total number of incident photons. When using such rescaled coordinates it is desirable to also change the field operators such that, e.g., an incident light field operator which is normalized in time $\left[a_{L}(t), a_{L}^{\dagger}\left(t^{\prime}\right)\right]=\delta\left(t-t^{\prime}\right)$ is now normalized relative to the new time variable $\left[a_{L}(v), a_{L}^{\dagger}\left(v^{\prime}\right)\right]=\delta\left(v-v^{\prime}\right)$. This normalization is achieved with the rescaling

$$
\begin{aligned}
\tilde{a}_{A}(s=z / L) & =\sqrt{L} a_{A}(z) \\
\tilde{a}_{L}(v(t)) & =-\frac{\kappa \sqrt{4 \Delta^{2}+\gamma^{2}}}{\sqrt{d \gamma} \Omega(t)} a_{L}(t),
\end{aligned}
$$

where we have used the dimensionless coupling constant $\kappa=\sqrt{h(0, T)}$, which appeared in the solution for the Faraday interaction, c.f., Eq. (57) in the far off-resonant limit $\Delta \gg \gamma$. Below we omit the tilde on the operators on the left hand side. In these new rescaled variables the equations of motion for the beam splitter interaction 
become

$$
\begin{aligned}
\frac{\partial}{\partial s} a_{L}(s, v) & =i \frac{\gamma d}{2(2 \Delta-i \gamma)} a_{L}(s, v)-i \kappa \frac{e^{i \phi}}{2} a_{A}(s, v) \\
\frac{\partial}{\partial v} a_{A}(s, v) & =i \kappa^{2} \frac{\Delta+i \frac{\gamma}{2}}{\gamma d} a_{A}(s, v)-i \kappa \frac{e^{i \phi}}{2} a_{L}(s, v) .
\end{aligned}
$$

With the same rescaling for the parametric gain interaction we find

$$
\begin{aligned}
& \frac{\partial}{\partial s} a_{L}(s, v)=-i \kappa \frac{e^{i \phi}}{2} a_{A}^{\dagger}(s, v) \\
& \frac{\partial}{\partial v} a_{A}(s, v)=-i \frac{\kappa^{2} \Delta}{\gamma d} a_{A}(s, v)-i \kappa \frac{e^{i \phi}}{2} a_{L}^{\dagger}(s, v)
\end{aligned}
$$

where we have again assumed that $g$ is real.

For the Faraday interaction the equations of motion become

$$
\begin{aligned}
\frac{\partial}{\partial s} x_{L}(s, v) & =\kappa p_{A}(s, v)-\frac{\gamma^{2}}{4 \Delta^{2}+\gamma^{2}} \frac{d}{2} x_{L}(s, v) \\
\frac{\partial}{\partial s} p_{L}(s, v) & =-\frac{\gamma^{2}}{4 \Delta^{2}+\gamma^{2}} \frac{d}{2} p_{L}(s, v) \\
\frac{\partial}{\partial v} x_{A}(s, v) & =\kappa p_{L}(s, v)-\frac{\kappa^{2}}{2 d} x_{A}(s, v) \\
\frac{\partial}{\partial v} p_{A}(s, v) & =-\frac{\kappa^{2}}{2 d} p_{A}(s, v)
\end{aligned}
$$

where we have ignored small corrections which vanish in the far detuned limit. From the equations derived here we see that $\kappa$ naturally plays the role of the coupling constant for all the protocols.

\section{F. Tensor Decomposition}

The coefficients determining the strength of the irreducible tensor components are

$$
\begin{aligned}
& a_{k}(\Delta)=(-)^{1+F} c_{k}(2 k+1) \sqrt{\frac{2 F+1}{3}} \\
& \times\left[\sum_{F^{\prime}} \frac{(-)^{F^{\prime}}}{1-\delta_{F^{\prime}} / \Delta}\left(2 F^{\prime}+1\right)\left\{\begin{array}{ccc}
J^{\prime} & F^{\prime} & I \\
F & J & 1
\end{array}\right\}^{2}\left\{\begin{array}{ccc}
F & k & F \\
1 & F^{\prime} & 1
\end{array}\right\}\right],
\end{aligned}
$$

where the expressions in curly brackets are $6 j$-symbols, $\Delta=\Delta_{F+1}, \delta_{F^{\prime}}=\Delta_{F+1}-\Delta_{F^{\prime}}$ and

$$
\begin{aligned}
& c_{0}=1, \quad c_{1}=\sqrt{\frac{2}{F(F+1)}}, \\
& c_{2}=-\frac{3}{\sqrt{10 F(F+1)(2 F-1)(2 F+3)}} .
\end{aligned}
$$

The last line is valid for $F>1 / 2$, that is nuclear spin $I \neq 0$, and has to be replaced by $c_{2}=0$ for $I=0$.
In the asymptotic limit of large (blue) detuning, $-\Delta \gg$ $\delta_{F^{\prime}}$, the sum in square brackets can be simplified by means of

$$
\begin{aligned}
& \sum_{F^{\prime}=F-1}^{F+1}(-)^{F^{\prime}}\left(2 F^{\prime}+1\right)\left\{\begin{array}{ccc}
J^{\prime} & F^{\prime} & I \\
F & J^{\prime} & 1
\end{array}\right\}^{2}\left\{\begin{array}{ccc}
F & k & F \\
1 & F^{\prime} & 1
\end{array}\right\}= \\
& (-)^{\left(2 J+2 F+J^{\prime}+I+k\right)}\left\{\begin{array}{ccc}
J & I & F \\
F & k & J
\end{array}\right\}\left\{\begin{array}{lll}
J & J & k \\
1 & 1 & J^{\prime}
\end{array}\right\}
\end{aligned}
$$

to get

$$
\begin{aligned}
a_{k}=\lim _{\Delta \rightarrow-\infty} a_{k}(\Delta)= & (-)^{2 J+F+J^{\prime}+I+k+1} c_{k}(2 k+1) \\
& \times \sqrt{\frac{2 F+1}{3}}\left\{\begin{array}{ccc}
J & I & F \\
F & k & J
\end{array}\right\}\left\{\begin{array}{lll}
J & J & k \\
1 & 1 & J^{\prime}
\end{array}\right\} .
\end{aligned}
$$

From this expression it is evident that $a_{2}$ has to vanish because the triple $\{J, J, k\}=\{1 / 2,1 / 2,2\}$ does not satisfy the triangle inequality. For the particular case of the Cesium $(I=7 / 2) D_{2}$-line at $F=4 \rightarrow F^{\prime}=3,4,5$ the asymptotic values of the non-vanishing coefficients are

$$
a_{0}=1 / 6, \quad a_{1}=1 / 24 .
$$

\section{References}

Achilles, D., C. Silberhorn, C. Sliwa, K. Banaszek, I. A. Walmsley, M. J. Fitch, B. C. Jacobs, T. B. Pittman, and J. D. Franson, 2004, Journal Of Modern Optics 51, 1499, http://dx.doi.org/10.1080/09500340408235288

Adesso, G., and F. Illuminati, 2007, J. Phys. A 40, 7821, http://dx.doi.org/10.1088/1751-8113/40/28/S01

Alexander, A. L., J. J. Longdell, M. J. Sellars, and N. B. Manson, 2006, Phys. Rev. Lett. 96, 043602, http://link. aps.org/abstract/PRL/v96/e043602

Alexandrov, E., 2003, Physica Scripta T105, 27, http://dx. doi.org/10.1238/Physica.Topical.105a00027

André, A., 2005, Nonclassical States of Light and Atomic Ensembles: Generation and New Applications, Ph.D. thesis, Harvard University.

André, A., M. Bajcsy, A. S. Zibrov, and M. D. Lukin, 2005, Phys. Rev. Lett. 94, 063902, http://link.aps.org/ abstract/PRL/v94/e063902

Andre, A., L. Duan, and M. Lukin, 2002, Phys. Rev. Lett. 88, http://dx.doi.org/10.1103/PhysRevLett.88.243602

André, A., and M. D. Lukin, 2002, Phys. Rev. Lett. 89, 143602, http://dx.doi.org/10.1103/PhysRevLett. 89.143602

Appel, J., E. Figueroa, D. Korystov, M. Lobino, and A. I. Lvovsky, 2008, Physical Review Letters 100, 093602, http: //link.aps.org/abstract/PRL/v100/e093602

Arecchi, F. T., E. Courtens, R. Gilmore, and H. Thomas, 1972, Phys. Rev. A 6, 2211, http://dx.doi.org/10.1103/ PhysRevA.6.2211

Aspelmeyer, M., T. Jennewein, M. Pfennigbauer, W. R. Leeb, and A. Zeilinger, 2003, Ieee Journal Of Selected Topics In Quantum Electronics 9, 1541, http://dx.doi.org/10. 1109/JSTQE. 2003.820918

Atature, M., J. Dreiser, A. Badolato, and A. Imamoglu, 2007, Nat Phys 3, 101, http://dx.doi.org/10.1038/nphys521

Bajcsy, M., A. S. Zibrov, and M. D. Lukin, 2003, Nature 426, 638, http://dx.doi.org/10.1038/nature02176. 
Balić, V., D. A. Braje, P. Kolchin, G. Y. Yin, and S. E. Harris, 2005, Phys. Rev. Lett. 94, 183601, http://link.aps.org/ abstract/PRL/v94/e183601

Barnett, S. M., and P. M. Radmore, 1997, Methods in Theoretical Quantum Optics (Oxford University Press, Oxford).

Barrett, M., J. Chiaverini, T. Schaetz, J. Britton, W. Itano, J. Jost, E. Knill, C. Langer, D. Leibfried, R. Ozeri, and D. Wineland, 2004, Nature 429, 737, http://dx.doi.org/ $10.1038 /$ nature02608

Bartlett, S. D., B. C. Sanders, S. L. Braunstein, and K. Nemoto, 2002, Phys. Rev. Lett. 88, 097904, http: //dx.doi.org/10.1103/PhysRevLett.88.097904

Bennett, C., G. Brassard, C. Crpeau, R. Jozsa, A. Peres, and W. Wootters, 1993, Phys. Rev. Lett. 70, 1895, http: //link.aps.org/abstract/PRL/v70/p1895

Black, A. T., J. K. Thompson, and V. Vuletić, 2005, Phys. Rev. Lett. 95, 133601, http://link.aps.org/abstract/ PRL/v95/e133601.

Boller, K.-J., A. Imamolu, and S. E. Harris, 1991, Phys. Rev. Lett. 66, 2593, http://dx.doi.org/10.1103/ PhysRevLett.66.2593

Bouchoule, I., and K. Mølmer, 2002, Phys. Rev. A 66, 043811, http://dx.doi.org/10.1103/PhysRevA.66.043811.

Bouwmeester, D., J. Pan, K. Mattle, M. Eibl, H. Weinfurter, and A. Zeilinger, 1997, Nature 390, 575, http://dx.doi. org/10.1038/37539

Bowen, W. P., N. Treps, B. C. Buchler, R. Schnabel, T. C. Ralph, H.-A. Bachor, T. Symul, and P. K. Lam, 2003a, Phys. Rev. A 67, 032302, http://dx.doi.org/10.1103/ PhysRevA.67.032302.

Bowen, W. P., N. Treps, B. C. Buchler, R. Schnabel, T. C. Ralph, T. Symul, and P. K. Lam, 2003b, IEEE J. Sel. Top. Quant.El. 9, 1519, http://arxiv.org/abs/ quant-ph/0303179

Braginsky, V. B., and F. Y. Khalili, 1996, Rev. Mod. Phys. 68, 1, http://dx.doi.org/10.1103/RevModPhys.68.1.

Braje, D. A., V. Balić, S. Goda, G. Y. Yin, and S. E. Harris, 2004, Phys. Rev. Lett. 93, 183601, http://dx.doi.org/ 10.1103/PhysRevLett.93.183601

Brask, J. B., and A. S. Sørensen, 2008, eprint arXiv:0803.2069v2, http://arxiv.org/abs/0803.2069

Braunstein, S., and H. Kimble, 1998, Phys. Rev. Lett. 80, 869, http://link.aps.org/abstract/PRL/v80/p869.

Braunstein, S. L., 2005, Phys. Rev. A 71, 055801, http:// link.aps.org/abstract/PRA/v71/e055801

Braunstein, S. L., C. A. Fuchs, and H. J. Kimble, 2000, Journal Of Modern Optics 47, 267, http://arxiv.org/abs/ quant-ph/9910030

Braunstein, S. L., C. A. Fuchs, H. J. Kimble, and P. van Loock, 2001, Phys. Rev. A 64, 022321, http://link.aps. org/abstract/PRA/v64/e022321.

Braunstein, S. L., and P. van Loock, 2005, Rev. Mod. Phys. 77, 513, http://link.aps.org/abstract/RMP/v77/p513.

Briegel, H.-J., W. Dür, J. I. Cirac, and P. Zoller, 1998, Phys. Rev. Lett. 81, 5932, http://dx.doi.org/10.1103/ PhysRevLett.81.5932

Brion, E., K. Mølmer, and M. Saffman, 2007, Phys. Rev. Lett. 99, 260501, http://link.aps.org/abstract/PRL/ v99/e260501.

Camacho, R. M., C. J. Broadbent, I. Ali-Khan, and J. C. Howell, 2007, Phys. Rev. Lett. 98, 043902, http://link. aps.org/abstract/PRL/v98/e043902.

Carman, R. L., F. Shimizu, C. S. Wang, and N. Bloembergen, 1970, Phys. Rev. A 2, 60, http://dx.doi.org/10.1103/
PhysRevA.2.60.

Cerf, N. J., O. Krüger, P. Navez, R. F. Werner, and M. M. Wolf, 2005, Phys. Rev. Lett. 95, 070501, http://link. aps.org/abstract/PRL/v95/e070501

Chaikin, P. M., and T. C. Lubensky, 1995, Principles of condensed matter physics (Cambridge University Press, Cambridge, United Kingdom).

Chaneliere, T., D. Matsukevich, S. Jenkins, S. L. T.A.B., Kennedy, and A. Kuzmich, 2005, Nature 438, 833, http: //dx.doi.org/10.1038/nature04315

Chaneliere, T., D. N. Matsukevich, S. D. Jenkins, S.-Y. Lan, R. Zhao, T. A. B. Kennedy, and A. Kuzmich, 2007, Phys. Rev. Lett. 98, 113602, http://link.aps.org/abstract/ PRL/v98/e113602

Chaudhury, S., G. A. Smith, K. Schulz, and P. S. Jessen, 2006, Phys. Rev. Lett. 96, 043001, http://link.aps.org/ abstract/PRL/v96/e043001.

Chen, Y.-A., S. Chen, Z.-S. Yuan, B. Zhao, C.-S. Chuu, J. Schmiedmayer, and J.-W. Pan, 2008, Nat. Phys. 4, 103, http://dx.doi.org/10.1038/nphys832

Chen, Z.-B., B. Zhao, Y.-A. Chen, J. Schmiedmayer, and J.W. Pan, 2007, Phys. Rev. A 76, 022329, http://link. aps.org/abstract/PRA/v76/e022329

Childress, L., J. M. Taylor, A. S. Sørensen, and M. D. Lukin, 2005, Phys. Rev. A 72, 052330, http://link.aps.org/ abstract/PRA/v72/e052330.

Choi, K. S., H. Deng, J. Laurat, and H. J. Kimble, 2008, Nature 452, 67, http://dx.doi.org/10.1038/nature06670.

Chou, C., S. Polyakov, A. Kuzmich, and H. Kimble, 2004, Phys Rev. Lett. 92, 213601, http://link.aps.org/ abstract/PRL/v92/e213601.

Chou, C.-W., J. Laurat, H. Deng, K. S. Choi, H. de Riedmatten, D. Felinto, and H. J. Kimble, 2007, Science 316, 1316, http://www.sciencemag.org/cgi/content/ abstract/316/5829/1316

Chou, C. W., H. de Riedmatten, D. Felinto, S. V. Polyakov, S. J. van Enk, and H. J. Kimble, 2005, Nature 438, 828, http://dx.doi.org/10.1038/nature04353.

Cola, M., M. Paris, N. Piovella, and R. Bonifacio, 2004, J. Phys. B 37, S187, http://dx.doi.org/10.1088/ 0953-4075/37/7/064

Dantan, A., A. Bramati, and M. Pinard, 2005, Phys. Rev. A 71, 043801, http://link.aps.org/abstract/PRA/v71/ e043801.

Dantan, A., J. Cviklinski, E. Giacobino, and M. Pinard, 2006a, Physical Review Letters 97, 023605, http://link. aps.org/abstract/PRL/v97/e023605

Dantan, A., J. Cviklinski, M. Pinard, and P. Grangier, 2006b, Phys. Rev. A 73, 032338, http://link.aps.org/ abstract/PRA/v73/e032338.

Dantan, A., and M. Pinard, 2004, Phys. Rev. A 69, 043810, http://dx.doi.org/10.1103/PhysRevA.69.043810

Deutsch, I. H., and P. S. Jessen, 1998, Phys. Rev. A 57, 1972, http://dx.doi.org/10.1103/PhysRevA.57.1972

Dorner, U., A. Klein, and D. Jaksch, 2008, Quant. Inf. Comp. 8, 0468, http://arxiv.org/abs/0711.1831.

Du, S., P. Kolchin, C. Belthangady, G. Y. Yin, and S. E. Harris, 2008, Physical Review Letters 100, 183603, http: //link.aps.org/abstract/PRL/v100/e183603.

Duan, L. M., J. I. Cirac, and P. Zoller, 2002, Phys. Rev. A 66, 023818, http://dx.doi.org/10.1103/PhysRevA.66. 023818

Duan, L.-M., J. I. Cirac, P. Zoller, and E. S. Polzik, 2000a, Phys. Rev. Lett. 85, 5643, http://dx.doi.org/10.1103/ 
PhysRevLett.85.5643

Duan, L.-M., G. Giedke, J. I. Cirac, and P. Zoller, 2000b, Phys. Rev. Lett. 84, 2722, http://dx.doi.org/10.1103/ PhysRevLett.84.2722

Duan, L.-M., M. D. Lukin, J. I. Cirac, and P. Zoller, 2001, Nature 414, 413, http://dx.doi.org/10.1038/35106500

Dür, W., and H. J. Briegel, 2007, Rep. Prog. Phys. 70, 1381, http://stacks.iop.org/0034-4885/70/1381.

Dür, W., H.-J. Briegel, J. I. Cirac, and P. Zoller, 1999, Phys. Rev. A 59, 169, http://dx.doi.org/10.1103/PhysRevA. 59.169 .

de Echaniz, S. R., M. Koschorreck, M. Napolitano, M. Kubasik, and M. W. Mitchell, 2008, Phys. Rev. A 77, http://dx.doi.org/10.1103/PhysRevA.77.032316.

Eckert, K., O. Romero-Isart, M. Rodriguez, M. Lewenstein, E. S. Polzik, and A. Sanpera, 2008, Nat Phys 4, 50, http: //dx.doi.org/10.1038/nphys776.

Edmonds, A., 1964, Drehimpulse in der Quantenmechanik (Bibliographisches Institut, Mannheim).

Einstein, A., B. Podolsky, and N. Rosen, 1935, Phys. Rev. 47, 777, http://dx.doi.org/10.1103/PhysRev.47.777.

Eisaman, M., A. Andre, F. Massou, M. Fleischhauer, A. Zibrov, and M. Lukin, 2005, Nature 438, 837, http://dx. doi.org/10.1038/nature04327

Eisert, J., and M. B. Plenio, 2003, Int. J. Quant. Inf. 1, 479, http://arxiv.org/abs/quant-ph/0312071.

Eisert, J., S. Scheel, and M. B. Plenio, 2002, Phys. Rev. Lett. 89, 137903, http://dx.doi.org/10.1103/PhysRevLett. 89.137903.

van Enk, S. J., N. Lütkenhaus, and H. J. Kimble, 2007, Phys. Rev. A 75, 052318, http://link.aps.org/abstract/PRA/ v75/e052318.

Fiurăśek, J., 2002, Phys. Rev. Lett. 89, 137904, http:// arxiv.org/abs/quant-ph/0204069.

Fiurăśek, J., 2003, Phys. Rev. A 68, 022304, http://link. aps.org/abstract/PRA/v68/e022304.

Fiurăśek, J., H. J. Cerf, and E. S. Polzik, 2004, Phys. Rev. Lett. 93, 180501, http://link.aps.org/abstract/PRL/ v93/e180501.

Fiurăśek, J., J. Sherson, T. Opatrny, and E. S. Polzik, 2006, Phys. Rev. A 73, 022331, http://arxiv.org/abs/ quant-ph/0510099

Fleischhauer, M., A. Imamoglu, and J. P. Marangos, 2005, Rev. Mod. Phys. 77, 633, http://link.aps.org/ abstract/RMP/v77/p633

Fleischhauer, M., and M. D. Lukin, 2000, Phys. Rev. Lett. 84, 5094, http://dx.doi.org/10.1103/PhysRevLett.84. 5094

Fleischhauer, M., and T. Richter, 1995, Phys. Rev. A 51, 2430, http://dx.doi.org/10.1103/PhysRevA.51.2430.

Fraval, E., M. J. Sellars, and J. J. Longdell, 2005, Phys. Rev. Lett. 95, 030506, http://link.aps.org/abstract/ PRL/v95/e030506.

Furusawa, A., J. Sorensen, S. Braunstein, C. Fuchs, H. Kimble, and E. Polzik, 1998, Science 282, 706, http://dx.doi. org/10.1126/science.282.5389.706.

Furusawa, A., and N. Takei, 2007, Phys. Rep. 443, 97, http:

//dx.doi.org/10.1016/j.physrep.2007.03.001

Genes, C., and P. R. Berman, 2006, Phys. Rev. A 73, 013801, http://link.aps.org/abstract/PRA/v73/e013801.

Geremia, J. M., J. K. Stockton, and H. Mabuchi, 2004, Science 304, 270, http://dx.doi.org/10.1126/science. 1095374.

Geremia, J. M., J. K. Stockton, and H. Mabuchi, 2006, Phys.
Rev. A 73, 042112, http://link.aps.org/abstract/PRA/ v73/e042112.

Giedke, G., and J. I. Cirac, 2002, Phys. Rev. A 66, 032316, http://dx.doi.org/10.1103/PhysRevA.66.032316

Giedke, G., M. M. Wolf, O. Krüger, R. F. Werner, and J. I. Cirac, 2003, Phys. Rev. Lett. 91, 107901, http://dx.doi. org/10.1103/PhysRevLett.91.107901.

Gorshkov, A. V., A. André, M. Fleischhauer, A. S. Sørensen, and M. D. Lukin, 2007a, Phys. Rev. Lett. 98, 123601, http://link.aps.org/abstract/PRL/v98/e123601.

Gorshkov, A. V., A. André, M. D. Lukin, and A. S. Sørensen, 2007b, Phys. Rev. A 76, 033804, http://link.aps.org/ abstract/PRA/v76/e033804.

Gorshkov, A. V., A. André, M. D. Lukin, and A. S. Sørensen, 2007c, Phys. Rev. A 76, 033805, http://link.aps.org/ abstract/PRA/v76/e033805

Gorshkov, A. V., A. André, M. D. Lukin, and A. S. Sørensen, 2007d, Phys. Rev. A 76, 033806, http://link.aps.org/ abstract/PRA/v76/e033806

Gorshkov, A. V., T. Calarco, M. D. Lukin, and A. S. Sørensen, 2008, Phys. Rev. A 77, 043806, http://arxiv.org/abs/ 0710.2698

Groeger, S., G. Bison, J. Schenker, R. Wynands, and A. Weis, 2006, European Physical Journal D 38, 239, http://dx. doi.org/10.1140/epjd/e2006-00037-y

Grosshans, F., and P. Grangier, 2001, Phys. Rev. A 64, 010301, see also quant-ph/0009079v1 and quantph/0010107v2, http://link.aps.org/abstract/PRA/ v64/e010301.

Hald, J., J. L. Sørensen, C. Schori, and E. S. Polzik, 1999, Phys. Rev. Lett. 83, 1319, http://link.aps.org/ abstract/PRL/v83/p1319

Hammerer, K., 2006, Quantum Information Processing with Atomic Ensembles and Light, Ph.D. thesis, Technical University Munich.

Hammerer, K., M. Aspelmeyer, E. S. Polzik, and P. Zoller., 2008, arXiv:0804.3005 http://arxiv.org/abs/0804.3005.

Hammerer, K., K. Mølmer, E. Polzik, and J. Cirac, 2004, Phys. Rev. A. 70, 044304, http://link.aps.org/ abstract/PRA/v70/e044304.

Hammerer, K., E. S. Polzik, and J. I. Cirac, 2005a, Phys. Rev. A 72, 052313, http://link.aps.org/abstract/PRA/v72/ e052313.

Hammerer, K., E. S. Polzik, and J. I. Cirac, 2006, Phys. Rev. A 74, 064301, http://link.aps.org/abstract/PRA/v74/ e064301.

Hammerer, K., M. M. Wolf, E. S. Polzik, and J. I. Cirac, 2005b, Phys. Rev. Lett. 94, 150503, http://dx.doi.org/ 10.1103/PhysRevLett.94.150503

Hansel, W., P. Hommelhoff, T. W. Hansch, and J. Reichel, 2001, Nature 413, 498, http://dx.doi.org/10. 1038/35097032.

Happer, W., 1972, Rev. Mod. Phys. 44, 169, http://link. aps.org/abstract/RMP/v44/p169

Happer, W., and B. S. Mathur, 1967, Phys. Rev. 163, 12, http://dx.doi.org/10.1103/PhysRev.163.12.

Hartmann, L., B. Kraus, H.-J. Briegel, and W. Dür, 2007, Phys. Rev. A 75, 032310, http://link.aps.org/ abstract/PRA/v75/e032310.

Hau, L. V., S. E. Harris, Z. Dutton, and C. H. Behroozi, 1999, Nature 397, 594, http://dx.doi.org/10.1038/17561.

Hemmer, P. R., A. V. Turukhin, M. S. Shahriar, and J. A. Musser, 2001, Opt. Lett. 26, 361, http://ol.osa.org/ abstract. cfm?URI=0l-26-6-361. 
Herskind, P., A. Dantan, M. B. Langkilde-Lauesen, A. Mortensen, J. L. Sørensen, and M. Drewsen, 2008, arXiv:0804.4589v1 http://arxiv.org/abs/0804.4589.

Hétet, G., J. J. Longdell, A. L. Alexander, P. K. Lam, and M. J. Sellars, 2008a, Phys. Rev. Lett. 100, 023601, http: //link.aps.org/abstract/PRL/v100/e023601.

Hétet, G., A. Peng, M. T. Johnsson, J. J. Hope, and P. K. Lam, 2008b, Phys. Rev. A 77, 012323, http://link.aps. org/abstract/PRA/v77/e012323

Holland, M. J., M. J. Collett, D. F. Walls, and M. D. Levenson, 1990, Phys. Rev. A 42, 2995, http://link.aps.org/ abstract/PRA/v42/p2995

Holstein, T., and H. Primakoff, 1940, Phys. Rev. 58, 1098, http://link.aps.org/abstract/PR/v58/p1098.

Honda, K., D. Akamatsu, M. Arikawa, Y. Yokoi, K. Akiba, S. Nagatsuka, T. Tanimura, A. Furusawa, and M. Kozuma, 2008, Physical Review Letters 100, 093601, http://link. aps.org/abstract/PRL/v100/e093601

Horoshko, D. B., and S. Y. Kilin, 2000, Phys. Rev. A 61, 032304, http://dx.doi.org/10.1103/PhysRevA.61. 032304

Jackson, J. D., 1975, Classical Electrodynamics (Wiley, New York), 2nd edition.

Jiang, L., J. M. Taylor, and M. D. Lukin, 2007, Phys. Rev. A 76, 012301, http://link.aps.org/abstract/PRA/v76/ e012301.

Josse, V., A. Dantan, A. Bramati, M. Pinard, and E. Giacobino, 2004, Phys. Rev. Lett. 92, 123601, http://dx. doi.org/10.1103/PhysRevLett.92.123601.

Julsgaard, B., 2003, Entanglement and Quantum Interactions with Macroscopic Gas Samples, Ph.D. thesis, University of Aarhus.

Julsgaard, B., A. Kozhekin, and E. S. Polzik, 2001, Nature 413, 400, http://dx.doi.org/doi:10.1038/35096524.

Julsgaard, B., J. Sherson, J. Fiurăśek, J. Cirac, and E. Polzik, 2004a, Nature 432, 482, http://dx.doi.org/doi:10. 1038/nature03064

Julsgaard, B., J. Sherson, J. L. Sorensen, and E. S. Polzik, 2004b, J. of Quantum and Semiclassical Optics B 6, 5, http://dx.doi.org/10.1088/1464-4266/6/1/002

Keyl, M., D. Schlingemann, and R. Werner, 2003, Quantum Information \& Computation 3, 281.

Kimble, H. J., 2008, Nature 453, to appear, http://dx.doi. org/10.1038/nature07127.

Kitagawa, M., and M. Ueda, 1993, Phys. Rev. A 47, 5138, http://link.aps.org/abstract/PRA/v47/p5138

Kittel, C., 1987, Quantum Theory of Solids (Wiley, New York).

Klein, A., U. Dorner, C. M. Alves, and D. Jaksch, 2006, Phys. Rev. A 73, 012332, http://link.aps.org/abstract/PRA/ v73/e012332.

Korbicz, J. K., J. I. Cirac, and M. Lewenstein, 2005a, Phys. Rev. Lett. 95, 120502, http://link.aps.org/abstract/ PRL/v95/e120502.

Korbicz, J. K., J. I. Cirac, and M. Lewenstein, 2005b, Phys. Rev. Lett. 95, 259901, http://link.aps.org/abstract/ PRL/v95/e259901.

Kozhekin, A. E., K. Mølmer, and E. S. Polzik, 2000, Phys. Rev. A. 62, 033809, http://link.aps.org/abstract/PRA/ v62/e033809.

Kraus, B., K. Hammerer, G. Giedke, and J. I. Cirac, 2003, Phys. Rev. A 67, 042314, http://link.aps.org/ abstract/PRA/v67/e042314

Kraus, B., W. Tittel, N. Gisin, M. Nilsson, S. Kröll, and J. I.
Cirac, 2006, Phys. Rev. A 73, 020302(R), http://link. aps.org/abstract/PRA/v73/e020302

Kupriyanov, D. V., O. S. Mishina, I. M. Sokolov, B. Julsgaard, and E. S. Polzik, 2005, Phys. Rev. A 71, 032348, http: //dx.doi.org/10.1103/PhysRevA.71.032348.

Kurnit, N. A., I. D. Abella, and S. R. Hartmann, 1964, Phys. Rev. Lett. 13, 567, http://dx.doi.org/10.1103/ PhysRevLett.13.567

Kurucz, Z., and M. Fleischhauer, 2007, eprint arXiv:0712.2400v1, http://arxiv.org/abs/0712.2400.

Kuzmich, A., N. P. Bigelow, and L. Mandel, 1998, Europhysics Letters 42, 481, http://dx.doi.org/10.1209/ epl/i1998-00277-9.

Kuzmich, A., W. P. Bowen, A. D. Boozer, A. Boca, C. W. Chou, L.-M. Duan, and H. J. Kimble, 2003, Nature 423, 731, http://dx.doi.org/10.1038/nature01714.

Kuzmich, A., and T. A. B. Kennedy, 2004, Phys. Rev. Lett. 92, 030407, http://link.aps.org/abstract/PRL/ v92/e030407.

Kuzmich, A., L. Mandel, and N. P. Bigelow, 2000, Phys. Rev. Lett. 85, 1594, http://dx.doi.org/10.1103/ PhysRevLett.85.1594.

Kuzmich, A., K. Mølmer, and E. S. Polzik, 1997, Phys. Rev. Lett. 79, 4782, http://dx.doi.org/10.1103/ PhysRevLett.79.4782.

Kuzmich, A., and E. Polzik, 2003, Quantum Information with Continuous Variables (Kluwer Academi), chapter Atomic continuous variable processing and light-atoms quantum interface, pp. 231-265.

Kuzmich, A., and E. S. Polzik, 2000, Phys. Rev. Lett. 85, 5639, http://dx.doi.org/10.1103/PhysRevLett.85. 5639

Liu, C., Z. Dutton, C. H. Behroozi, and L. V. Hau, 2001, Nature 409, 490, http://dx.doi.org/10.1038/35054017.

Lloyd, S., and S. L. Braunstein, 1999, Phys. Rev. Lett. 82, 1784, http://dx.doi.org/10.1103/PhysRevLett.82. 1784

Longdell, J. J., E. Fraval, M. J. Sellars, and N. B. Manson, 2005, Phys. Rev. Lett. 95, 063601, http://link.aps.org/ abstract/PRL/v95/e063601

Louchet, A., Y. L. Du, F. Bretenaker, T. Chaneliére, F. Goldfarb, I. Lorgeré, J.-L. L. Gouët, O. Guillot-Noël, and P. Goldner, 2007, arXiv:0710.3231v1 http://arxiv.org/ abs/0710.3231.

Loudon, R., 2004, The Quantum Theory of Light (Oxford University Press).

Lukin, M. D., 2003, Rev. Mod. Phys. 75, 457, http://dx. doi.org/10.1103/RevModPhys.75.457

Lukin, M. D., M. Fleischhauer, R. Cote, L. M. Duan, D. Jaksch, J. I. Cirac, and P. Zoller, 2001, Phys. Rev. Lett. 87, 037901, http://dx.doi.org/10.1103/PhysRevLett. 87.037901

Lukin, M. D., and A. Imamoglu, 2001, Nature 413, 273, http://dx.doi.org/10.1038/35095000

Lukin, M. D., S. F. Yelin, and M. Fleischhauer, 2000, Phys. Rev. Lett. 84, 4232, http://dx.doi.org/10.1103/ PhysRevLett.84.4232.

Mabuchi, H., 2008, private communication.

Madsen, L. B., and K. Mølmer, 2004, Phys. Rev. A 70, 052324, http://dx.doi.org/10.1103/PhysRevA.70. 052324

Mandel, O., M. Greiner, A. Widera, T. Rom, T. W. Hansch, and I. Bloch, 2003, Nature 425, 937, http://dx.doi.org/ 10.1038/nature02008. 
Manz, S., T. Fernholz, J. Schmiedmayer, and J.-W. Pan, 2007, Phys. Rev. A 75, 040101, http://link.aps.org/ abstract/PRA/v75/e040101

Massar, S., and E. S. Polzik, 2003, Phys. Rev. Lett. 91, 060401, http://dx.doi.org/10.1103/PhysRevLett. 91.060401

Massar, S., and S. Popescu, 1995, Phys. Rev. Lett. 74, 1259, http://dx.doi.org/10.1103/PhysRevLett.74.1259.

Matsukevich, D. N., and A. Kuzmich, 2004, Science 306, 663, http://www.sciencemag.org/cgi/content/ abstract/306/5696/663

Menicucci, N. C., P. van Loock, M. Gu, C. Weedbrook, T. C. Ralph, and M. A. Nielsen, 2006, Phys. Rev. Lett. 97, 110501, http://link.aps.org/abstract/PRL/ v97/e110501.

Milonni, P. W., and J. H. Eberly, 1988, Lasers (Wiley, New York).

Mishina, O., D. Kupriyanov, and E. Polzik, 2006, in Proceedings of the NATO Advanced Research Workshop, Crete 2005 Quantum information processing from theory to experiment, volume 199, pp. 346-352.

Mishina, O. S., D. V. Kupriyanov, J. H. Müller, and E. S. Polzik, 2007, Phys. Rev. A 75, 042326, http://dx.doi. org/10.1103/PhysRevA.75.042326

Mišta, L. J., and R. Filip, 2005, Phys. Rev. A 71, 032342, http://link.aps.org/abstract/PRA/v71/e032342.

Moiseev, S., 2003, Opt. Spec. 94, 788.

Moiseev, S. A., and S. Kröll, 2001, Phys. Rev. Lett. 87, 173601, http://dx.doi.org/10.1103/PhysRevLett. 87.173601

Müller, J. H., P. Petrov, D. Oblak, C. L. G. Alzar, S. R. de Echaniz, and E. S. Polzik, 2005, Phys. Rev. A 71, 033803, http://link.aps.org/abstract/PRA/v71/ e033803.

Muschik, C. A., K. Hammerer, E. S. Polzik, and J. I. Cirac, 2006, Phys. Rev. A 73, 062329, http://link.aps.org/ abstract/PRA/v73/e062329

Muschik, C. A., I. de Vega, D. Porras, and J. I. Cirac, 2008, Phys. Rev. Lett. 100, 063601, http://link.aps. org/abstract/PRL/v100/e063601.

Neergaard-Nielsen, J. S., B. M. Nielsen, C. Hettich, K. Mølmer, and E. S. Polzik, 2006, Phys. Rev. Lett. 97, 083604, http://link.aps.org/abstract/PRL/v97/ e083604

Nielsen, M., and I. Chuang, 2000, Quantum Computation and Qauntum Information (Cambridge University Press, Cambridge).

Nilsson, M., L. Rippe, S. Kroll, R. Klieber, and D. Suter, 2004, Phys.Rev. B 70, 214116, http://link.aps.org/ abstract/PRB/v70/e214116

Novikova, I., A. V. Gorshkov, D. F. Phillips, A. S. Sørensen, M. D. Lukin, and R. L. Walsworth, 2007, Phys. Rev. Lett. 98, 243602, http://link.aps.org/abstract/PRL/ v98/e243602.

Nunn, J., I. A. Walmsley, M. G. Raymer, K. Surmacz, F. C. Waldermann, Z. Wang, and D. Jaksch, 2007, Phys. Rev. A 75, 011401(R), http://dx.doi.org/10.1103/PhysRevA. 75.011401.

Oblak, D., P. G. Petrov, C. L. G. Alzar, W. Tittel, A. K. Vershovski, J. K. Mikkelsen, J. L. Sørensen, and E. S. Polzik, 2005, Phys. Rev. A 71, 043807, http://link.aps.org/ abstract/PRA/v71/e043807

Ottaviani, C., D. Vitali, M. Artoni, F. Cataliotti, and P. Tombesi, 2003, Phys. Rev. Lett. 90, 197902, http:
//dx.doi.org/10.1103/PhysRevLett.90.197902

Ourjoumtsev, A., R. Tualle-Brouri, J. Laurat, and P. Grangier, 2006, Science 312, 83, http://www.sciencemag.org/ cgi/content/abstract/312/5770/83

Paris, M., M. Cola, N. Piovella, and R. Bonifacio, 2003, Opt. Comm. 227, 349, http://dx.doi.org/10.1016/j . optcom.2003.09.065

Petrosyan, D., and M. Fleischhauer, 2008, Phys. Rev. Lett. 100, 170501, http://link.aps.org/abstract/PRL/v100/ e170501.

Petrov, P. G., D. Oblak, C. L. G. Alzar, N. Kjærgaard, and E. S. Polzik, 2007, Phys. Rev. A 75, 033803, http://link. aps.org/abstract/PRA/v75/e033803

Pfennigbauer, M., M. Aspelmeyer, W. R. Leeb, G. Baister, T. Dreischer, T. Jennewein, G. Neckamm, J. M. Perdigues, H. Weinfurter, and A. Zeilinger, 2005, Journal Of Optical Networking 4, 549.

Phillips, D. F., A. Fleischhauer, A. Mair, R. L. Walsworth, and M. D. Lukin, 2001, Phys. Rev. Lett. 86, 783, http: //dx.doi.org/10.1103/PhysRevLett.86.783

Pittman, T. B., and J. D. Franson, 2002, Phys. Rev. A 66, 062302, http://dx.doi.org/10.1103/PhysRevA.66. 062302

Poizat, J. P., J. F. Roch, and P. Grangier, 1994, Annales De Physique 19, 265.

Polzik, E., B. Julsgaard, J. Sherson, and J.L.Sørensen, 2003, Philosophical Transactions of the Royal Society of London A361, 1391, http://dx.doi.org/10.1098/rsta. 2003.1208

Pu, H., and P. Meystre, 2000, Phys. Rev. Lett. 85, 3987, http://link.aps.org/abstract/PRL/v85/p3987

Rabl, P., D. DeMille, J. M. Doyle, M. D. Lukin, R. J. Schoelkopf, and P. Zoller, 2006, Phys. Rev. Lett. 97, 033003, http://link.aps.org/abstract/PRL/v97/ e033003.

Ralph, T. C., and P. K. Lam, 1998, Phys. Rev. Lett. 81, 5668, http://link.aps.org/abstract/PRL/v81/p5668.

Raymer, M. G., and J. Mostowski, 1981, Phys. Rev. A 24, 1980, http://dx.doi.org/10.1103/PhysRevA.24.1980

Riebe, M., H. Haffner, C. Roos, W. Hansel, J. Benhelm, G. Lancaster, T. Korber, C. Becher, F. Schmidt-Kaler, D. James, and R. B. R, 2004, Nature 429, 724, http: //dx.doi.org/10.1038/nature02570

Sangouard, N., C. Simon, M. Afzelius, and N. Gisin, 2007, Phys. Rev. A 75, 032327, http://link.aps.org/ abstract/PRA/v75/e032327.

Sangouard, N., C. Simon, B. Zhao, Y.-A. Chen, H. de Riedmatten, J.-W. Pan, and N. Gisin, 2008, eprint arXiv:0802.1475v1, http://arxiv.org/abs/0802.1475.

Schneider, S., A. Kasper, C. vom Hagen, M. Bartenstein, B. Engeser, T. Schumm, I. Bar-Joseph, R. Folman, L. Feenstra, and J. Schmiedmayer, 2003, Phys. Rev. A 67, 023612, http://dx.doi.org/10.1103/PhysRevA.67.023612

Sherson, J., J. Fiurăśek, K. Mølmer, A. Sørensen, and E. Polzik, 2006a, Phys. Rev. A 74, 011802, http://link. aps.org/abstract/PRA/v74/e011802

Sherson, J., B. Julsgaard, and E. S. Polzik, 2006b, Adv. At. Mol. Opt. Phys. 54, 81, http://dx.doi.org/10.1016/ S1049-250X (06) 54002-9.

Sherson, J. F., H. Krauter, R. K. Olsson, B. Julsgaard, K. Hammerer, I. Cirac, and E. S. Polzik, 2006c, Nature 443, 557, http://dx.doi.org/10.1038/nature05136

Shuker, M., O. Firstenberg, R. Pugatch, A. Ron, and N. Davidson, 2007, eprint arXiv:0707.3707v1, http:// 
arxiv.org/abs/0707.3707

Simon, C., H. de Riedmatten, M. Afzelius, N. Sangouard, H. Zbinden, and N. Gisin, 2007a, Phys. Rev. Lett. 98, 190503, http://link.aps.org/abstract/PRL/ v98/e190503.

Simon, J., H. Tanji, S. Ghosh, and V. Vuletic, 2007b, Nat. Phys. 3, 765, http://dx.doi.org/10.1038/nphys726.

Simon, R., 2000, Phys. Rev. Lett. 84, 2726, http://dx.doi. org/10.1103/PhysRevLett.84.2726.

Sørensen, A., L. M. Duan, J. I. Cirac, and P. Zoller, 2001, Nature 409, 63, http://dx.doi.org/10.1038/35051038.

Sørensen, A., and K. Mølmer, 1999, Phys. Rev. Lett. 83, 2274, http://link.aps.org/abstract/PRL/v83/p2274

Sørensen, A. S., and K. Mølmer, 2001, Phys. Rev. Lett. 86, 4431, http://link.aps.org/abstract/PRL/v86/p4431

Sørensen, M. W., and A. S. Sørensen, 2008, Phys. Rev. A 77,013826, http://link.aps.org/abstract/PRA/v77/ e013826

Staudt, M. U., S. R. Hastings-Simon, M. Nilsson, M. Afzelius, V. Scarani, R. Ricken, H. Suche, W. Sohler, W. Tittel, and N. Gisin, 2007, Phys. Rev. Lett. 98, 113601, http: //link.aps.org/abstract/PRL/v98/e113601.

Surmacz, K., J. Nunn, F. C. Waldermann, K. C. Lee, Z. Wang, I. A. Walmsley, and D. Jaksch, 2007, eprint arXiv:0710.5033v1, http://arxiv.org/abs/0710.5033.

Surmacz, K., J. Nunn, F. C. Waldermann, Z. Wang, I. A. Walmsley, and D. Jaksch, 2006, Phys. Rev. A 74, http: //dx.doi.org/10.1103/PhysRevA.74.050302

Takeuchi, M., S. Ichihara, T. Takano, M. Kumakura, T. Yabuzaki, and Y. Takahashi, 2005, Phys. Rev. Lett. 94, 023003, http://link.aps.org/abstract/PRL/v94/ e023003.

Thompson, J. K., J. Simon, H. Loh, and V. Vuletic, 2006, Science 313, 74, http://www . sciencemag . org/cgi/content/ abstract/313/5783/74.

Thomsen, L. K., S. Mancini, and H. M. Wiseman, 2002a, J. Phys. B: At. Mol. Opt. Phys. 35, 4937, http://dx.doi. org/10.1088/0953-4075/35/23/316

Thomsen, L. K., S. Mancini, and H. M. Wiseman, 2002b, Phys. Rev. A 65, 061801, http://link.aps.org/ abstract/PRA/v65/e061801

Tóth, G., C. Knapp, O. Gühne, and H. J. Briegel, 2007, Phys. Rev. Lett. 99, 250405, http://link.aps.org/abstract/ PRL/v99/e250405.

Vaidman, L., 1994, Phys. Rev. A 49, 1473, http://link. aps.org/abstract/PRA/v49/p1473

Vasilyev, D. V., I. V. Sokolov, and E. S. Polzik, 2008, Phys.
Rev. A 77, 020302, http://link.aps.org/abstract/PRA/ v77/e020302.

Vudyasetu, P. K., R. M. Camacho, and J. C. Howell, 2008, Phys. Rev. Lett. 100, 123903, http://link.aps.org/ abstract/PRL/v100/e123903.

Waks, E., E. Diamanti, and Y. Yamamoto, 2006, New Journal of Physics 8, 4, http://dx.doi.org/10.1088/1367-2630/ 8/1/004

van der Wal, C. H., M. D. Eisaman, A. Andre, R. L. Walsworth, D. F. Phillips, A. S. Zibrov, and M. D. Lukin, 2003, Science 301, 196, http://www. sciencemag.org/cgi/ content/abstract/301/5630/196

Wang, X. G., and B. C. Sanders, 2003, Phys. Rev. A 68, 033821, http://link.aps.org/doi/10.1103/PhysRevA. 68.033821

Wang, Z.-B., K.-P. Marzlin, and B. C. Sanders, 2006, Phys. Rev. Lett. 97, 063901, http://link.aps.org/abstract/ PRL/v97/e063901.

Windpassinger, P. J., D. Oblak, P. G. Petrov, M. Kubasik, M. Saffman, C. L. G. Alzar, J. Appel, J. H. Müller, N. Kjærgaard, and E. S. Polzik, 2008, Phys. Rev. Lett. 100, 103601, http://link.aps.org/abstract/PRL/v100/ e103601.

Wineland, D. J., J. J. Bollinger, W. M. Itano, and D. J. Heinzen, 1994, Phys. Rev. A 50, 67, http://link.aps. org/doi/10.1103/PhysRevA.50.67

Wineland, D. J., J. J. Bollinger, W. M. Itano, F. L. Moore, and D. J. Heinzen, 1992, Phys. Rev. A 46, R6797, http: //link.aps.org/abstract/PRA/v46/pR6797

Wolf, M. M., G. Giedke, O. Kruger, R. F. Werner, and J. I. Cirac, 2004, Phys. Rev. A 69, 052320, http://dx.doi. org/10.1103/PhysRevA.69.052320

Wolf, M. M., D. Perez-Garcia, and G. Giedke, 2007, Phys. Rev. Lett. 98, 130501, http://link.aps.org/abstract/ PRL/v98/e130501.

Wootters, W. K., 1998, Phys. Rev. Lett. 80, 2245, http: //dx.doi.org/10.1103/PhysRevLett.80.2245.

Yuan, Z.-S., Y.-A. Chen, B. Zhao, S. Chen, J. Schmiedmayer, and J.-W. Pan, 2008, eprint arXiv:0803.1810v1, http:// arxiv.org/abs/0803.1810.

Zare, R., 1988, Angular Momentum (John Wiley\&Sons, New York).

Zoller, P., T. Beth, D. Binosi, R. Blatt, H. Briegel, D. Bruss, T. Calarco, J. I. Cirac, D. Deutsch, J. Eisert, A. Ekert, C. Fabre, et al., 2005, Eur. Phys. J. 36, 203, http://dx. doi.org/10.1140/epjd/e2005-00251-1. 